\title{
HETEROGENEOUS REACTIONS OF EPOXIDES IN ACIDIC MEDIA
}

\author{
A Thesis \\ by \\ VINITA LAL \\ Submitted to the Office of Graduate Studies of \\ Texas A\&M University \\ in partial fulfillment of the requirements for the degree of \\ MASTER OF SCIENCE
}

December 2011

Major Subject: Atmospheric Sciences 
Heterogeneous Reactions of Epoxides in Acidic Media

Copyright 2011 Vinita Lal 


\title{
HETEROGENEOUS REACTIONS OF EPOXIDES IN ACIDIC MEDIA
}

\author{
A Thesis \\ by \\ VINITA LAL \\ Submitted to the Office of Graduate Studies of \\ Texas A\&M University \\ in partial fulfillment of the requirements for the degree of \\ MASTER OF SCIENCE
}

\begin{abstract}
Approved by:
Chair of Committee, Renyi Zhang

Committee Members, Sarah Brooks

Don Collins

Qi Ying

Head of Department, Kenneth Bowman
\end{abstract}

December 2011

Major Subject: Atmospheric Sciences 


\begin{abstract}
Heterogeneous Reactions of Epoxides in Acidic Media. (December 2011)

Vinita Lal, B.Sc.; M.Sc., Banaras Hindu University, India

Chair of Advisory Committee: Dr. Renyi Zhang
\end{abstract}

Epoxides have been recently identified as one of the intermediate species in the gas phase oxidation of alkenes. This study investigates the reaction of isoprene oxide and $\alpha$-pinene oxide with sulfuric acid to identify the potential of epoxides as important secondary organic aerosol (SOA) precursors. The reaction was explored using different methods to understand the factors governing the reaction rate and the types of products formed under different conditions. Uptake experiments of epoxides on sulfuric acid using Ion drift-Chemical Ionization Mass Spectrometry (ID-CIMS) showed an irreversible uptake of epoxides at room temperature resulting in the formation of less volatile products like diols, organosulfates and acetals. However, at lower temperatures, dehydration of diols and some rearrangement was the preferred reaction pathway resulting in the formation of higher volatility compounds like hydroxy-alkenes and aldehydes. The uptake coefficients of isoprene oxide and $\alpha$-pinene oxide at room temperature using $96 \%$ wt acid were found to be $4 \times 10^{-2}$ and $0.8 \times 10^{-2}$, respectively. Spectroscopic study using Attenuated total reflection-Fourier transform infrared technique (ATR-FTIR) revealed that for both the epoxides, diols were the major identifiable products at low acid concentrations. At higher acid concentrations, acetal formation was observed in case of isoprene oxide, while organosulfate formation was seen for $\alpha$-pinene 
oxide. No products were identified under neutral conditions due to slow reaction. Bulk studies using Nuclear Magnetic Resonance (NMR) spectroscopy conducted at low acid concentrations showed the presence of 1,2- and 1,4-diols as the major products for isoprene oxide, similar to the results from the ATR-FTIR experiments. Additionally, aldehyde formation was also observed. For $\alpha$-pinene oxide, organosulfate formation was observed in all NMR experiments, unlike ATR-FTIR results, where organosulfate formation was observed only at high acid concentrations. These observations can be attributed to the kinetic isotope effect (KIE) due to use of $\mathrm{D}_{2} \mathrm{SO}_{4} / \mathrm{D}_{2} \mathrm{O}$ in NMR experiments rather than $\mathrm{H}_{2} \mathrm{SO}_{4} / \mathrm{H}_{2} \mathrm{O}$. The percent yield of organosulfate products was proportional to the amount of available acidic sulfate. The results from this study suggest that acid hydrolysis of epoxides can result in the formation of a wide range of products under different conditions, that can contribute to SOA growth. It proves that epoxides can be efficient SOA precursors for ambient conditions prevailing in an urban atmosphere. 


\section{DEDICATION}

I would like to dedicate this work to my parents who always had faith in me and stood by my side in all the good and bad times. I owe my successes in life to them who have always inspired me and motivated me to aim high. 


\section{ACKNOWLEDGEMENTS}

I would like to thank my committee chair, Dr. Zhang, and my committee members, Dr. Brooks, Dr. Collins and Dr. Ying, for their guidance and support throughout the course of this research.

My sincere thanks goes to Dr. Khalizov for his consistent support and guidance, which made this project possible. I would also like to thank Dr. Wang and Dr. Zheng for being so encouraging at every step, and all other members of my research group for extending their unconditional help throughout my research experience.

I would like to thank Dr. Brian Connell and his student Dr. Maria Galvan from the Department of Chemistry for helping me to carry out the NMR experiments and guiding me at every step on all the matters related to the NMR studies.

My special thanks goes to Dr. Brooks and her research group for making the Raman facility available for my research purpose and other laboratory help whenever required.

I also want to extend my gratitude to the SEM facility at the university for assisting me with some parts of my project.

My heartfelt thanks goes to all my friends and colleagues and the department faculty and staff for making my time at Texas A\&M University a great experience.

Finally, thanks to my parents and in-laws for their encouragement and to my husband for his patience and love. 
ATR-FTIR

BVOC

CD

ID-CIMS

IVOC

KIE

$\mathrm{m} / \mathrm{z}$

MF

MW

NMR

SOA

SVOC

VOC
Attenuated total reflectance-Fourier Transform Infrared Spectroscopy

Biogenic volatile organic compounds

Corona discharge

Ion drift-Chemical Ionization Mass Spectrometry

Intermediate-volatile organic compounds

Kinetic Isotopic effect

Mass to charge ratio

Molecular formula

Molecular weight

Nuclear Magnetic Resonance

Secondary organic aerosol

Semi-volatile organic compounds

Volatile organic compounds 


\section{TABLE OF CONTENTS}

Page

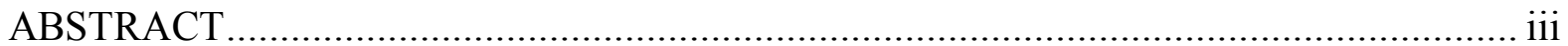

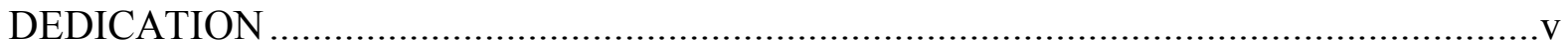

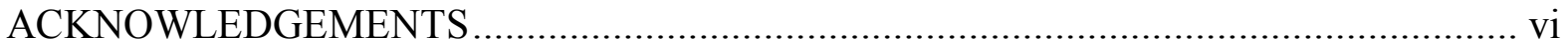

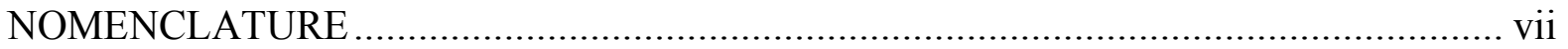

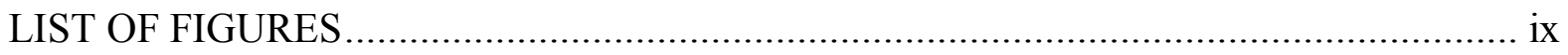

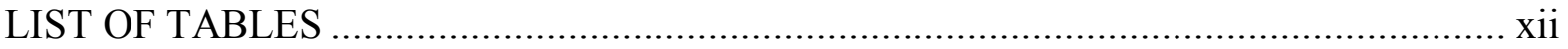

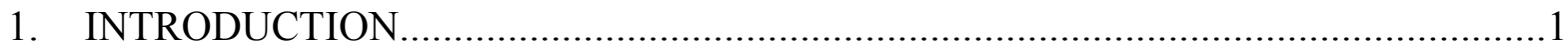

2. EXPERIMENTAL DETAILS .......................................................................

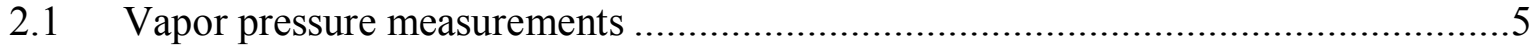

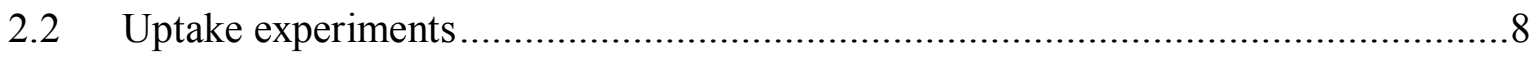

2.3 ATR-FTIR measurements .................................................................. 15

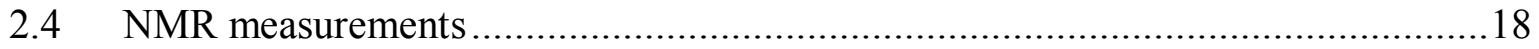

2.5 Optical microscope measurements..........................................................20

3. RESULTS AND DISCUSSION .................................................................... 21

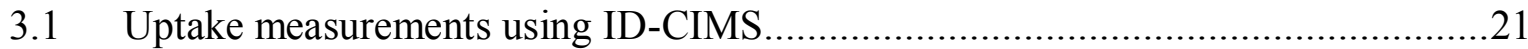

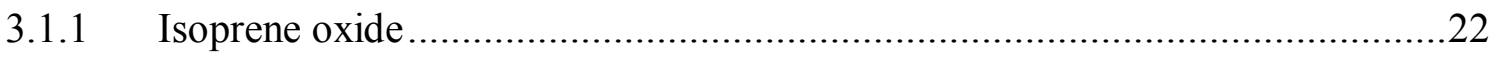

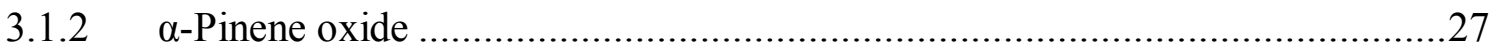

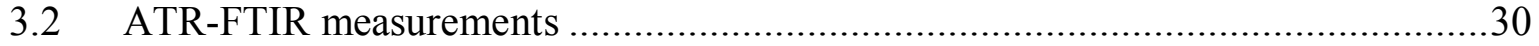

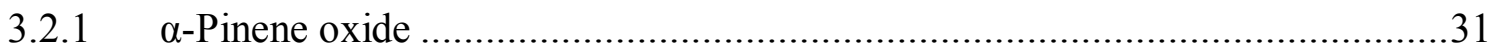

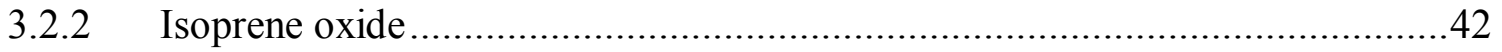

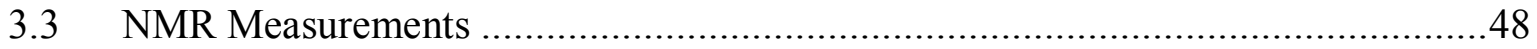

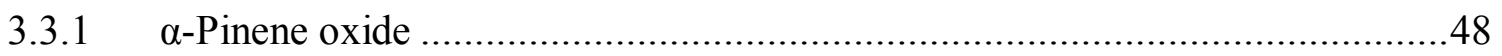

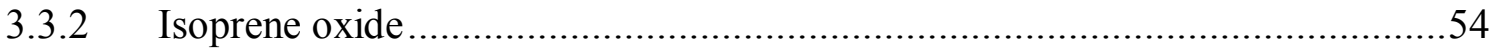

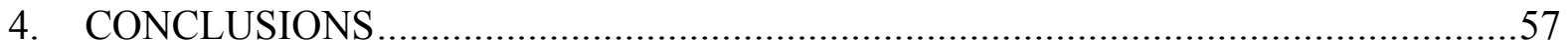

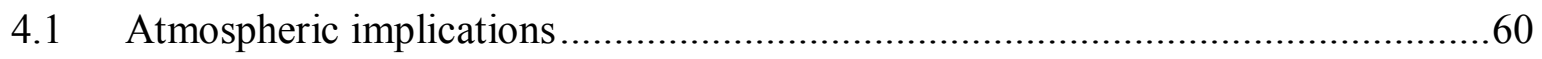

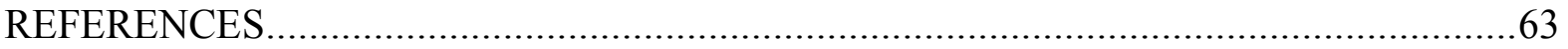

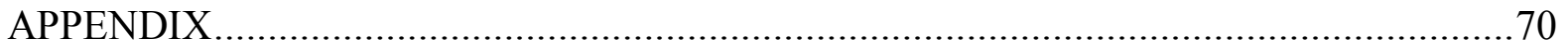

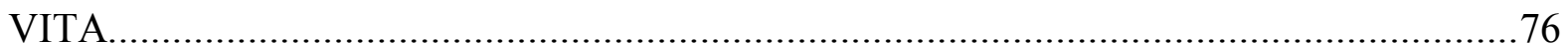




\section{LIST OF FIGURES}

Page

Figure 1: ln $\mathrm{P}$ vs $1 / \mathrm{T}$ plot for isoprene oxide. Vapor pressure at $-40^{\circ} \mathrm{C}(233 \mathrm{~K})$ calculated from this plot was $\sim 1.3$ torr.

Figure 2: Experiment set-up of ID-CIMS used for uptake measurements. .10

Figure 3: Schematic representation of ATR-FTIR set-up. 16

Figure 4: Temporal profiles of m/z 116 under different conditions using $\mathrm{O}_{2}{ }^{-}$as reagent ion (a) isoprene oxide cluster with $\mathrm{O}_{2}^{-} \mathrm{m} / \mathrm{z} 116$ decreases on exposure to $96 \% \mathrm{wt}$ acid with $\mathrm{T}=298 \mathrm{~K}, \mathrm{P}=1.68$ torr, $\mathrm{u}=664 \mathrm{~cm} \mathrm{~s}^{-1}$. (b) Hydroxy alkene/aldehyde cluster with $\mathrm{O}_{2}^{-} \mathrm{m} / \mathrm{z}$ at $283 \mathrm{~K}$

Figure 5: Response of ions m/z 43 and 85 on successive addition of acid to epoxide-water mixture.

Figure 6: (a) Acid catalyzed products for isoprene oxide. (b) Acid-catalyzed isomerization products for $\alpha$-pinene oxide with same $\mathrm{m} / \mathrm{z}$ as the epoxide.

Figure 7: ATR-FTIR spectra of $\alpha$-pinene oxide reaction with sulfuric acid at (a) $84 \% \mathrm{RH}$ (b) $42 \% \mathrm{RH}$ (c) $9 \% \mathrm{RH}$. Total time for the reactions was $70 \mathrm{~min}$ in each case. .....32

Figure 8: (a) ATR-FTIR spectra in the fingerprint region for the product of acid-epoxide reaction (for $\alpha$-pinene oxide) under different humidity conditions. (b) Integrated $\mathrm{C}$-H absorbance (3000-2850 $\mathrm{cm}^{-1}$ ) over time for $\alpha$-pinene oxide at different RH...34

Figure 9: Optical microscopic images of sulfuric acid reaction with $\alpha$-pinene oxide (a) at $9 \% \mathrm{RH}$ after $30 \mathrm{~min}$ (b) at $84 \% \mathrm{RH}$ after $30 \mathrm{~min}$. Other images include (c) sulfuric acid exposed to water vapor only at $84 \%$ RH after 30 min (d) sulfuric acid exposed to epoxide at $80 \%$ RH after 5 min (e) ammonium bisulfate exposed to epoxide after $30 \mathrm{~min}$.

Figure 10: Formation of organosulfate by $\alpha$-pinene oxide under acidic conditions.

Figure 11: (a) Integrated absorbance of C-H (3000-2850 $\left.\mathrm{cm}^{-1}\right)$ peak of $\alpha$-pinene oxide reaction with sulfuric acid over time for different flow rates. (b) Integrated absorbance of $\mathrm{C}-\mathrm{H}\left(3000-2850 \mathrm{~cm}^{-1}\right)$ peak of $\alpha$-pinene oxide reaction with sulfuric acid after $30 \mathrm{~min}$ at different flow rates. 
Figure 12: Integrated absorbance for $\mathrm{C}-\mathrm{H}\left(3000-2850 \mathrm{~cm}^{-1}\right)$ peak of $\alpha$-pinene oxide reaction with $\mathrm{H}_{2} \mathrm{SO}_{4}$ after 30 min for different epoxide concentrations at two $\mathrm{RH}$ conditions.

Figure 13: ATR-FTIR spectra of reaction of $\alpha$-pinene oxide with deliquesced ammonium bisulfate.

Figure 14: ATR-FTIR spectra of isoprene oxide reaction with sulfuric acid at (a) $80 \% \mathrm{RH}$ (b) $41 \% \mathrm{RH}$ (c) $13 \% \mathrm{RH}$. Total time for the reactions was $30 \mathrm{~min}$ for the $\mathrm{RH}<=$ $41 \%$ and $45 \mathrm{~min}$ for low $\mathrm{RH}$.

Figure 15: (a) IR spectra in the fingerprint region for the product of acid-epoxide reaction (for isoprene epoxide) under different humidity conditions (b) Integrated $\mathrm{C}-\mathrm{H}$ absorbance over time for isoprene oxide at different $\mathrm{RH}$.

Figure 16: Integrated absorbance for C-H (3000-2850 $\left.\mathrm{cm}^{-1}\right)$ peak of isoprene oxide reaction with $\mathrm{H}_{2} \mathrm{SO}_{4}$ after 30 min for different epoxide concentrations at $13 \%$ RH.

Figure 17: ${ }^{1} \mathrm{H}$ NMR spectrum of $\alpha$-pinene oxide reaction with $0.7 \%$ wt $\mathrm{D}_{2} \mathrm{SO}_{4}$ at $298 \mathrm{~K}$. The chemical shifts depicted are associated with the protons attached to carbons with the respective functional groups.

Figure 18: Relative proportions of products formed in different experiments of $\alpha$-pinene oxide with deuterated sulfuric acid.

Figure 19: Sulfate ester product yield as a function of available acidic sulfate in solution. ...53

Figure 20: Relative proportions of products formed in different experiments of isoprene oxide with deuterated sulfuric acid.

Figure 21: ${ }^{1} \mathrm{H}$ NMR spectrum of isoprene oxide reaction with $5 \%$ wt $\mathrm{D}_{2} \mathrm{SO}_{4}$ at $298 \mathrm{~K}$. The chemical shifts depicted are associated with the protons attached to carbons with the respective functional groups.

Figure 22: ATR-FTIR spectra of sulfuric acid at different RH.

Figure 23: ATR-FTIR spectra at different RH for (a) Ammonium sulfate (b) Ammonium bisulfate.

Figure 24: ${ }^{1} \mathrm{H}$ NMR spectrum of $\alpha$-pinene oxide reaction with $0.1 \%$ wt $\mathrm{D}_{2} \mathrm{SO}_{4} / 5 \%$ wt $\mathrm{Na}_{2} \mathrm{SO}_{4}$ solution. 
Figure 26: ${ }^{1} \mathrm{H}$ NMR spectrum of $\alpha$-pinene oxide reaction with $0.02 \%$ wt $\mathrm{D}_{2} \mathrm{SO}_{4}$ solution. ...72

Figure 27: ${ }^{1} \mathrm{H}$ NMR spectrum of $\alpha$-pinene oxide reaction with $0.3 \%$ wt $\mathrm{D}_{2} \mathrm{SO}_{4}$ solution. .....72

Figure 28: ${ }^{1} \mathrm{H}$ NMR spectrum of $\alpha$-pinene oxide reaction with $0.7 \%$ wt $\mathrm{D}_{2} \mathrm{SO}_{4}$ solution. .....73

Figure 29: ${ }^{1} \mathrm{H}$ NMR spectrum of $\alpha$-pinene oxide reaction with $5 \% \mathrm{D}_{2} \mathrm{SO}_{4}$ with a higher

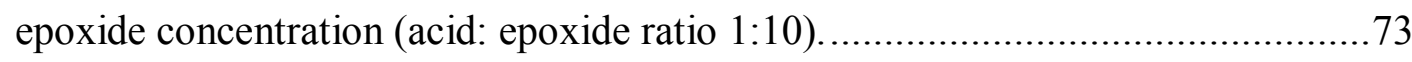

Figure 30: ${ }^{1} \mathrm{H}$ NMR spectrum of overnight experiment of $\alpha$-pinene oxide reaction with 5\%

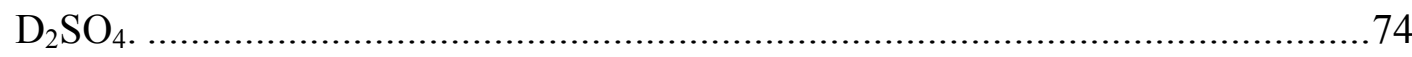

Figure 31: ${ }^{1} \mathrm{H}$ NMR spectrum of isoprene oxide reaction with $5 \%$ wt $\mathrm{D}_{2} \mathrm{SO}_{4}$ solution..........74

Figure 32: ${ }^{1} \mathrm{H}$ NMR spectrum of isoprene oxide reaction with $0.7 \%$ wt $\mathrm{D}_{2} \mathrm{SO}_{4}$ solution.......75

Figure 33: ${ }^{1} \mathrm{H}$ NMR spectrum of isoprene oxide reaction with $5 \%$ wt $\mathrm{D}_{2} \mathrm{SO}_{4}$ solution with higher epoxide concentration (acid: epoxide ratio $1: 10$ )................................. 75 


\section{LIST OF TABLES}

Page

Table 1: Epoxides used in the study ................................................................

Table 2: Antoine equation constants for isoprene oxide vapor pressure calculation ..............6

Table 3: Reagent and product ions monitored using ID-CIMS ................................... 12

Table 4: Experimental details for the ATR-FTIR experiments .....................................17

Table 5: Experimental details for the NMR experiments .............................................. 19

Table 6: ATR-FTIR absorption frequencies assigned to the functional groups formed by

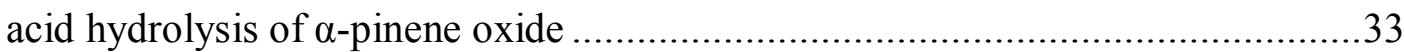

Table 7: ATR-FTIR absorption frequencies assigned to the functional groups formed by

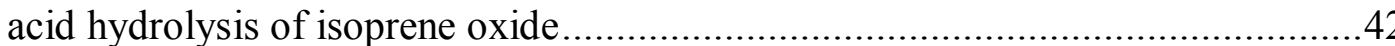




\section{INTRODUCTION}

Volatile organic compounds (VOCs) are chemically transformed in the atmosphere to produce oxidation products which are usually low vapor pressure compounds [Kroll and Seinfeld, 2008; Surratt et al., 2006]. These oxidation products lead to the formation of secondary organic aerosols (SOA). The SOA constitute a major part of the tropospheric aerosol load, contributed both by biogenic as well as anthropogenic sources. Aerosols including SOAs have been of great interest since the past couple of decades due to their various effects on visibility, climate and human health [Metzger et al., 2010; Lin Wang et al., 2010]. The poor understanding of the processes involved in SOA formation leads to uncertain estimation of SOA yields from various sources [Lin Wang et al., 2010]. Several processes have been accounted so far leading to SOA formation and growth from organic precursors, such as condensation of the low-volatility organics on pre-existing particles, gasto-particle partitioning of the semi-volatile (SVOC) and intermediate volatility (IVOC) organics, and by heterogeneous reactions catalyzed by acidic surfaces [Hallquist et al., 2009; Lin Wang et al., 2010]. Photolysis and gas phase oxidation of VOCs by $\mathrm{OH}, \mathrm{O}_{3}, \mathrm{NO}_{3}$ leads to formation of different oxidized species, some contributing to new particle formation and growth more efficiently than others. The complexity and uncertainties arise when the first

generation of oxidation products participate in other reactions and undergo transformation to form a range of oxidized species.

This thesis follows the style of Journal of Geophysical Research. 
These second generation products may be less volatile and enhance SOA growth, or may be more volatile, thus escaping back into the gas phase. Understanding the properties of these oxidized forms and reactions they participate in thus becomes very important in order to improve our knowledge about SOA formation and composition.

SOA precursors are both of biogenic and anthropogenic origin, but the biogenic VOCs (BVOCs) outnumber the anthropogenic SOA precursors by a wide margin [Hallquist et al., 2009; Kanakidou et al., 2005]. Isoprene and monoterpenes constitute the largest fraction of biogenic organic species in the atmosphere contributing to SOA formation. Isoprene has an annual emission budget of $\sim 600 \mathrm{Tg} / \mathrm{yr}$ [Guenther et al., 2006; Guenther et al., 1995], and has been extensively studied as a successful SOA precursor. The global estimate of monoterpenes as measured by various models is varied over a wide range and is around 127 Tg/yr [Arneth et al., 2008; Guenther et al., 1995]. They have been identified as important SOA precursors [Kroll and Seinfeld, 2008] through their photooxidation and ozonolysis reactions. [Yu et al., 2008]. Among the monoterpenes, $\alpha$-pinene is one of the most widely studied BVOC [Guenther et al., 1995] and due to its reactivity with atmospheric oxidants, has proved to be a significant contributor to SOA formation [Yu et al., 2008]. Generally, highly volatile species are not considered as good SOA precursors, so the SOA yields from isoprene as such are lower than that from monoterpenes or sesquiterpenes. However, the huge global budget of isoprene and the large number of low volatile oxidation products of isoprene participating in a series of reactions might lead to a significant contribution to SOA [Carlton et al., 2009]. Photooxidation and acid catalyzed heterogeneous reactions of isoprene have been shown to produce small amounts of SOA and lead to several first and second generation oxidation products that eventually form SOA by further oxidation, condensation 
and aqueous phase reactions [Carlton et al., 2009]. Recent laboratory studies have shown significant SOA production from acid catalyzed reactions of isoprene and $\alpha$-pinene oxidation products [Iinuma et al., 2009; Surratt et al., 2007; Surratt et al., 2008]. Acid catalyzed reactions result in formation of low volatile species including organic sulfates, nitrates and oligomers. Isoprene derived organosulfates have also been observed in urban atmospheres [Hatch et al., 2011]. The oxidation products of these VOCs such as alcohols, carbonyls, acids which have lower vapor pressures than their parent VOC further participate in gas and aqueous phase reactions leading to more SOA formation. Several studies have been done on the SOA forming efficiency of these oxidation products [Iinuma et al., 2007; Levitt et al., 2006; Metzger et al., 2010; Minerath et al., 2008; Lin Wang et al., 2010; Renyi Zhang et al., 2004; Zhao et al., 2006]. Very recently, epoxide has been identified as an intermediate product in the photooxidation of isoprene by $\mathrm{OH}$ in low $\mathrm{NO}_{\mathrm{x}}$ conditions [Paulot et al., 2009; Surratt et al., 2010]. Epoxides have also been found to be produced in high yields in gas phase oxidation of alkenes by $\mathrm{O}\left({ }^{3} \mathrm{P}\right)$ [Alvarado et al., 1998; Atkinson et al., 1994a; Atkinson et al., 1994b] and in minor quantities by ozone [Atkinson et al., 1994a; Atkinson et al., 1994b] and $\mathrm{NO}_{3}$ [Benter et al., 1994; Berndt and Boge, 1997a; b; Skov et al., 1994]. Epoxides are compounds with very high reactivity, and their reaction with acid leading to formation of low volatile species have been shown in recent studies [Darer et al., 2011; Eddingsaas et al., 2010; Iinuma et al., 2009; Minerath and Elrod, 2009; Minerath et al., 2009]. In one of these studies, it has also been shown that organosulfate formation is kinetically favorable for epoxides compared to other oxidized products like alcohols [Minerath and Elrod, 2009]. Organosulfates are an important class of SOA. They form in the presence of partially neutralized sulfuric acid aerosol, one which is found in abundance in the 
atmosphere. Sulfuric acid acts as a catalyst and provides a surface for heterogeneous reactions, a class of reactions that has opened a new insight to look at the chemistry involved in the SOA formation. Most work till now focused on SOA formation by gas phase oxidation and condensation of the low volatility organic vapors. But these processes alone cannot account for the increase in mass and growth of SOAs. Heterogeneous chemistry including reactions catalyzed by acid, involving hydration and polymerization, hemiacetal formation and accretion reactions have been studied recently which can explain enhanced growth of SOA under certain atmospheric conditions [Jang et al., 2002; Zhao et al., 2006].

In the current study, the reactions of two epoxides, isoprene oxide and $\alpha$-pinene oxide with sulfuric acid have been explored. Sulfuric acid is a major nucleating species and important inorganic precursor in SOA formation [Doyle, 1961]. Reactions of these epoxides with a range of acidities has been studied to identify the types of products formed and hence, obtain a better understanding of the mechanisms by which these epoxides react under different conditions. The purpose of this study is to find out the role of epoxides as important SOA precursors. 


\section{EXPERIMENTAL DETAILS}

\subsection{Vapor pressure measurements}

The details of the epoxides used in this study are provided in Table 1. Both isoprene oxide (95\%) and $\alpha$-pinene oxide (97\%) were purchased from Sigma-Aldrich. The vapor pressures of the epoxides at $298 \mathrm{~K}$ as calculated using the ACD Labs software version V11.02 (1994$2011 \mathrm{ACD} / \mathrm{Labs})[A C D, 1994-2011]$ were found to be 102 torr and 0.82 torr for isoprene oxide and $\alpha$-pinene oxide, respectively. Since the vapor pressure of isoprene oxide was very high at $298 \mathrm{~K}$, vapor pressure measurements were made at other temperatures in order to get a value similar to that of $\alpha$-pinene oxide at $298 \mathrm{~K}$. Vapor pressure measurements for isoprene oxide were made at 3 different temperatures using a vacuum manifold. A small volume of epoxide was placed in a bubbler and connected to the manifold. The manifold was connected to a pump to create vacuum. The initial vacuum pressure was noted. The epoxide in the bubbler was cooled using liquid nitrogen and then subject to vacuum to remove any other volatile impurities. The bubbler was then disconnected from the manifold. It was then kept in the cooling bath required to obtain a desired temperature and allowed to saturate with epoxide vapors at that particular temperature. The bubbler containing the epoxide was reconnected to the vacuum manifold and the epoxide vapors from the bubbler were allowed to move into the manifold. At this step, the knob to the vacuum pump was closed. Observation was made till the epoxide in the bubbler started to boil. The boiling stopped after the saturation vapor pressure for the epoxide was reached. This was indicated by a constant pressure reading obtained on the pressure gauge. The pressure at this stage was noted and was considered as the saturation vapor pressure of the epoxide at that temperature. 
Table 1: Epoxides used in the study

\begin{tabular}{|l|l|l|l|}
\hline Epoxide & MF & MW & Structure \\
Isoprene oxide & $\mathrm{C}_{5} \mathrm{H}_{8} \mathrm{O}$ & 84 & \\
oxirane) & & &
\end{tabular}

Figure 1 shows the relation between the temperatures of the cooling baths and saturation vapor pressure of isoprene epoxide at the respective temperatures. A linear fit in $\ln \mathrm{P}$ vs $1 / \mathrm{T}$ coordinates was applied for the data, using the measured values of pressure and temperature. The calculated values were obtained from the modified Antoine equation:

$$
\ln (P)=A-\frac{B}{T}
$$

where $\mathrm{A}$ and $\mathrm{B}$ are constants. The values of $\mathrm{A}$ and $\mathrm{B}$ are shown in Table 2.

Table 2: Antoine equation constants for isoprene oxide vapor pressure calculation

\begin{tabular}{ll}
\hline Constants & Values \\
\hline $\mathbf{A}$ & 19.905 \\
$\mathbf{B}$ & -4592.3 \\
\hline
\end{tabular}




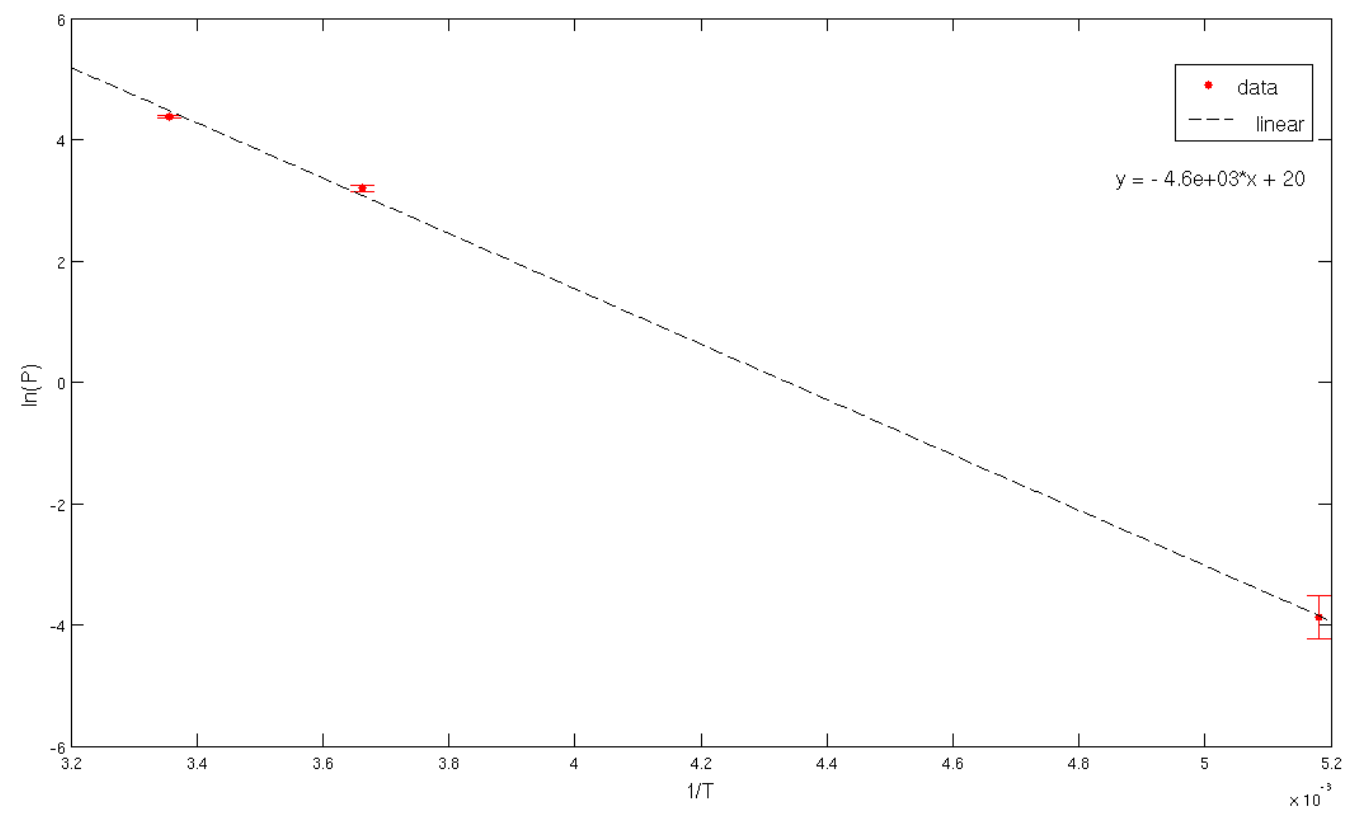

Figure 1: In $\mathrm{P}$ vs 1/T plot for isoprene oxide. Vapor pressure at $-40^{\circ} \mathrm{C}(233 \mathrm{~K})$ calculated from this plot was $\sim 1.3$ torr. 


\subsection{Uptake experiments}

The uptake experiments were carried out using the low pressure laminar flow reactor coupled to the ion drift-chemical ionization mass spectrometry (ID-CIMS), similar to other studies [Levitt et al., 2006; L. Wang et al., 2010; D Zhang and Zhang, 2005; Zhao et al., 2006]. A 50 $\mathrm{cm}$ long Pyrex flow tube with an inner radius of $1.25 \mathrm{~cm}$ was connected to the drift tube as shown in Figure 2 [L. Wang et al., 2010]. A glass trough, $20 \mathrm{~cm}$ in length and $0.7 \mathrm{~cm}$ in depth, containing sulfuric acid was placed inside the flow tube. A movable glass injector was introduced into the flow tube right above the trough containing acid. Epoxide was passed through this injector. The pressure inside the flow tube was maintained at around 1.5 torr. The temperature of the flow tube was controlled by passing SYLTHERM fluid through its outer jacket using Neslab UT-80 circulator. All carrier flows were monitored using calibrated electronic flow meters (Millipore Tylan 260 Series).

A 2-L glass bulb containing epoxide vapor (100 ppm) was used for introducing epoxide into the flow tube through the movable injector. The epoxide bulb was prepared by a multi-step dilution process. For isoprene oxide, the epoxide in a bubbler was cooled using liquid nitrogen and subjected to vacuum to remove any dissolved gases, keeping the epoxide frozen. Then the vacuum line was closed and the epoxide was brought to room temperature. This step was repeated 3-4 times to assure the purity of epoxide in the bulb. After purification, the epoxide was warmed up and vapors from the bubbler were then allowed to move into the evacuated glass bulb. It was then diluted with He in two steps to make the total concentration of epoxide inside the bulb equal to $100 \mathrm{ppm}$. This concentration was measured in terms of pressure inside the bulb with the help of a pressure gauge. For $\alpha$-pinene oxide, two approaches for bulb preparation were adopted, to obtain sufficient concentration of 
epoxide. The first approach of bulb preparation was similar to that for isoprene oxide. In the second method, about $2 \mathrm{ml}$ of epoxide was placed in an identical round flask with continuous purging of nitrogen, so as to avoid any contamination by air. Then the flask was sealed and the epoxide in the bulb was kept undisturbed overnight to let the bulb saturate with epoxide vapor. Next day, the epoxide vapor from the bulb was transferred to another bulb in known amount using a vacuum manifold, and was diluted if required to obtain a concentration of 100 ppm.

Sulfuric acid solutions of known concentrations $(65.7 \%, 75 \%$ and $85.4 \%$ wt) were prepared from $96-98 \% \mathrm{H}_{2} \mathrm{SO}_{4}$ stock solution using deionized water $(18 \mathrm{~m} \Omega \mathrm{cm})$. The glass trough was filled with acid solution and placed inside the flow tube. Before and after each experiment, the acid composition was checked by titration with standard $0.1 \mathrm{~N} \mathrm{NaOH}$ solution. Water vapor was added to the flow tube to reduce any changes in acid composition. The water vapor pressure of sulfuric acid increases with increasing temperatures and decreasing concentrations [R. Zhang et al., 1993]. So, the acid concentrations considered for this study were similar to those used in a previous study [L. Wang et al., 2010]. The temperature range for the uptake experiments using these acid concentrations was 243-283 K. Some experiments using $96 \% \mathrm{wt}$ acid at room temperature were also carried out for each epoxide. 


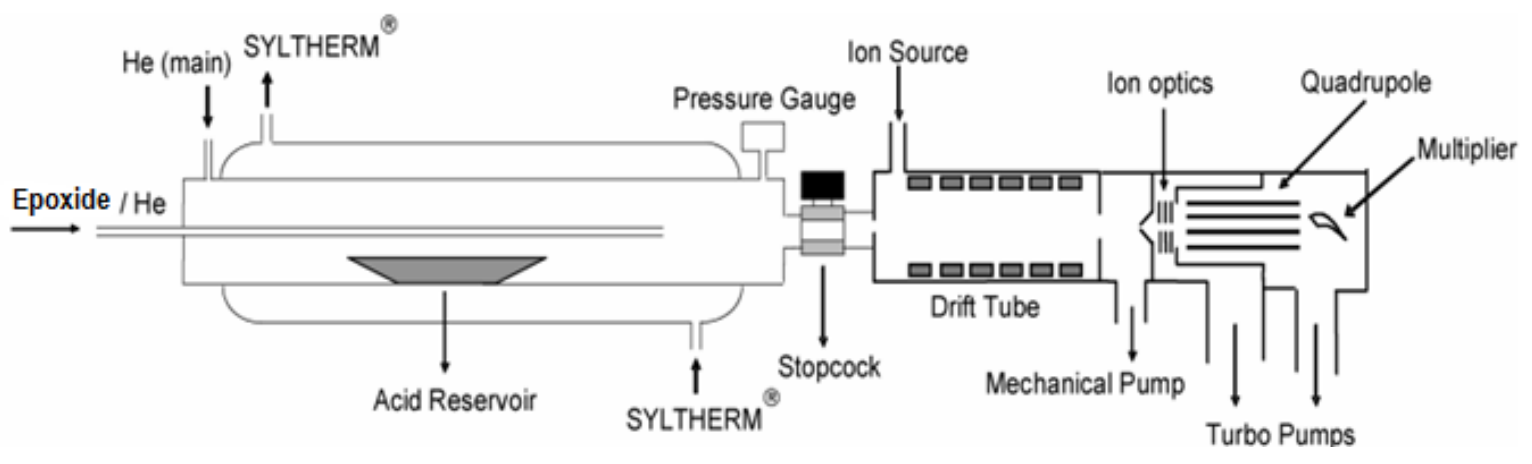

Figure 2: Experiment set-up of ID-CIMS used for uptake measurements.

The principle of ID-CIMS has been discussed previously [Fortner et al., 2004]. It involves a reaction between a charged reagent ion and a neutral analyte molecule to give a product ion. ID-CIMS is one of the modified chemical ionization methods. Chemical ionization (CI) is considered as a 'soft' technique because of smaller energy of ionization reactions. In contrast, the electron ionization (EI) technique is a 'hard' technique where ionization of the analyte molecule takes place by bombardment with electrons. This results in excessive fragmentation of the molecule. In chemical ionization mass spectrometry, the lower energy of the reagent ions leads to less fragmentation and hence better recognition of the product ions. The charged reagent ions are produced from an ion source, the one used here being a custom-made corona discharge (CD) device. Varying the drift-field voltage changes the collision energy and hence controls the fragmentation of ions. 
For proper detection of the organic ions, experiments were carried out both in positive and negative ion modes trying different reagent ions. The reaction mechanisms are as follows:

- $\mathrm{H}_{3} \mathrm{O}^{+}$

$\mathrm{H}_{3} \mathrm{O}^{+}$is one of the most widely used reagent ions for detection of VOCs [Blake et al., 2009; Fortner et al., 2009; Qiu et al., 2011; L. Wang et al., 2010]. The reaction

$$
\mathrm{H}_{3} \mathrm{O}^{+}+\mathrm{X} \rightarrow \mathrm{XH}^{+}+\mathrm{H}_{2} \mathrm{O}
$$

is called the proton-transfer reaction (PTR-MS), where $\mathrm{X}$ is the original organic molecule, or its fragment, and $\mathrm{XH}^{+}$its protonated counterpart. For isoprene oxide, the ions monitored were $\mathrm{m} / \mathrm{z} 85$ (protonated epoxide) and $\mathrm{m} / \mathrm{z} 43$ (fragment $\mathrm{C}_{2} \mathrm{H}_{3} \mathrm{O}^{+}$). For $\alpha$-pinene oxide, the ions monitored were $\mathrm{m} / \mathrm{z} 43$ and m/z 93.5 (fragments from epoxide) [Schoon et al., 2004].

\section{- $\mathrm{O}_{2}^{+}$}

Reaction with $\mathrm{O}_{2}^{+}$is called the charge transfer reaction and it occurs when the ionization energy of the reactants is smaller than the electron affinity of $\mathrm{O}_{2}{ }^{+}$[Dhooghe et al., 2008; Norman et al., 2007; Schoon et al., 2004]. It can be represented as

$$
X+O_{2}^{+} \rightarrow X^{+}+O_{2}
$$

The ions monitored in this case were $84\left(\mathrm{C}_{5} \mathrm{H}_{10} \mathrm{O}^{+}\right)$and $69\left(\mathrm{C}_{4} \mathrm{H}_{5} \mathrm{O}^{+}\right)$for isoprene oxide. $\alpha$ Pinene oxide was not monitored using this reagent ion.

- $\mathbf{O}_{2}^{-}$

The ion-molecule reaction with $\mathrm{O}_{2}{ }^{-}$proceeds by the formation of the cluster ion (association reaction) [Skalny et al., 2004; Viidanoja et al., 1998] 


$$
X+O_{2}^{-} \rightarrow X . O_{2}^{-}
$$

The ions monitored were $116\left(\mathrm{C}_{5} \mathrm{H}_{10} \mathrm{O} \mathrm{O}_{2}{ }^{-}\right)$for isoprene oxide and $184.6\left(\mathrm{C}_{10} \mathrm{H}_{16} \mathrm{O}_{2} \mathrm{O}_{2}{ }^{-}\right)$for $\alpha$ pinene oxide. The details of reagent and product ions have been summarized in Table 3 .

Table 3: Reagent and product ions monitored using ID-CIMS

\begin{tabular}{|l|l|l|l|l|}
\hline Reagent Ion & $\begin{array}{l}\text { Monitored } \\
\text { reagent Ion }(\mathbf{m} / \mathbf{z})\end{array}$ & $\begin{array}{l}\text { Monitored } \\
\text { product Ion (M) }\end{array}$ & $\begin{array}{l}\text { Isoprene } \\
\text { oxide }\end{array}$ & $\begin{array}{l}\text { a-Pinene } \\
\text { oxide }\end{array}$ \\
\hline $\mathrm{H}_{3} \mathrm{O}^{+}$ & $\mathrm{H}_{3}{ }^{18} \mathrm{O}^{+}(21)$ & $\mathrm{MH}^{+}$ & 43,85 & 43,93 \\
\hline $\mathrm{O}_{2}{ }^{+}$ & {$\left[\mathrm{O} .{ }^{18}\right]^{+}(34)$} & $\mathrm{M}^{+}$ & 84,69 & - \\
\hline $\mathrm{O}_{2}{ }^{-}$ & {$\left[\mathrm{O} .{ }^{18} \mathrm{O}\right]^{-} \cdot \mathrm{H}_{2} \mathrm{O}(52)$} & ${\mathrm{M} . \mathrm{O}_{2}}^{-}$ & 116 & 184 \\
\hline
\end{tabular}


The purpose of the uptake experiments is to calculate the uptake coefficient $\gamma$, also defined as the ratio of the reactive gas-surface collisions to the sum of all the gas-surface collisions. To calculate uptake of a gas-phase species over the planar liquid surface the following equation was used [L. Wang et al., 2010]:

$$
\gamma=\frac{4 k V}{\omega A}
$$

where $\mathrm{k}$ is the measured first order rate constant, $V$ is the volume of the flow reactor, $\mathrm{A}$ is the geometric area of the exposed acid, and $\omega$ is the mean thermal speed of the reactant molecule. The observed first order rate constant $\left(\mathrm{k}_{\mathrm{obs}}\right)$ was calculated by monitoring the loss in signal of the epoxide on exposure to acid, given by the equation below [Qiu et al., 2011; L. Wang et al., 2010]

$$
k_{o b s}=\frac{u}{L} \ln \left(\frac{I_{0}}{I_{t}}\right)
$$

where $u$ is the carrier gas flow velocity $\left(\mathrm{cm} \mathrm{s}^{-1}\right), L$ is the exposure distance (length of acid surface exposed to epoxide, $\mathrm{cm}), I_{0}$ is the initial epoxide signal before exposure to acid, and $I_{\mathrm{t}}$ is the signal at a given reaction time (with respect to the injector position).

To account for the radial gas-phase diffusion, the diffusion rate constant $k_{\text {diff }}\left(s^{-1}\right)$ was calculated using the Fuller, Schettler and Giddings correlation [Fuller et al., 1966]:

$$
k_{\text {diff }}=\frac{3.66 D_{\text {epoxide }, H e}}{r^{2}}
$$

where $\mathrm{D}_{\text {epoxide,He }}$ is the binary gas diffusivity between epoxide and $\mathrm{He}$ at a specific temperature and pressure $\left(\mathrm{cm}^{2} \mathrm{~s}^{-1}\right)$ and $\mathrm{r}$ is the radius of the flow reactor. This gas phase diffusion is calculated assuming a circular flow reactor and hence accounts for the 
uncertainty in the estimation of uptake coefficient. Here, $\mathrm{k}$ was determined according to the relation

$$
\frac{1}{k_{o b s}}=\frac{1}{k_{\text {diff }}}+\frac{1}{k}
$$

Some bulk measurements were also carried out using ID-CIMS to distinguish between interfering signals. Epoxide was mixed with $75 \%$ wt acid in 1:100 ratio at room temperature and left for an hour. The product mixture was placed in the trough and monitored using different reagent ions to observe any volatile products that may be formed. A similar experiment was performed by mixing epoxide with water in 1:200 ratio at room temperature and leaving it overnight. The product was again monitored using only $\mathrm{H}_{3} \mathrm{O}^{+}$as reagent ion. Several other experiments were performed by addition of known amounts of acid into the epoxide-water mixture and monitoring the signals for each step. All these experiments were carried out at $243 \mathrm{~K}$ with $75 \%$ wt acid concentration. 


\subsection{ATR-FTIR measurements}

ATR-FTIR studies were performed using the Nicolet Magna 560 spectrometer equipped with a liquid nitrogen cooled MCT detector. The experimental setup is shown in Figure 3 [Wang, 2011]. A ZnSe crystal mounted on a flat plate was used as a platform to study the reaction between epoxide and acid droplets. The crystal was $80 \mathrm{~mm}$ long, $10 \mathrm{~mm}$ wide and $4 \mathrm{~mm}$ thick. The instrument was purged with nitrogen at 20 psi for at least an hour before carrying out any experiment. This helps in minimizing the water and carbon dioxide peaks in sample spectra. Before collecting a sample spectrum, a background scan was taken by keeping an empty crystal on the trough. Typically, 64 scans were averaged to make a complete spectrum, with a resolution of $2 \mathrm{~cm}^{-1}$. The scan range was $4000-700 \mathrm{~cm}^{-1}$. After taking the background, sulphuric acid particles of size $\sim 40 \mathrm{~nm}$ were deposited on the crystal using a TSI 3076 Constant Output Atomizer [Wang, 2011]. The atomizer used a nitrogen flow at 30 psi pressure. The total mass of deposited sulfuric acid particles on the crystal was $\sim 30 \mu \mathrm{g}$, after exposure of $2 \mathrm{~min}$. Care was taken to maintain the thickness of the acid film to be within the penetration depth for ZnSe crystal assumed to be $\sim 2 \mu \mathrm{m}$. The size and mass of acid particles deposited on the crystal was estimated from images obtained by Scanning Electron Microscope (SEM) and optical microscope. The acid spectrum was collected and also taken in the background, so that the sample spectrum includes only the product peaks. The set-up consisted of 3 flows: a dry nitrogen flow, a moist nitrogen flow (nitrogen passing through a water bubbler), and a small nitrogen flow through the epoxide bubbler. The dry and moist flows were mixed together to achieve the desired relative humidity inside the reaction cell, and hence the acidity. The relation between acidity and $\mathrm{RH}$ was calculated using the water vapour pressure data over aqueous sulphuric acid solutions [Green and Perry, 2008]. Once 
the desired acidity was maintained, organic vapors were passed over the acid particles, and spectrum was recorded at various time intervals to observe the development of new peaks characterizing products. The initial concentration of $\alpha$-pinene oxide and isoprene oxide were measured using PTR-MS. One complete experiment included taking of the background spectrum, taking the acid spectrum and including it in the background, and then collecting the spectrum of acid-epoxide reaction. The crystal was cleaned using water and iso-propanol between experiments and a background was taken after cleaning to confirm any contamination.

All ATR-FTIR experiments were carried out at $T=296 \pm 2 \mathrm{~K}$. For $\alpha$-pinene oxide, the epoxide was kept in a bubbler connected to the nitrogen flows and kept at room temperature. For isoprene oxide, the epoxide was placed in a cooling bath containing a eutectic mixture of $31 \%$ wt. $\mathrm{CaCl}_{2}$ and water cooled using liquid $\mathrm{N}_{2}$ with a temperature of $233 \mathrm{~K}$ [Bryan and Byrne, 1970]. At this temperature, the vapor pressure of isoprene oxide was 1.3 torr. For a complete experiment run time, the temperature of the cooling bath varied by $233 \pm 2 \mathrm{~K}$. The experimental details have been provided in Table 4.

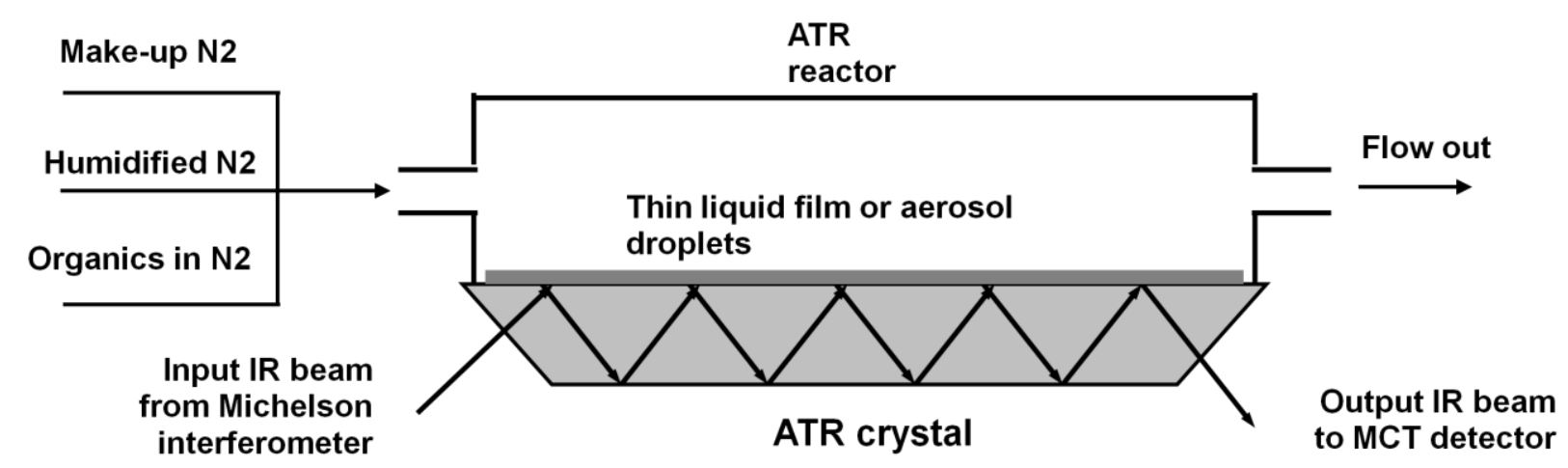

Figure 3: Schematic representation of ATR-FTIR set-up. 
Table 4: Experimental details for the ATR-FTIR experiments

\begin{tabular}{|c|c|c|c|}
\hline Epoxides & $\begin{array}{c}\text { Particle } \\
\text { composition }\end{array}$ & \multicolumn{2}{|l|}{ Experimental details } \\
\hline \multirow{14}{*}{ a-Pinene oxide } & \multirow{12}{*}{$\mathrm{H}_{2} \mathrm{SO}_{4}$} & \multirow{3}{*}{ RH (\%) (total flow rate 0.6 LPM) } & 9 \\
\hline & & & 42 \\
\hline & & & 84 \\
\hline & & \multirow{6}{*}{ Flow rates (LPM) (at RH 9\%) } & 0.3 \\
\hline & & & 0.6 \\
\hline & & & 1 \\
\hline & & & 2 \\
\hline & & & 2.5 \\
\hline & & & 3 \\
\hline & & \multirow{2}{*}{ Epoxide concentrations (at RH 9\%) } & $5.7 \times 10^{13}$ \\
\hline & & & $2.1 \times 10^{14}$ \\
\hline & & $\left(\right.$ molecules $\mathrm{cm}^{-3}$ ) & $4.5 \times 10^{14}$ \\
\hline & $\left(\mathrm{NH}_{4}\right)_{2} \mathrm{SO}_{4}$ & \multicolumn{2}{|c|}{$\begin{array}{c}\text { Dry (RH 60\%); total flow rate } 1 \text { LPM } \\
\text { Deliquesced (RH 80\%); total flow rate } 1 \text { LPM }\end{array}$} \\
\hline & $\mathrm{NH}_{4} \mathrm{HSO}_{4}$ & \multicolumn{2}{|c|}{ RH $60 \%$; total flow rate 1 LPM } \\
\hline \multirow{8}{*}{ Isoprene oxide } & \multirow{6}{*}{$\mathrm{H}_{2} \mathrm{SO}_{4}$} & \multirow{3}{*}{ RH (\%) (total flow rate 1 LPM) } & 13 \\
\hline & & & 41 \\
\hline & & & 80 \\
\hline & & & $1.25 \times 10^{14}$ \\
\hline & & Epoxide concentrations (at RH 13\%) & $4.2 \times 10^{14}$ \\
\hline & & & $8.4 \times 10^{14}$ \\
\hline & $\left(\mathrm{NH}_{4}\right)_{2} \mathrm{SO}_{4}$ & $\begin{array}{l}\text { Dry (RH 60\%); total flow rate } \\
\text { Deliquesced (RH 80\%); total flow }\end{array}$ & e $1 \mathrm{LPM}$ \\
\hline & $\mathrm{NH}_{4} \mathrm{HSO}_{4}$ & RH $60 \%$; total flow rate $1 \mathrm{~L}$ & \\
\hline
\end{tabular}




\subsection{NMR measurements}

${ }^{1} \mathrm{H}$ NMR hydrolysis kinetics measurements were performed using Inova-300 NMR spectrometer operating at $300 \mathrm{MHz}$. Three sets of experiments were carried out for each epoxide. For the first set, $25 \mu \mathrm{l}$ of epoxide was added to $5 \mathrm{ml}$ of $\mathrm{D}_{2} \mathrm{SO}_{4}$ solution of different concentrations, similar to other studies [Minerath and Elrod, 2009; Minerath et al., 2008; Minerath et al., 2009]. For the second set, $32 \mu \mathrm{l}$ of epoxide was added to $2 \mathrm{ml}$ of $\mathrm{D}_{2} \mathrm{SO}_{4}$ solution of different concentrations. For the third set, $100 \mu \mathrm{l}$ of epoxide was added to $1 \mathrm{ml}$ of $\mathrm{D}_{2} \mathrm{SO}_{4}$ solution of 5\% wt concentration. Another spectrum acquisition was run overnight, with $1 \mathrm{mg}$ of product in the solution, to keep it close to the product yield obtained in the ATR-FTIR experiments. All $\mathrm{D}_{2} \mathrm{SO}_{4}$ solutions were prepared using commercially available $\mathrm{D}_{2} \mathrm{SO}_{4}\left(96-98 \%\right.$, Sigma-Aldrich) diluted with $\mathrm{D}_{2} \mathrm{O}$. Since the solvent used was $\mathrm{D}_{2} \mathrm{O}, \mathrm{D}_{2} \mathrm{SO}_{4}$ was used instead of $\mathrm{H}_{2} \mathrm{SO}_{4}$ to avoid any ambiguity in peak identification in the acid catalyzed reaction. Acid solution was mixed with epoxide and allowed to stand for $5 \mathrm{~min}$. The solution was then transferred to a 5mm NMR tube and inserted into the spinner. All reactions were monitored at $296 \pm 2 \mathrm{~K}$. The experimental conditions for all sets (excluding overnight experiment) are shown in Table 5. 
Table 5: Experimental details for the NMR experiments

\begin{tabular}{|c|c|c|c|}
\hline \multirow{4}{*}{ Epoxide } & $\begin{array}{c}\text { Acid concentration } \\
(\text { wt } \%)\end{array}$ & Acid volume $(\mathrm{ml})$ & Epoxide volume $(\mu \mathrm{l})$ \\
\hline \multirow{5}{*}{$\alpha$-Pinene oxide } & $5 \%$ & 5 & 25 \\
\cline { 2 - 4 } & $40 \%$ & 5 & 25 \\
\cline { 2 - 4 } & $80 \%$ & 2 & 25 \\
\cline { 2 - 4 } & $0.02 \%$ & 2 & 32 \\
\cline { 2 - 4 } & $0.3 \%$ & 2 & 32 \\
\cline { 2 - 4 } & $0.7 \%$ & 5 & 32 \\
\cline { 2 - 4 } & $\mathrm{Na}_{2} \mathrm{SO}_{4} / 5 \%$ & 1 & 25 \\
\hline \multirow{5}{*}{ Isoprene oxide } & $5 \%$ & 5 & 25 \\
\cline { 2 - 4 } & $5 \%$ & 2 & 32 \\
\cline { 2 - 4 } & $0.7 \%$ & 1 & 100 \\
\hline
\end{tabular}




\subsection{Optical microscope measurements}

Several measurements were made employing the Raman optical microscope in order to support the results from the ATR-FTIR measurements. Round silica chips of diameter 0.9 $\mathrm{mm}$ were used for analyses. These silica chips were placed right over the ZnSe crystal and sulfuric acid aerosols were deposited on the crystal and silica chips as described before. The crystal along with the chips was placed inside the instrument and the same procedures were followed for taking the spectra as in the other FTIR measurements. After the experiment, silica chips were placed below the optical lens of the Raman optical microscope and analyzed. Images and spectra for all the samples were observed and recorded. All observations were made using the $10 \mathrm{X}$ and $50 \mathrm{X}$ objectives of the Thermo-Scientific DXR Raman Microscope. 


\section{RESULTS AND DISCUSSION}

\subsection{Uptake measurements using ID-CIMS}

All the uptake measurements performed using the low pressure laminar flow ID-CIMS showed that the reaction between the epoxide and sulfuric acid was irreversible. In each experiment, epoxide was passed through the movable injector till a steady-state flow was established downstream of the trough containing acid. The signal of epoxide was continuously monitored. Then the injector was pulled upstream to expose a certain length of the acid to epoxide. The length of exposure varied from $2 \mathrm{~cm}$ to $12 \mathrm{~cm}$. On exposure, the epoxide was taken up by the acid heterogeneously leading to product formation. At this stage, depending on temperature and acid concentration, either a drop in the epoxide signal can be observed indicating uptake and formation of low volatile products that stay in the solution, or an increase in signal can be observed due to one or more fragment ions coming from a more volatile product of the heterogeneous reaction. A decrease in the signal was observed only for experiments carried out at room temperature using $96 \% \mathrm{wt}$ acid. There was no recovery in the signal over the time scale of exposure. When the injector was pushed back downstream the acid surface, the epoxide signal recovered to its original level. Other acid concentrations were not tried at room temperatures due to difficulties in maintaining water vapor pressure of acid at high temperatures. At lower temperatures, there was reduced loss of water from acid solution and it was easier to maintain uniform acid concentration by addition of water vapor [R. Zhang et al., 1993]. The lower temperatures tried were 283K, $263 \mathrm{~K}$ and $243 \mathrm{~K}$. For all uptake experiments at lower temperatures an increase in signal was observed on exposing acid to epoxide. This observation was made for all reagent ions that were successful in detection of epoxide. 


\subsubsection{Isoprene oxide}

In case of isoprene oxide, using $\mathrm{H}_{3} \mathrm{O}^{+}$as reagent ion, different drift tube voltages were tried to obtain a representative fragment of the epoxide molecule. Since fragmentation increased due to increasing drift tube voltage, a suitable voltage was required to monitor the protonated epoxide molecule and avoid formation of several fragments. At a selected voltage, the ions with $\mathrm{m} / \mathrm{z} 43$ and 85 were monitored. Both these ions decreased by $\sim 2 \%$ in intensity on exposure to acid. At lower temperatures, $\mathrm{m} / \mathrm{z} 43$ showed a decrease in signal by $<1 \%$, but $\mathrm{m} / \mathrm{z} 85$ increased on exposure to acid. Since the signal for $\mathrm{m} / \mathrm{z} 85$ increased, and the decrease in $\mathrm{m} / \mathrm{z} 43$ was not significant, the reagent ion was considered unsuitable for differentiating between the epoxide and the volatile product with similar $\mathrm{m} / \mathrm{z}$ values. Hence, a different reagent ion was tried. With $\mathrm{O}_{2}^{-}, \mathrm{m} / \mathrm{z} 116$ was monitored. This signal also decreased at room temperature by $\sim 50 \%$ but increased at lower temperatures for all acid concentrations. This increase at $283 \mathrm{~K}$ using $75 \%$ wt acid was $\sim 67 \%$, as shown in Figure 4 . With $\mathrm{O}_{2}{ }^{+}, \mathrm{m} / \mathrm{z} 84$ and 69 were monitored. These ions showed a decrease at lower temperature, but the decrease was $\sim 10 \%$ at exposure distance $2 \mathrm{~cm}$ and did not change on further exposure. All the ions detected using different reagent ions had same $\mathrm{m} / \mathrm{z}$ as the protonated epoxide or a fragment from epoxide. Most likely, there was interference from some product ion that had same $\mathrm{m} / \mathrm{z}$ as epoxide and all the reagent ions tried were not able to differentiate between the epoxide and the product ion. From the measurements made at room temperature using $96 \%$ wt acid, the uptake coefficient $\gamma$ of isoprene oxide (considering $\mathrm{m} / \mathrm{z} 116$ ) was calculated to be $4 \times 10^{-2}$. 


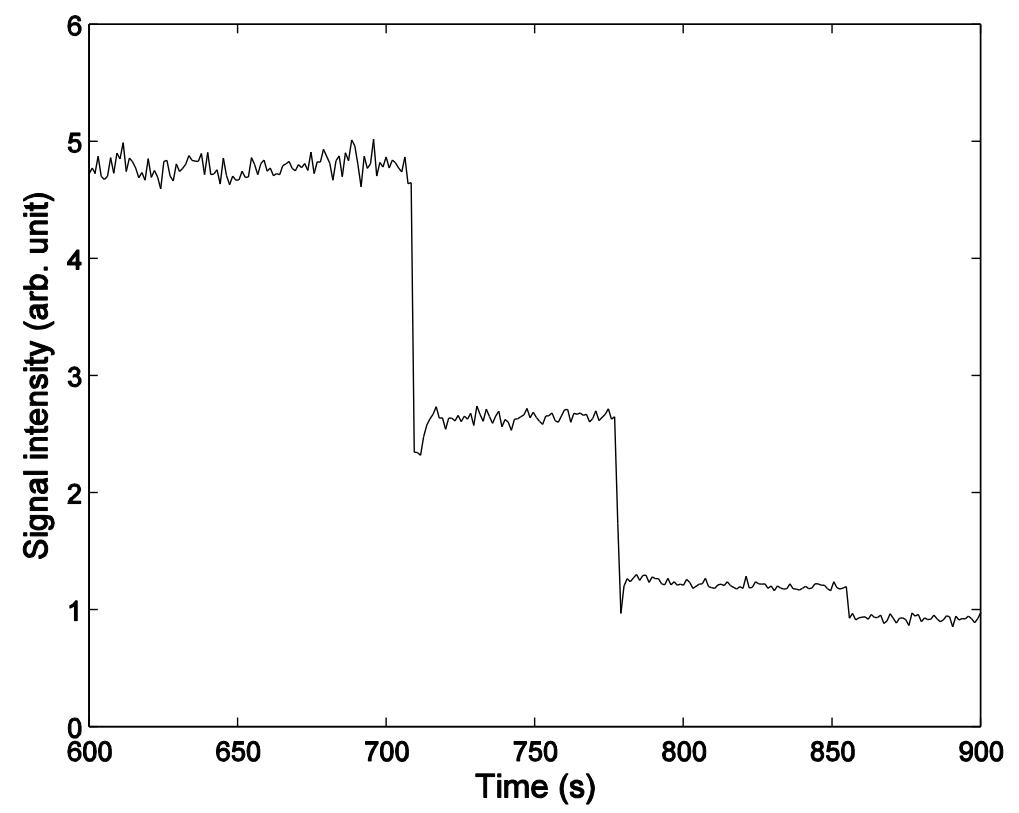

(a)

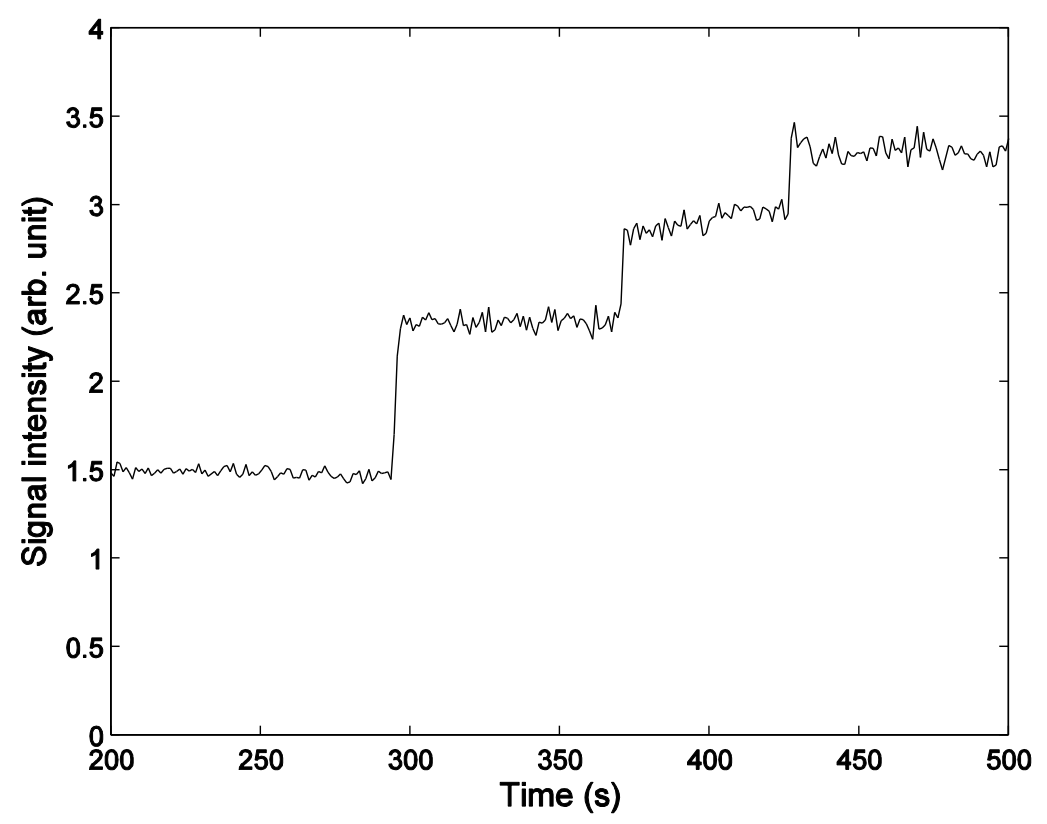

(b)

Figure 4: Temporal profiles of $\mathrm{m} / \mathrm{z} 116$ under different conditions using $\mathrm{O}_{2}{ }^{-}$as reagent ion (a) isoprene oxide cluster with $\mathrm{O}_{2}^{-} \mathrm{m} / \mathrm{z} 116$ decreases on exposure to $96 \%$ wt acid with $\mathrm{T}=298 \mathrm{~K}$, $\mathrm{P}=1.68$ torr, $\mathrm{u}=664 \mathrm{~cm} \mathrm{~s}^{-1}$. (b) Hydroxy alkene/aldehyde cluster with $\mathrm{O}_{2}^{-} \mathrm{m} / \mathrm{z}$ at $283 \mathrm{~K}$. 
In order to understand the interference by other ions with same $\mathrm{m} / \mathrm{z}$ as epoxide or a fragment of epoxide, some bulk measurements were carried out. Isoprene oxide was mixed with $75 \%$ wt acid in 1:100 ratio at room temperature. An exothermic reaction led to the formation of a deep yellow-brown solution almost instantly. It was assumed that the epoxide had completely reacted with the acid, due to its high reactivity and hydrolysis rate constant [Minerath et al., 2009]. This solution was allowed to stand for an hour. It was then placed in the trough and monitored using $\mathrm{H}_{3} \mathrm{O}^{+}, \mathrm{O}_{2}{ }^{-}$and $\mathrm{O}_{2}{ }^{+}$at $243 \mathrm{~K}$. Using $\mathrm{H}_{3} \mathrm{O}^{+}$and $\mathrm{O}_{2}{ }^{+}, \mathrm{m} / \mathrm{z} 85$ was again observed, proving that one of the volatile product ions had the same $\mathrm{m} / \mathrm{z}$ as epoxide. Using $\mathrm{O}_{2}{ }^{-}$, the cluster ion with $\mathrm{m} / \mathrm{z} 116$ was observed along with several other ions, thus supporting the results from other reagent ions. The only major difference from uptake experiments was absence of $\mathrm{m} / \mathrm{z} 43$ in bulk experiments using $\mathrm{H}_{3} \mathrm{O}^{+}$as reagent ion. To investigate the role of sulfuric acid concentration on the ratio of $\mathrm{m} / \mathrm{z} 43$ and 85 , a small volume of epoxide was mixed with deionized water in 1:200 ratio at room temperature. A colorless solution was formed. The solution was allowed to stand overnight to ensure complete reaction. An ATRFTIR spectrum of this solution was taken to confirm completion of the reaction. The solution was then placed in the trough and monitored using $\mathrm{H}_{3} \mathrm{O}^{+}$at $243 \mathrm{~K}$. Strong signals were obtained for $\mathrm{m} / \mathrm{z} 43$ and 85 . Stepwise addition of $75 \%$ wt acid was made to the solution and for every addition, both ions 43 and 85 were monitored. Figure 5 shows the change in these two ions with increasing acidity. 


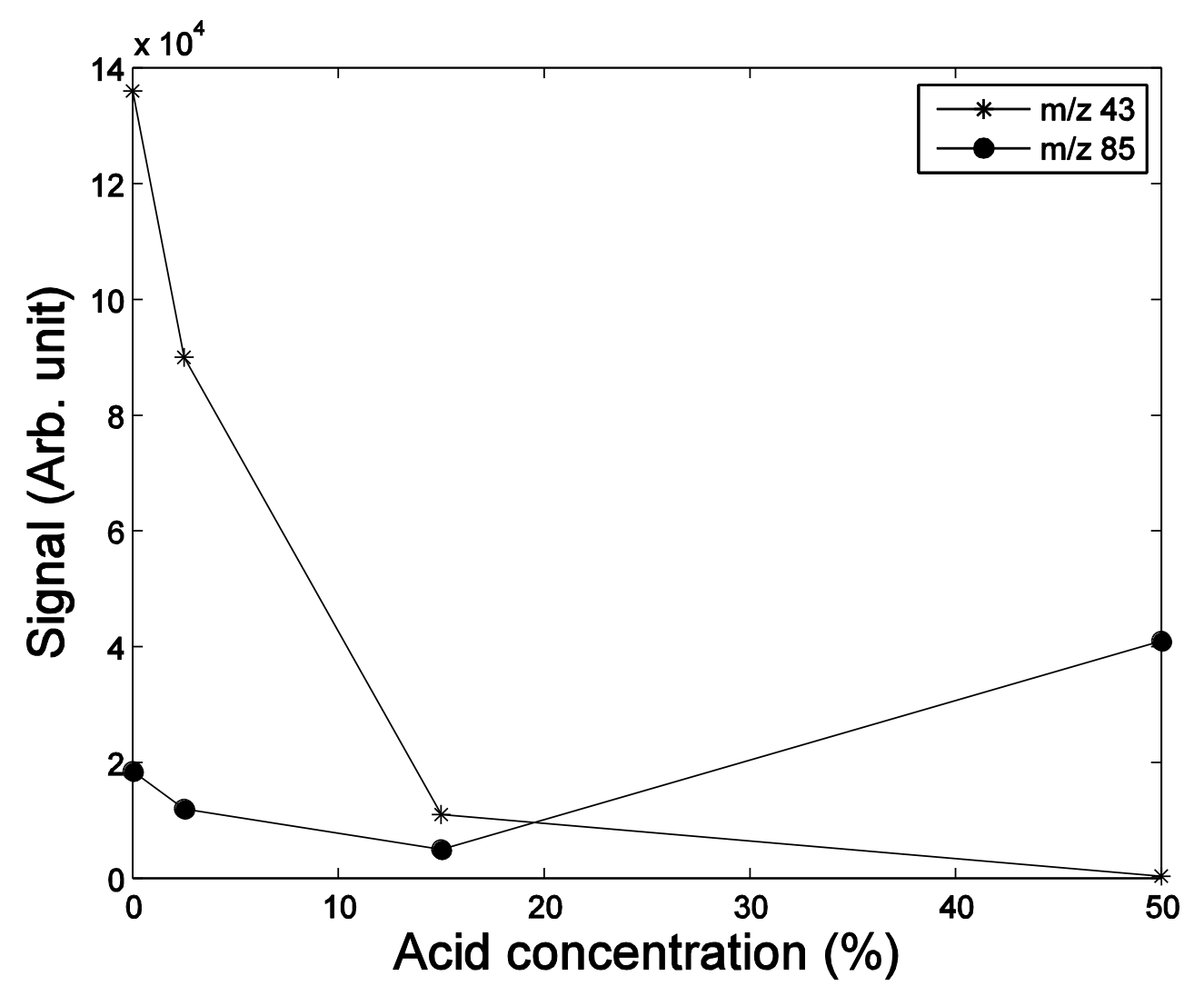

Figure 5: Response of ions $\mathrm{m} / \mathrm{z} 43$ and 85 on successive addition of acid to epoxide-water mixture.

Ion with $\mathrm{m} / \mathrm{z} 43$ was seen to decrease with increasing acidity, while $\mathrm{m} / \mathrm{z} 85$ decreased initially but later increased with increasing acidity. Since epoxide reacts with water to form diol, and with a high hydrolysis reaction rate constant of isoprene oxide with water ( $\mathrm{k}=56000$ $\mathrm{M}^{-1} \mathrm{~s}^{-1}$ ) [Minerath et al., 2009], it was evident that both $\mathrm{m} / \mathrm{z} 43$ and 85 represented diol which was volatile enough to slowly escape the solution and produce a signal. On addition of acid, diol can react to form sulfate ester [Minerath et al., 2008], or can form other condensation products. This resulted in decreased signals of $\mathrm{m} / \mathrm{z} 43$ and $\mathrm{m} / \mathrm{z} 85$. Addition of more acid eventually led to an increased $\mathrm{m} / \mathrm{z} 85$ signal which came from a compound that was again volatile to leave the solution. There could be two possibilities for the formation of this 
volatile product. As the acidity of the solution increases, the diol (with a tertiary alcohol group) can dehydrate and form hydroxy-alkene which is volatile enough to escape the condensed phase. Previous studies on reaction of alcohols with sulfuric acid [Clark and Williams, 1957; Minerath et al., 2008] show that alcohols react with sulfuric acid to form ester and the reaction is exothermic. Also, secondary and tertiary alcohols dehydrate in the presence of acid to form alkenes which are too volatile to stay in solution. The ester forming reaction has large activation energy and hence leads to reduced sulfate ester formation at lower temperatures. The vapor pressure of the sulfate ester being low, it always stays in solution. So at room temperature, sulfate ester is formed by the uptake of epoxide and hence a decrease in signal is observed. At lower temperatures, the sulfate ester forming tendency decreases and instead the diol formed dehydrates to give hydroxy-alkene. An increase in the $\mathrm{m} / \mathrm{z} 85$ (or 116) is because of the release of this alkene which has the same $\mathrm{m} / \mathrm{z}$ as epoxide.

Another possibility can be dehydration of the diol and rearrangement to form an aldehyde. Epoxides and alcohols in presence of acid protonate and form a carbocation (mostly secondary or tertiary) which rearranges into an aldehyde [Ley and Vernon, 1957; Parker and Isaacs, 1959]. At room temperature, the aldehydes can react with diols to form acetals, or undergo aldol condensation. Aldol condensation reaction possibility is positively correlated with temperature, and hence at lower temperatures, the reaction is not favorable [Casale et al., 2007]. The volatile aldehydes instead of participating in other reactions therefore move into the gas phase and contribute to the increased signal of $\mathrm{m} / \mathrm{z} 85$ (or 116). The formation mechanisms of these volatile products are explained in Figure 6. 


\subsection{2 $\alpha$-Pinene oxide}

Uptake measurements for $\alpha$-pinene oxide were also carried out using similar procedure, with $\mathrm{H}_{3} \mathrm{O}^{+}$and $\mathrm{O}_{2}^{-}$as reagent ions. Using $\mathrm{H}_{3} \mathrm{O}^{+}$, ions with $\mathrm{m} / \mathrm{z} 43$ and 93 were monitored. $\mathrm{m} / \mathrm{z} 93$ fragment ion (most likely $\mathrm{C}_{3} \mathrm{H}_{7}{ }^{+}$) has been observed in several PTR-MS studies of monterpenes, sesquiterpenes and monoterpene oxides [Ambrose et al., 2010; Dhooghe et al., 2008; Maleknia et al., 2007; Schoon et al., 2003; Schoon et al., 2004; A. Tani et al., 2003; Akira Tani et al., 2004; Warneke et al., 2003]. At $283 \mathrm{~K}$ using 75\% wt acid, the signal strength for these ions was extremely low and an increase or decrease on exposure to acid was not identifiable. Using $\mathrm{O}_{2}{ }^{-}, \mathrm{m} / \mathrm{z} 184$ was monitored. At $283 \mathrm{~K}$ using $75 \%$ wt acid, the signal for $\mathrm{m} / \mathrm{z} 184$ increased on exposure to acid. Thus, there was interference again from a product ion which could not be differentially detected by any of the reagent ions used. From the preliminary measurements made for $\alpha$-pinene oxide at $298 \mathrm{~K}$ and using $96 \% \mathrm{wt}$ acid, the $\gamma$ obtained was $0.8 \times 10^{-2}$.

Bulk experiments for $\alpha$-pinene oxide were carried out and the reaction mixture containing epoxide and acid was tested using $\mathrm{H}_{3} \mathrm{O}^{+}$and $\mathrm{O}_{2}^{-}$. With $\mathrm{H}_{3} \mathrm{O}^{+}, \mathrm{m} / \mathrm{z} 93$ was observed. With $\mathrm{O}_{2}^{-}$, small amount of the cluster ion $\mathrm{m} / \mathrm{z} 184$ was observed, but several other ions are formed which could result from fragmentation. This supports our observation for isoprene epoxide and confirms the formation of a volatile compound (alkene or aldehyde) that interferes with the epoxide signal and increases at lower temperatures. The isomerization of $\alpha$-pinene oxide under acidic conditions to form alkenes and aldehydes has been described in previous studies [Carr et al., 1994; Iinuma et al., 2009]. 
From the uptake and bulk experiments carried out using ID-CIMS, it was concluded that the reaction of epoxides with acid is very rapid and irreversible. At room temperature and high acidic conditions, uptake of epoxide was observed showing a decrease in signal. This uptake could result in formation of diols, sulfate esters and condensation products like aldol or acetal. These are all low volatility products which stay in solution. But at lower temperatures even with high acidic conditions, formation of esters, aldol and acetal, though thermodynamically favorable, are kinetically not very feasible. Instead dehydration of diol is preferred which leads to formation of hydroxy-alkene or rearranges to form aldehydes. These high volatile compounds do not stay in the solution and evaporate into gas phase. Both these classes of compounds can be detected by the reagent ions employed in our experiments [McDonald and Chowdhury, 1985; Zhao et al., 2006], and hence the interference was observed. 


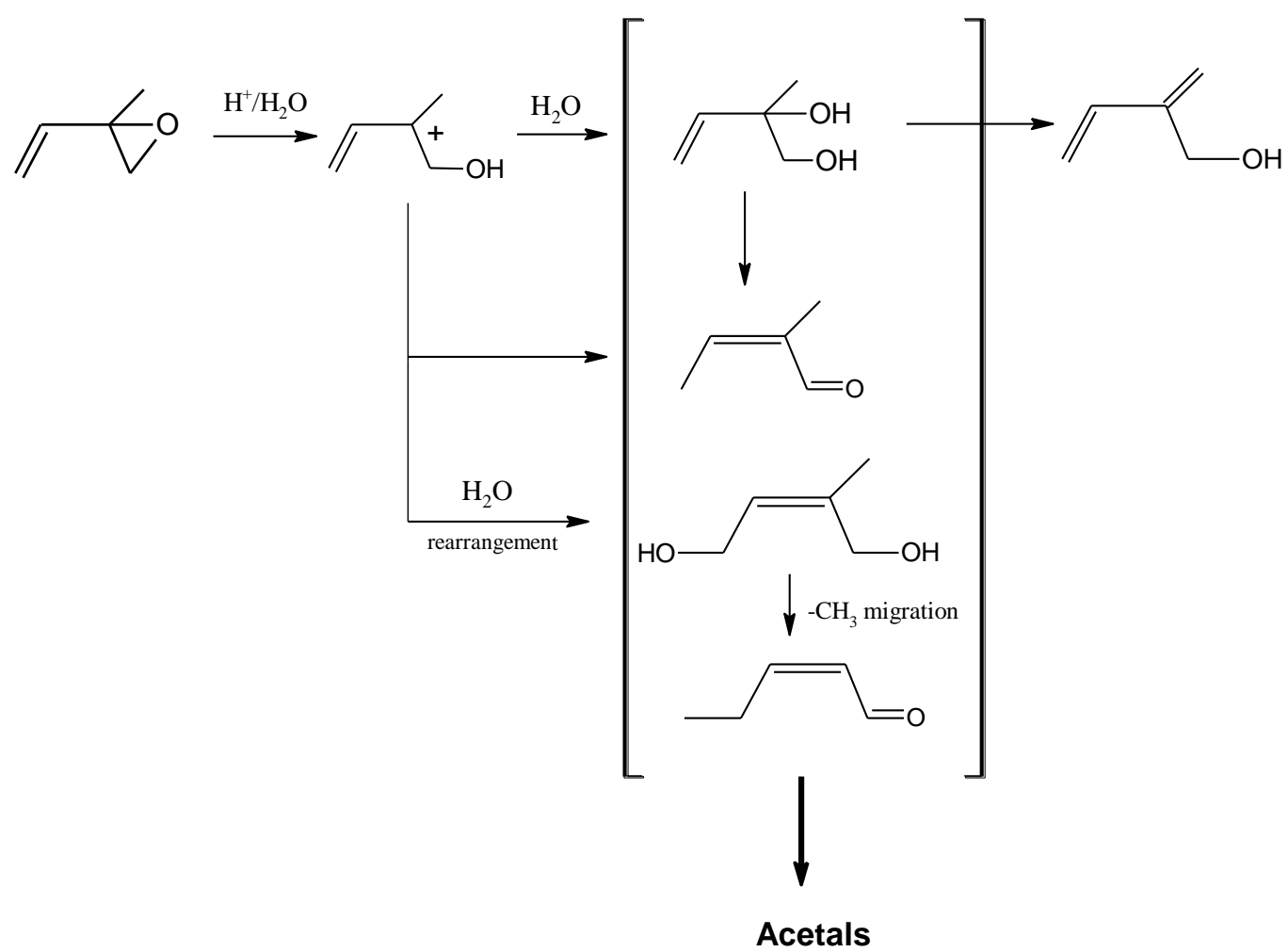

(a)

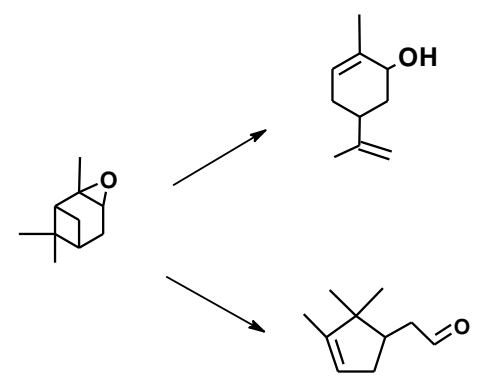

(b)

Figure 6: (a) Acid catalyzed products for isoprene oxide. (b) Acid-catalyzed isomerization products for $\alpha$-pinene oxide with same $\mathrm{m} / \mathrm{z}$ as the epoxide. 


\subsection{ATR-FTIR measurements}

Attenuated total reflectance Fourier transform infrared spectroscopy (ATR-FTIR) was used to investigate the acid catalyzed heterogeneous reactions of epoxides. This versatile technique is useful for detection of highly absorbing solids and liquids mainly due to easier sample preparation and small penetration depth of the evanescent wave generated by internal reflection of IR beam [Schuttlefield and Grassian, 2008]. It works on the principle of total internal reflection that takes place when a light beam from the spectrometer moves from a medium with higher refractive index to a medium with lower refractive index at an angle of incidence greater than the critical angle. An evanescent wave is produced at every reflection which has a short penetration depth to the order of a few micrometers. The penetration depth can be calculated using the following equation:

$$
d_{p}=\frac{\lambda}{2 \pi\left(n_{1}^{2} \sin ^{2} \theta-n_{2}^{2}\right)^{1 / 2}}
$$

where, $\lambda=$ wavelength of light, $\theta=$ angle of incidence of the IR beam, $\mathrm{n}_{1}=$ refractive index of the crystal and $\mathrm{n}_{2}=$ refractive index of the sample. The intensity of this evanescent wave is attenuated where the radiation is absorbed by the sample. The absorption of IR radiation by the sample is measured in terms of absorbance. This absorbance is directly proportional to the path length, more specifically called the effective path length in ATR-FTIR spectroscopy. The Beer Lambert's Law relates the absorbance to concentration of the sample and the effective path length by the following relation:

$$
A=\varepsilon c b^{\prime}
$$


where $\mathrm{A}$ is the absorbance, $\mathrm{b}$ is the effective path length, $\mathrm{c}$ is the concentration and $\varepsilon$ is the molar absorptivity. The effective path length is equal to the number of reflections times the penetration depth.

In our case the refractive index $\left(\mathrm{n}_{1}\right)$ of the $\mathrm{ZnSe}$ crystal was 2.4 with angle of incidence equal to $45^{\circ}$. Most organics have been found to have a refractive index between 1.4-1.8 [Baumgardner et al., 2007; Katritzky et al., 1998]. Hence the penetration depth was assumed to be of the order of 2-3 $\mu \mathrm{m}$. The layer of sulfuric acid aerosols deposited on the ZnSe crystal was within this penetration depth limit, as revealed by the SEM and optical microscope images. Figure 22 in Appendix represents the sulfuric acid spectra at different RH.

\subsection{1 $\quad \alpha$-Pinene oxide}

Experiments with $\alpha$-pinene oxide were carried out under conditions of different RH, epoxide concentrations and flow rates. Figure 7 shows the ATR-FTIR absorbances for $\alpha$-pinene oxide reaction with sulfuric acid at different $\mathrm{RH}$. Detailed peak identification for the fingerprint region is provided in Table 6 . The strong broad peak at $3350 \mathrm{~cm}^{-1}$ was assigned to the $\mathrm{O}-\mathrm{H}$ stretch. The intensity of this peak was seen to reduce with decreasing RH. The peaks in the region $3000-2800 \mathrm{~cm}^{-1}$ were due to $\mathrm{C}-\mathrm{H}$ stretch vibrations. The $\mathrm{C}-\mathrm{H}$ peaks observed in all the 3 cases were at similar frequencies and hence showed the ring structure to be preserved. The fingerprint region was complicated due to the presence of several overlapping peaks from different functional groups. 


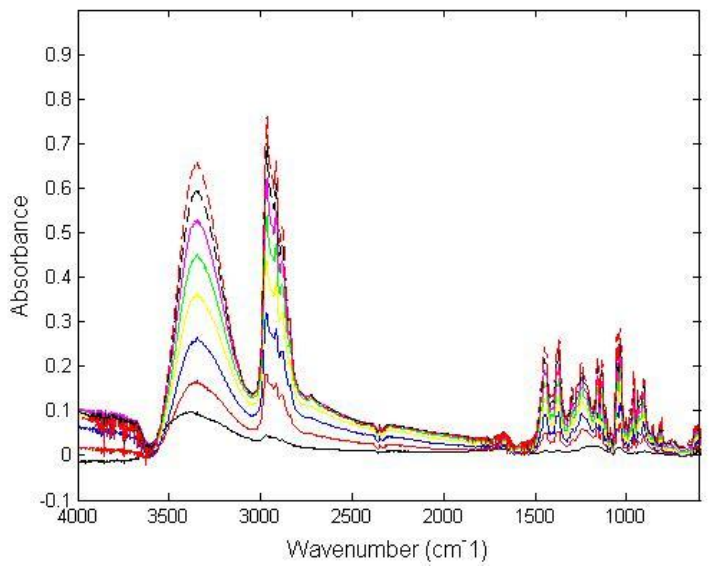

(a)

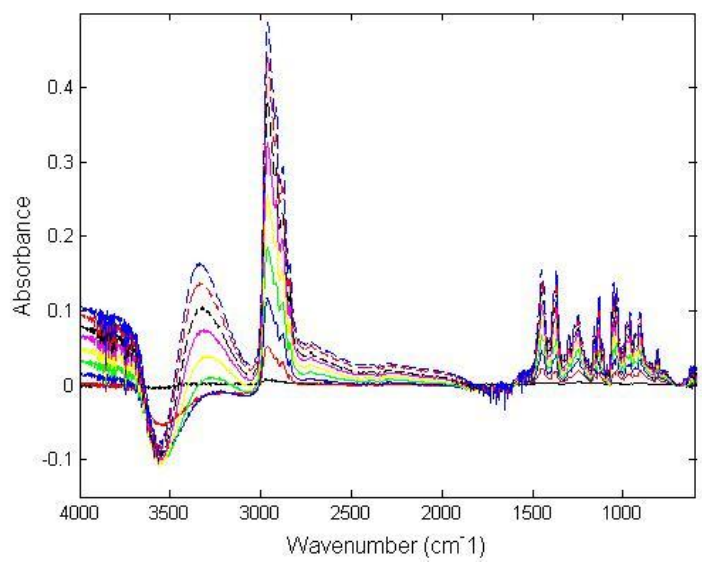

(b)

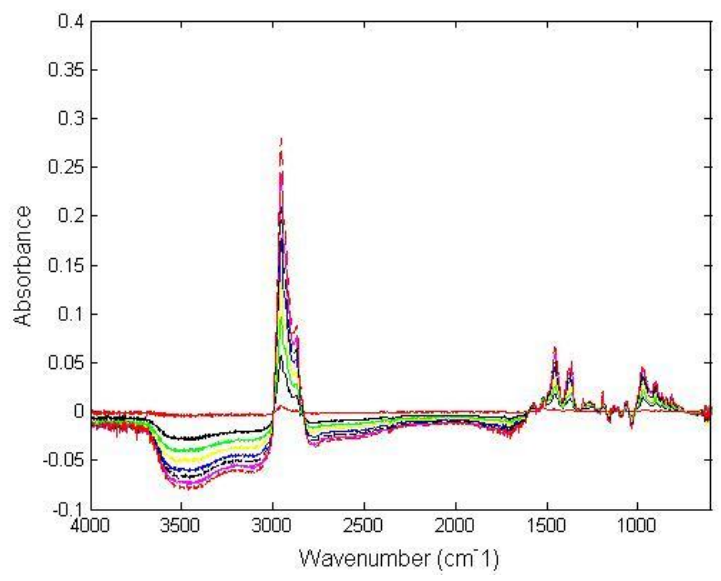

(c)

Figure 7: ATR-FTIR spectra of $\alpha$-pinene oxide reaction with sulfuric acid at (a) $84 \%$ RH (b) $42 \% \mathrm{RH}$ (c) $9 \% \mathrm{RH}$. Total time for the reactions was $70 \mathrm{~min}$ in each case. 
Table 6: ATR-FTIR absorption frequencies assigned to the functional groups formed by acid hydrolysis of $\alpha$-pinene oxide

\begin{tabular}{|c|c|c|c|}
\hline \multirow{2}{*}{$\begin{array}{l}\text { Assignment } \\
\text { of frequency }\end{array}$} & \multicolumn{3}{|c|}{ Frequencies $\left(\mathrm{cm}^{-1}\right)$} \\
\hline & RH $84 \%$ & RH $42 \%$ & RH 9\% \\
\hline O-H stretch & 3349 & 3372 & Not significant \\
\hline C-O stretch (secondary alcohol $/{ }^{*}$ sulfate) & 1037 & 1058 & $1070^{*}$ \\
\hline $\mathrm{O}-\mathrm{H}$ in-plane deformation $/^{*}$ sulfate & 1247 & 1262 & $1267^{*}, 1194^{*}$ \\
\hline $\mathrm{C}-\mathrm{O}$ stretch (tertiary alcohols) & 1135,1159 & 1202 & 1148 \\
\hline S-O stretch & - & - & 872 \\
\hline
\end{tabular}




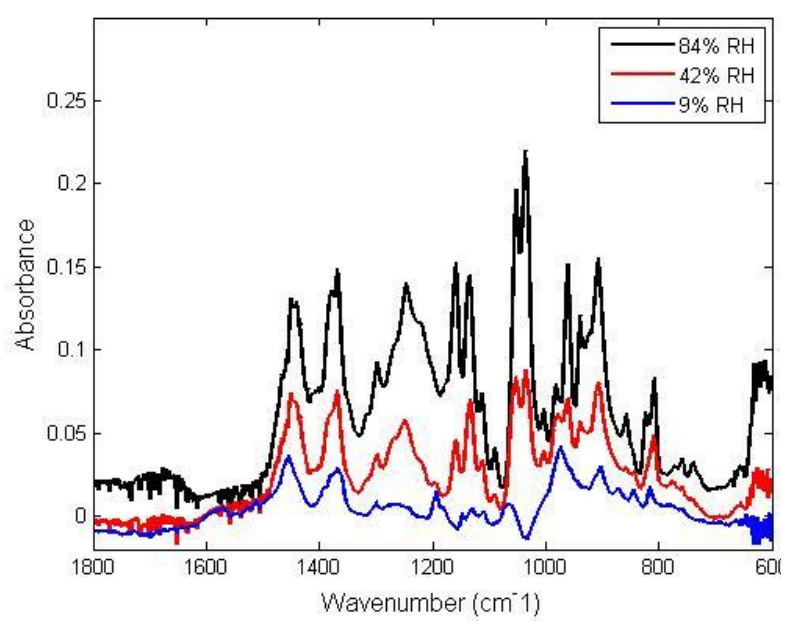

(a)

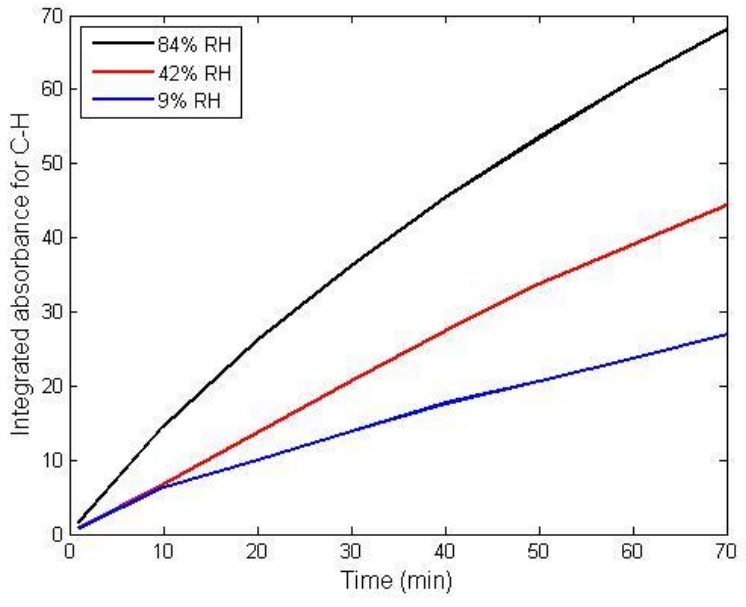

(b)

Figure 8: (a) ATR-FTIR spectra in the fingerprint region for the product of acid-epoxide reaction (for $\alpha$-pinene oxide) under different humidity conditions. (b) Integrated C-H absorbance (3000-2850 $\mathrm{cm}^{-1}$ ) over time for $\alpha$-pinene oxide at different $\mathrm{RH}$.

The region from $1400-1300 \mathrm{~cm}^{-1}$ includes contributions both from the ring as well as alcohols. At $84 \% \mathrm{RH}$, the strong peaks at 1136 and $1037 \mathrm{~cm}^{-1}$ represent the $\mathrm{C}-\mathrm{O}$ stretch for tertiary and secondary alcohols, respectively. Peaks in the region $900-800 \mathrm{~cm}^{-1}$ represent CC-O stretch for secondary alcohol group and in the region $800-750 \mathrm{~cm}^{-1}$ for tertiary alcohol group. However, at $9 \% \mathrm{RH}$, the peak at $1037 \mathrm{~cm}^{-1}$ is replaced by a short broad peak at 1066 $\mathrm{cm}^{-1}$ which is assigned to the $\mathrm{SO}_{2}$ symmetric stretch, and a moderately strong peak at 1194 $\mathrm{cm}^{-1}$ to the $\mathrm{SO}_{2}$ asymmetric stretch. The asymmetric S-O-C stretching vibration occurs at 872 $\mathrm{cm}^{-1}$ represented by a peak of medium intensity [Gilardoni et al., 2007; Socrates, 2004]. The IR spectrum at $42 \%$ RH shows the presence of diol and a small amount of sulfate. The sulfate peak intensity is too low for detection. With decreasing RH, and hence increasing acidity, the concentration of sulfate in the solution increases and sulfate esters are produced. The decrease in $\mathrm{O}-\mathrm{H}$ absorbance and hence loss of $\mathrm{O}-\mathrm{H}$ groups supports this observation. The 
overall absorbance at $9 \% \mathrm{RH}$ is very low and it increases with increasing RH. The temporal evolution of products as a function of RH is seen in Figure 8. This observation can be explained with the help of optical microscopic images. The images in Figure 9 show that at high RH, the sulfuric acid drops lose their identity and spread out on the surface like a layer, while they stay intact at low humidity conditions. The spreading is observed only when the acid is exposed to epoxide at high humidities. In absence of epoxide, the spreading is not observed at similarly high humidity. Also, the spreading features show patterns of light interference. These observations can be attributed to increased wettability due to the products formed at high RH. Studies show that the intensity of IR absorption increases when the contact of sample with the crystal increases [Gao and Chorover, 2010]. An increase in water activity at high RH leads to more product formation and hence more wettabililty with the ZnSe crystal surface. These organics formed act as surfactants and decrease the contact angle with the crystal, thus increasing absorbance. At low RH conditions, water activity is reduced. Further, organosulfate formation is preferred for $\alpha$-pinene oxide due to more acidic sulfate available. Therefore, loss of $\mathrm{O}-\mathrm{H}$ absorbance is seen and less products formed result in reduced intensity of peaks. Relative to the alkyl absorbance at high $\mathrm{RH}$ conditions, no increase in intensity is observed at low RH conditions which imply that there is no carbon chain increment and hence, condensation products like aldol and acetal are not formed. 


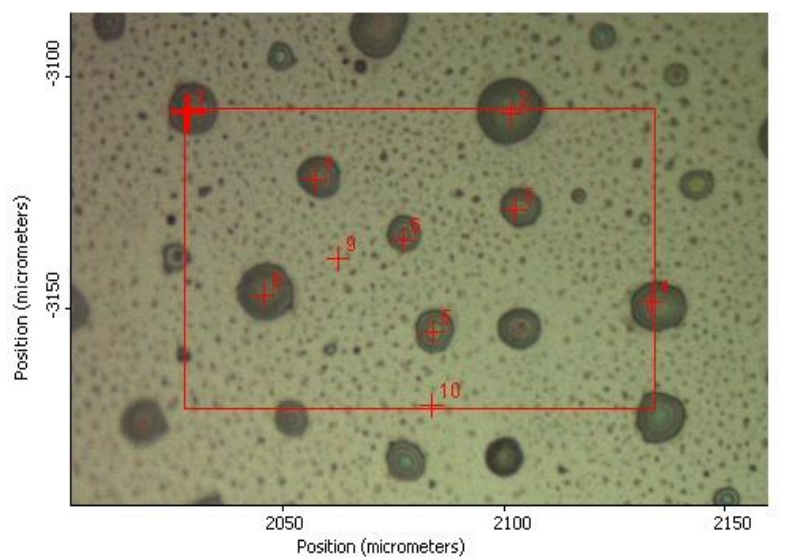

(a)

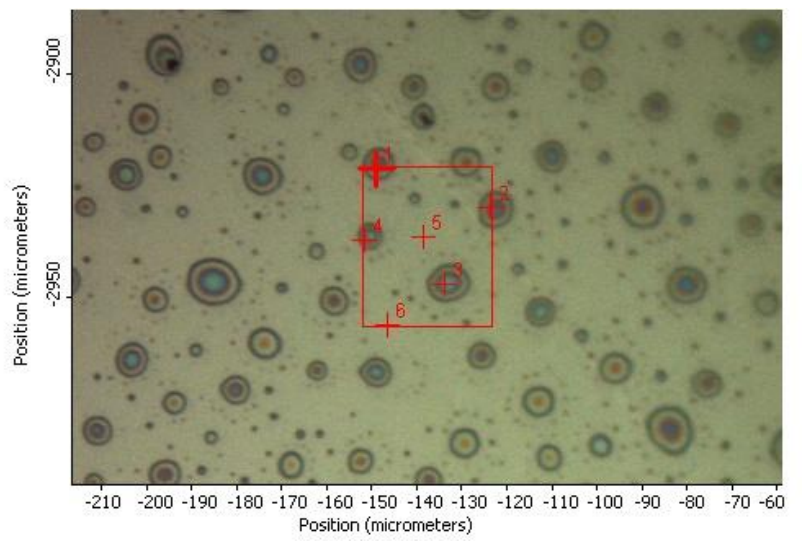

(c)

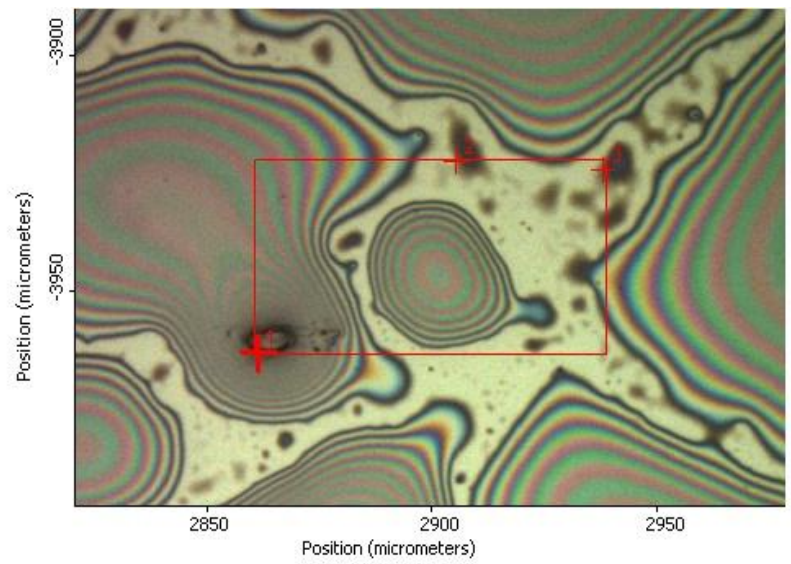

(b)

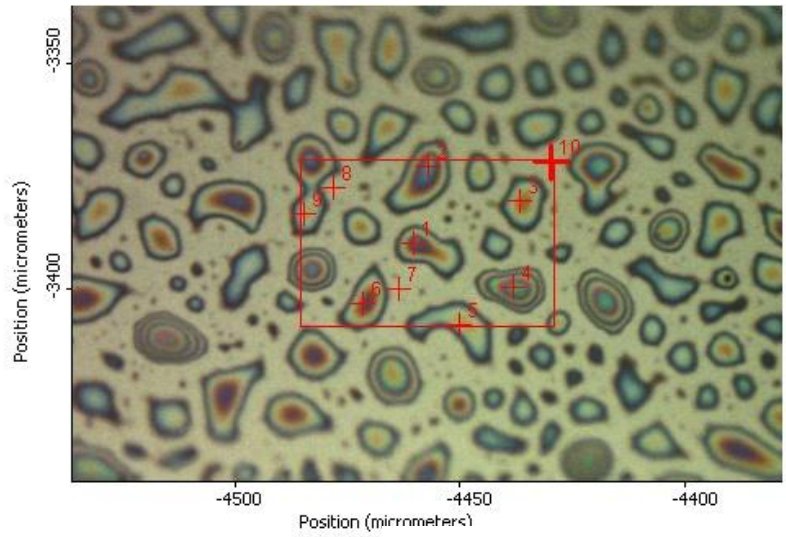

(d)

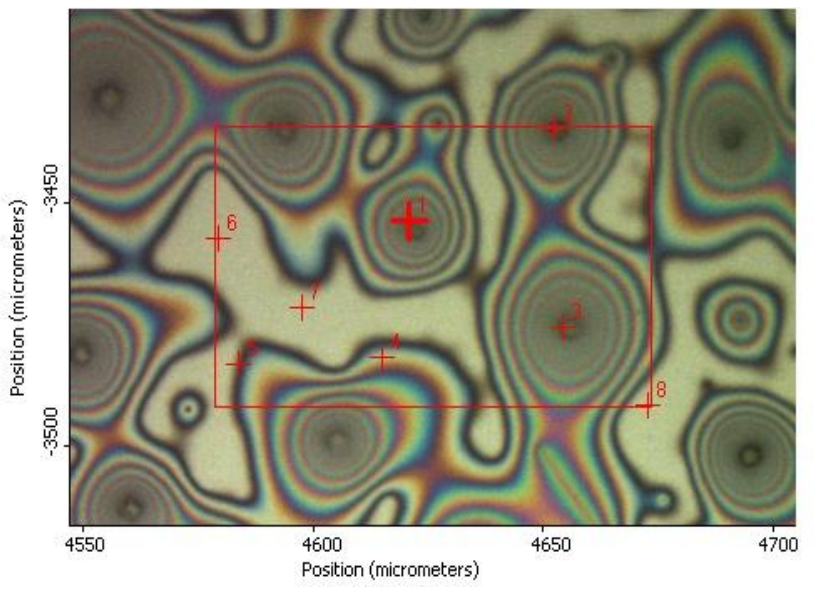

(e)

Figure 9: Optical microscopic images of sulfuric acid reaction with $\alpha$-pinene oxide (a) at 9\% RH after $30 \mathrm{~min}$ (b) at $84 \% \mathrm{RH}$ after $30 \mathrm{~min}$. Other images include (c) sulfuric acid exposed to water vapor only at $84 \% \mathrm{RH}$ after $30 \mathrm{~min}$ (d) sulfuric acid exposed to epoxide at $80 \% \mathrm{RH}$ after 5 min (e) ammonium bisulfate exposed to epoxide after $30 \mathrm{~min}$. 
Evidence for organosulfate production under acidic conditions for epoxides has been provided in previous studies [Minerath and Elrod, 2009; Minerath et al., 2009]. Organosulfate could possibly be produced by esterification of the alcohol group in the epoxide hydrolysis product [Minerath et al., 2008], or by direct attack of $\mathrm{HSO}_{4}{ }^{-}$nucleophile on the epoxide cation in competition with $\mathrm{OH}^{-}$(following the SN1 mechanism) [Iinuma et al., 2009; Iinuma et al., 2007; Minerath and Elrod, 2009]. The esterification reaction is fastest for the primary alcohol and slowest for tertiary alcohol [Minerath et al., 2008]. In case of $\alpha$-pinene oxide, the most probable products formed by the two mechanisms are shown in Figure 10.

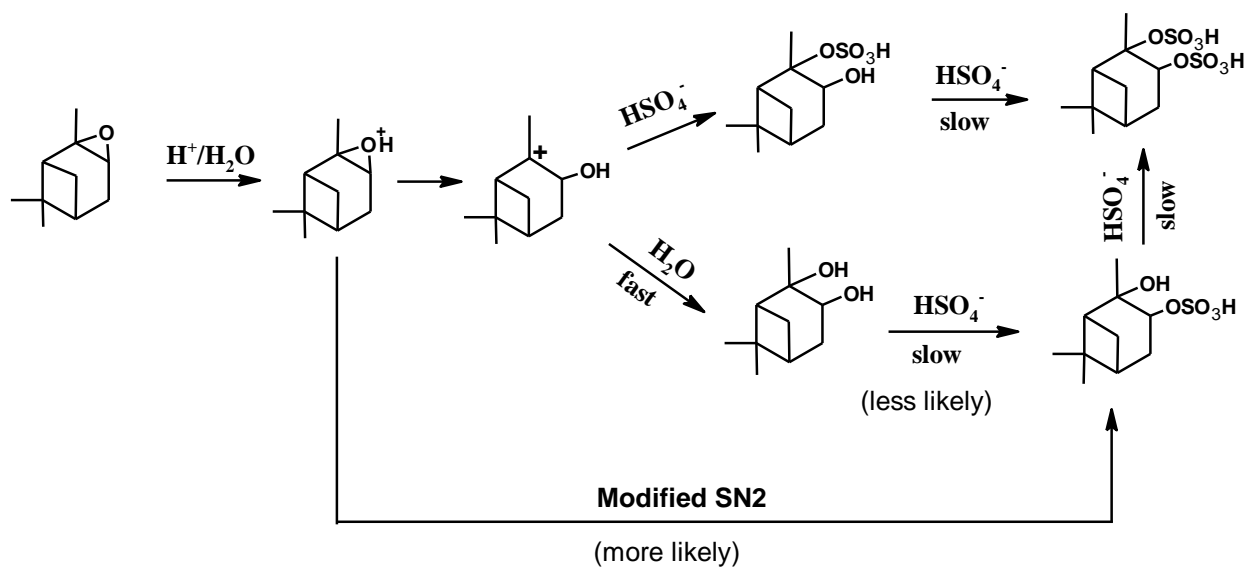

Figure 10: Formation of organosulfate by $\alpha$-pinene oxide under acidic conditions.

The structure of products have been inferred from the reaction mechanisms of epoxides and alcohols, and well supported by other studies [Iinuma et al., 2009]. Absence of other functional groups like $\mathrm{C}=\mathrm{O}$ and $\mathrm{C}=\mathrm{C}$ confirmed that there was no rearrangement of $\alpha$-pinene oxide under these experimental conditions. Yield of organosulfates was seen to increase with 
increasing acidic sulfate concentration in the aerosols. In our case at humidities greater than $40 \%$, the acid concentration was low for any organosulfate production. With increase in acidity at lower humidities, the $\left[\mathrm{HSO}_{4}{ }^{-}\right]$and $\left[\mathrm{SO}_{4}{ }^{2-}\right]$ concentrations also increased and hence contributed to formation of small amounts of organosulfate. Considering the above mechanisms, it can be concluded that the likely products formed would be diol at low acid concentrations and increasing sulfate ion concentration in the solution would eventually lead to formation of hydroxy-sulfate and disulfate ester products.

Figure 11 shows the results of experiments of $\alpha$-pinene oxide reaction with sulfuric acid carried out at different flow rates. In the uptake experiments using low pressure ID-CIMS, the reaction rate is limited by uptake of epoxide by sulfuric acid, since diffusion is faster than the chemical reaction. But in ATR-FTIR experiments, diffusion becomes the limiting factor since it is much slower at atmospheric pressure. For such cases, $\mathrm{k}>\mathrm{k}_{\mathrm{diff}}$ and hence the observed reaction rate constant is totally controlled by the diffusion rate constant. To determine the diffusion limitation and the flow rate at which it can be overcome, several experiments were carried out at different flow rates. All the flow variation experiments were conducted both at $42 \% \mathrm{RH}$ and $9 \% \mathrm{RH}$ conditions. The plots indicate that the reaction is indeed diffusion limited for flow rates below 2 LPM, irrespective of RH. At higher flow, saturation of epoxide in the nitrogen flow is not reached and hence shows a decline in rate. At lower flow rates the reaction takes a longer time to establish a steady-state concentration gradient around the acid particle, shown by the plateau of the curve. This steady-state is reached in shorter time duration when higher flow rate $(<=2$ LPM $)$ is used. 


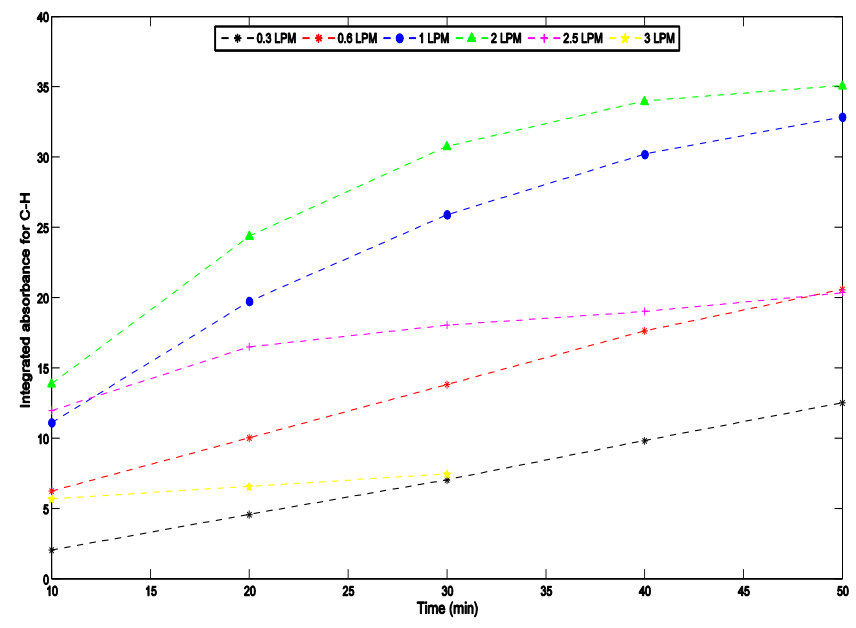

(a)

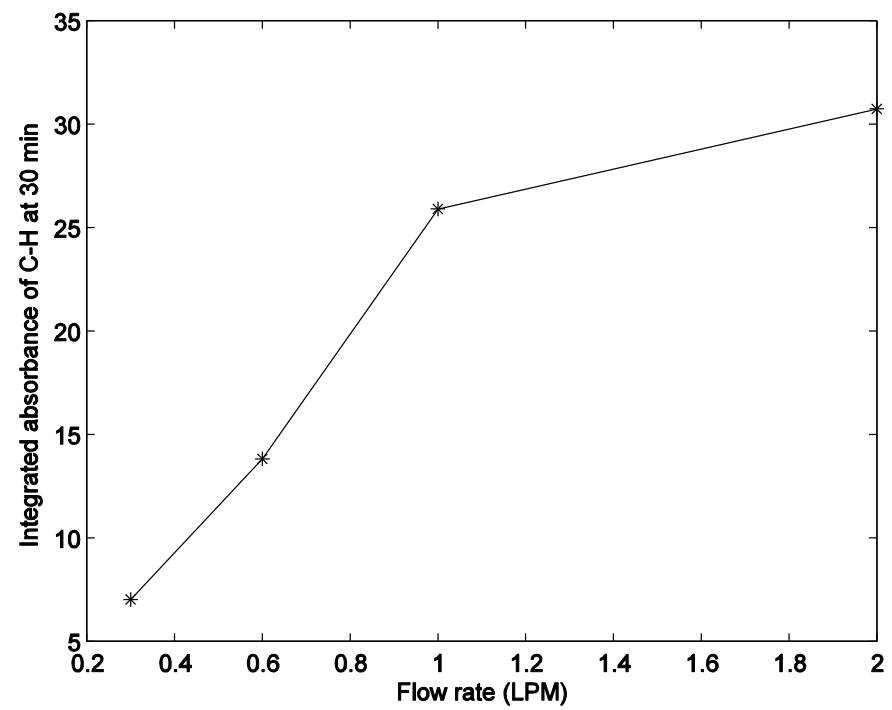

(b)

Figure 11: (a) Integrated absorbance of C-H $\left(3000-2850 \mathrm{~cm}^{-1}\right)$ peak of $\alpha$-pinene oxide reaction with sulfuric acid over time for different flow rates. (b) Integrated absorbance of C-H (3000$2850 \mathrm{~cm}^{-1}$ ) peak of $\alpha$-pinene oxide reaction with sulfuric acid after $30 \mathrm{~min}$ at different flow rates. 
Experiments employing different epoxide concentrations were also carried out both at $42 \%$ and $9 \% \mathrm{RH}$ conditions. Figure 12 shows the absorbance of the alkyl peak at different epoxide concentrations for a given reaction run-time. In each of the experiments absorption peaks are identified at similar frequencies showing that same products are formed. The overall intensity for all peaks was proportional to the epoxide concentration, which showed that the amount of organics formed depended on the epoxide concentration. This was evident by the increase and decrease in the absorbance intensities of all peaks with an increase and decrease in epoxide concentration, respectively. At lower epoxide concentrations the peak intensities decrease due to less formation of organics in the particle. This observation is consistent with the first order reaction rate of acid hydrolysis of epoxides with respect to epoxide concentration. At higher epoxide concentrations, the reaction was probably limited by the amount of $\mathrm{H}_{2} \mathrm{SO}_{4}$ or the equilibrium state of the reaction.

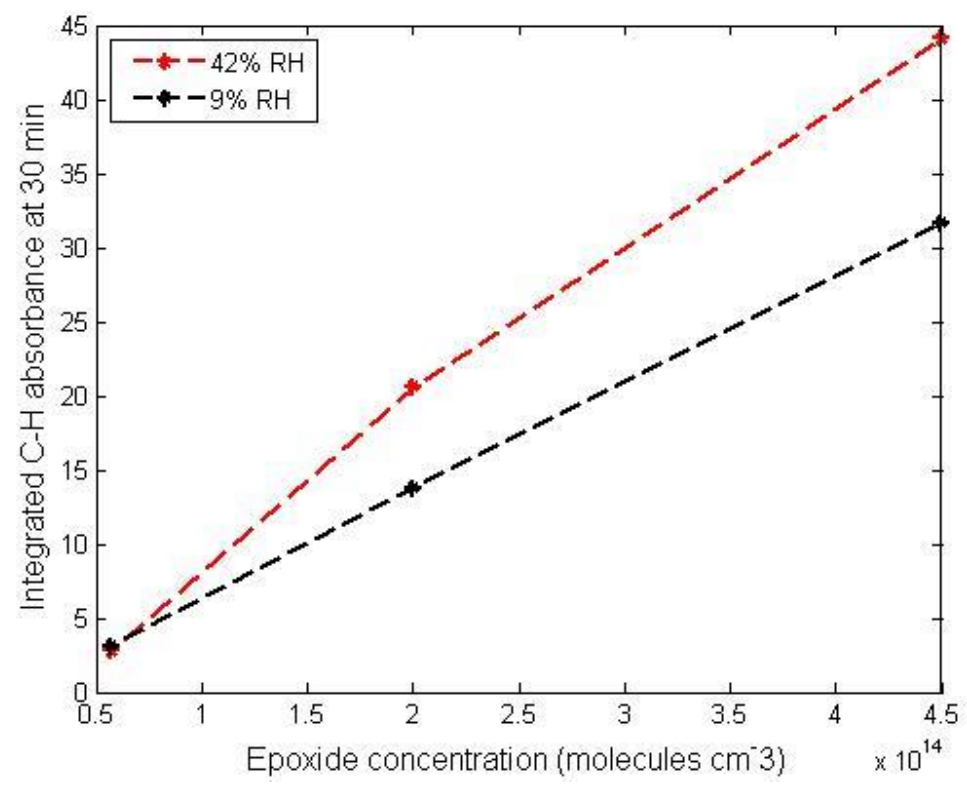

Figure 12: Integrated absorbance for C-H $\left(3000-2850 \mathrm{~cm}^{-1}\right)$ peak of $\alpha$-pinene oxide reaction with $\mathrm{H}_{2} \mathrm{SO}_{4}$ after $30 \mathrm{~min}$ for different epoxide concentrations at two RH conditions. 
$\alpha$-Pinene oxide reactions with ammonium sulfate and bisulfate were carried out to examine the importance of acid catalysis in hydrolysis reaction of epoxides.

Figure 23 in Appendix shows the IR spectra of ammonium sulfate and bisulfate at different RH. The IR spectrum of the reaction of $\alpha$-pinene oxide with ammonium bisulfate at $60 \% \mathrm{RH}$ is shown in Figure 13. Ammonium sulfate represents a close to neutral substrate used to study the epoxide reaction in comparison to the acidic surface. Reactions were carried out both with dry and deliquesced ammonium sulfate. The reaction was found to be very slow and therefore no identifiable products were formed in either case. This result is supported by observations in other studies at high pH [Minerath and Elrod, 2009]. However, with ammonium bisulfate, products are formed which have similar IR spectra as obtained at $84 \%$ RH conditions. This indicates formation of same products (diols) in the presence of small amount of acid.

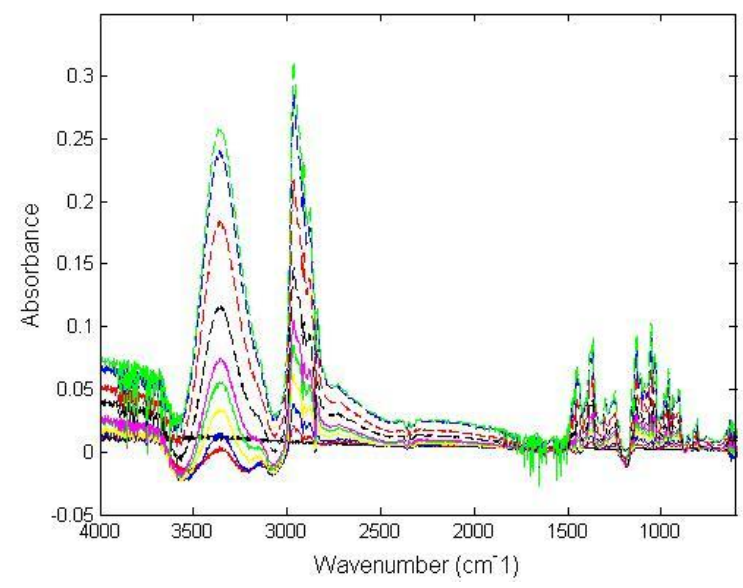

Figure 13: ATR-FTIR spectra of reaction of $\alpha$-pinene oxide with deliquesced ammonium bisulfate. 


\subsubsection{Isoprene oxide}

Experiments with isoprene oxide were carried out under conditions of different $\mathrm{RH}$ and epoxide concentrations. Figure 14 and Figure 15 show the ATR-FTIR absorbance for isoprene oxide reaction with sulfuric acid at different $\mathrm{RH}$. Detailed peak identification for the fingerprint region is provided in Table 7. From the $\alpha$-pinene oxide experiments at different flow rates, 1 LPM flow rate was found to be sufficient to overcome the diffusion limitation, so all the experiments for isoprene oxide were carried out at $\mathrm{T}=296 \pm 2 \mathrm{~K}$ and total flow rate of 1 LPM.

Table 7: ATR-FTIR absorption frequencies assigned to the functional groups formed by acid hydrolysis of isoprene oxide

\begin{tabular}{llll}
\hline Assignment & \multicolumn{3}{c}{ Frequencies $\left(\mathbf{c m}^{-1}\right)$} \\
of frequency & RH 80\% & RH 41\% & RH 14\% \\
\hline O-H stretch & 3372 & 3372 & 3460 \\
C-O stretch (primary alcohol/ ${ }^{*}$ acetal) & 1057 & 1058 & $1085^{*}$ \\
O-H in-plane deformation & 1250 & 1262 & 1278 \\
C-O stretch (tertiary alcohols/ ${ }^{*}$ acetal) & 1205 & 1202 & $1193^{*}$ \\
C-O deformation for tertiary alcohol & 784 & 787 & 792 \\
\hline
\end{tabular}




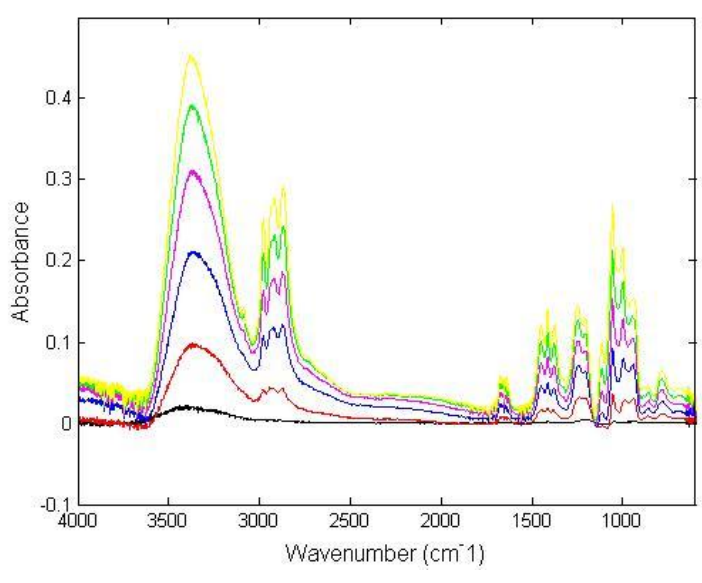

(a)

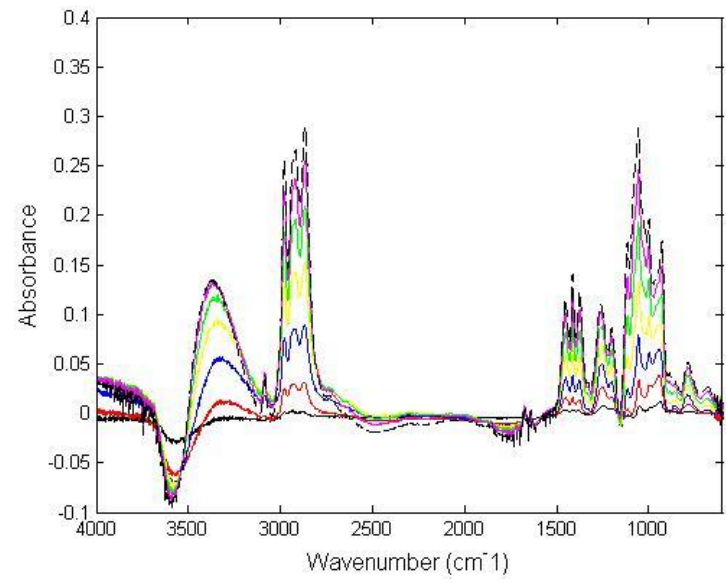

(b)

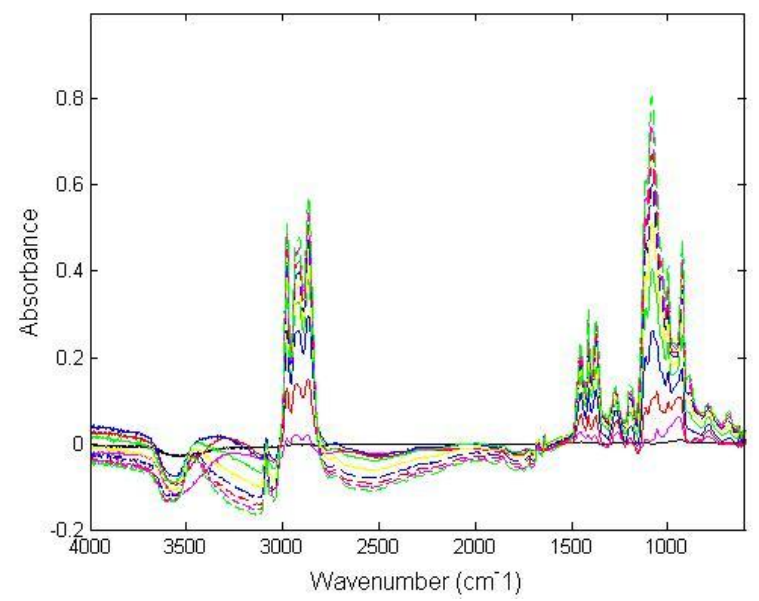

(c)

Figure 14: ATR-FTIR spectra of isoprene oxide reaction with sulfuric acid at (a) $80 \%$ RH (b) $41 \% \mathrm{RH}$ (c) $13 \% \mathrm{RH}$. Total time for the reactions was $30 \mathrm{~min}$ for the $\mathrm{RH}<=41 \%$ and $45 \mathrm{~min}$ for low RH. 


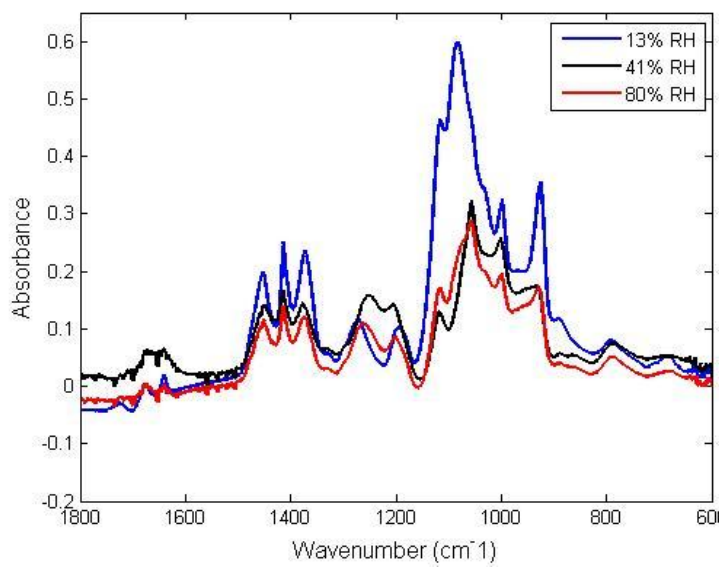

(a)

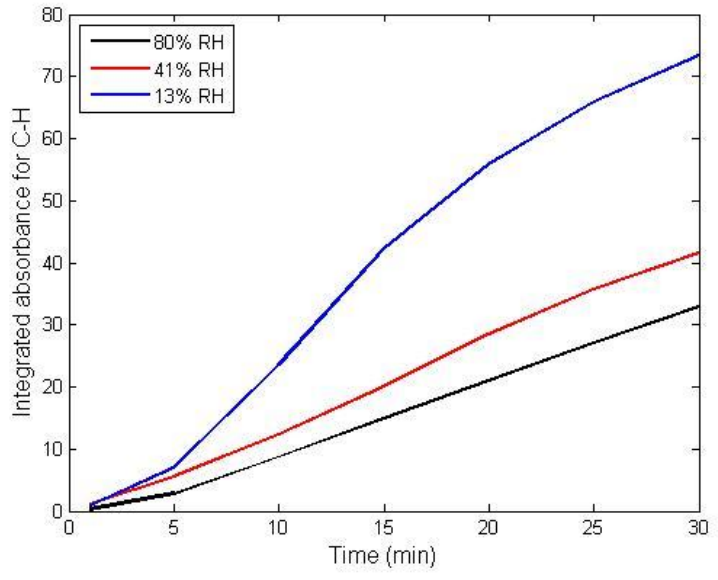

(b)

Figure 15: (a) IR spectra in the fingerprint region for the product of acid-epoxide reaction (for isoprene epoxide) under different humidity conditions (b) Integrated C-H absorbance over time for isoprene oxide at different RH. 
The peaks in the region $2978 \mathrm{~cm}^{-1}$ are assigned to the $\mathrm{C}-\mathrm{H}$ stretch of $-\mathrm{CH}_{3}$ group and peaks ranging between $2919-2913 \mathrm{~cm}^{-1}$ and $2868-2866 \mathrm{~cm}^{-1}$ correspond to the $-\mathrm{CH}_{2}$ asymmetric and symmetric stretch, respectively. The medium sharp peaks at $\sim 3090 \mathrm{~cm}^{-1}$ and peaks in the range $1000-925 \mathrm{~cm}^{-1}$ confirms presence of the vinyl group. This is also supported by the presence of peak in the $1680 \mathrm{~cm}^{-1}$ range showing the $\mathrm{C}=\mathrm{C}$ stretch. A small overtone of the vinylic stretch was observed in the $1800 \mathrm{~cm}^{-1}$ range. The peak at $3460 \mathrm{~cm}^{-1}$ observed at $13 \%$ $\mathrm{RH}$ represents the $\mathrm{O}-\mathrm{H}$ stretch. This band is concentration dependent and the frequency lowers in dilute solution due to increase in hydrogen bonding. Hence, the $\mathrm{O}-\mathrm{H}$ stretch peak is at $3372 \mathrm{~cm}^{-1}$ in case of $41 \% \mathrm{RH}$ and $3370 \mathrm{~cm}^{-1}$ at $80 \% \mathrm{RH}$. However, there are differences in the intensities and position of peaks for the low and high $\mathrm{RH}$ conditions. While the $\mathrm{O}-\mathrm{H}$ stretch vibration intensity in the range $3300-3500 \mathrm{~cm}^{-1}$ decreases with decreasing humidity, the intensity of peaks in the region of C-O stretch $\left(1200-1000 \mathrm{~cm}^{-1}\right)$ increases. This can be explained by the formation of different products at lower RH. For $80 \% \mathrm{RH}$ conditions, strong peaks for alcohol C-O stretching vibrations are observed in the region $1200-1000 \mathrm{~cm}^{-1}$. These frequencies are typical for the tertiary alcohol group. The C-O deformation of tertiary alcohols is assigned to the peaks $\sim 790 \mathrm{~cm}^{-1}$. The frequencies show up on the higher end due to presence of a $-\mathrm{CH}_{3}$ group attached to the carbon of $\mathrm{C}-\mathrm{OH}$. Strong peaks are observed at 1058 and $1057 \mathrm{~cm}^{-1}$ for the higher $\mathrm{RH}$ conditions, representing $\mathrm{C}-\mathrm{O}$ stretch for primary alcohol group. Similar observation is made at $41 \% \mathrm{RH}$. At $13 \% \mathrm{RH}$, the peak shifts to a higher frequency and shows up as a very strong peak at $1085 \mathrm{~cm}^{-1}$. This represents the strong band for $\mathrm{C}-\mathrm{O}-\mathrm{C}$ stretching vibration of acetals. Formation of acetals and increase in carbon chain is also evident by the increased intensities of all the alkyl $(\mathrm{C}-\mathrm{H})$ vibrations. The strong peaks in the region $1280-1250 \mathrm{~cm}^{-1}$ represent the $\mathrm{O}-\mathrm{H}$ in-plane deformation. The intensity of 
these peaks is seen to decrease with decreasing humidity. For high RH conditions, absorbance peaks for both primary and tertiary alcohol groups were identified. Hence the structure of the diol formed was confirmed to be 1,2-hydroxy isoprene. However, presence of any other diol is difficult to identify just from the ATR-FTIR spectrum. With decreasing humidity, and therefore, increasing acidity, acetal formation takes place which leads to increased absorbance intensities for the $\mathrm{C}-\mathrm{H}$ and $\mathrm{C}-\mathrm{O}-\mathrm{C}$ stretches and decreased $\mathrm{O}-\mathrm{H}$ vibrations. The overall intensity of the peaks increased with decreasing RH. The temporal evolution of the products as a function of RH is shown in Figure 15. The trend was found to be opposite to that of $\alpha$-pinene oxide. This observation can be attributed to the type of products formed for the two epoxides. Unlike for $\alpha$-pinene oxide, where organosulfates are formed at low RH, acetal formation takes place for isoprene oxide. The increase in carbon chain due to acetal formation leads to increased absorbance for the peaks. This acetal formation is enhanced with increasing acidity and hence leads to a rapid increase in absorbance.

Experiments for different epoxide concentrations were carried out at $13 \% \mathrm{RH}$ conditions only, since low volatile products were seen to form only at high acidity. At a particular acid concentration, the amount of product formed is seen to be proportional to the epoxide concentration, similar to that observed for $\alpha$-pinene oxide. Figure 16 shows the absorbance variation for different epoxide concentrations at $13 \% \mathrm{RH}$. 


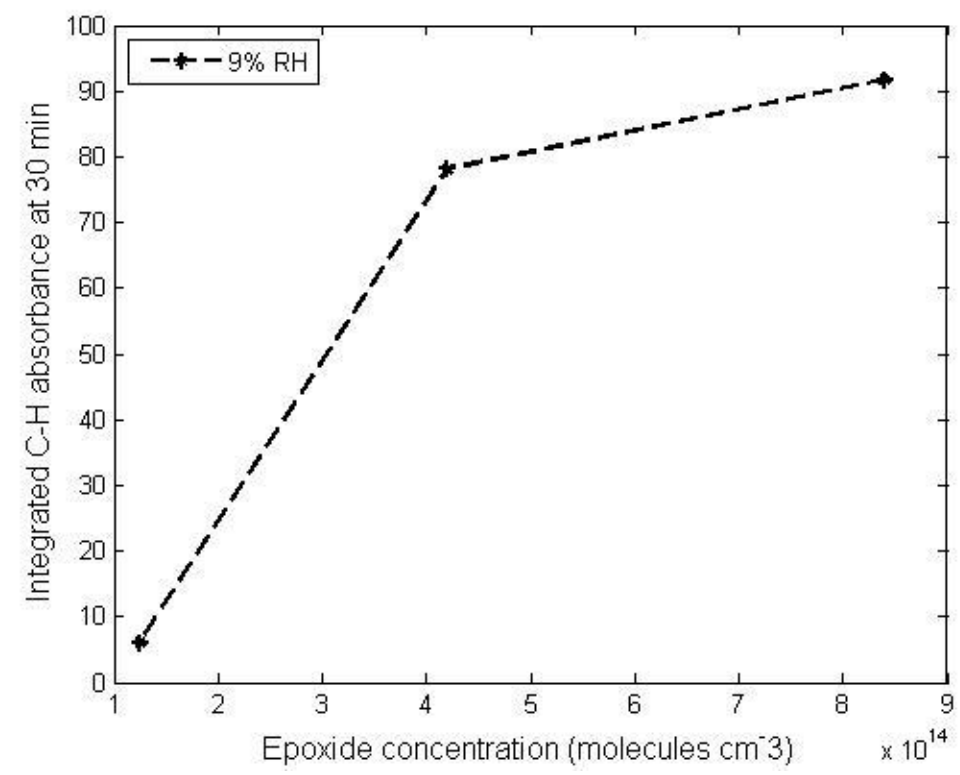

Figure 16: Integrated absorbance for $\mathrm{C}-\mathrm{H}\left(3000-2850 \mathrm{~cm}^{-1}\right)$ peak of isoprene oxide reaction with $\mathrm{H}_{2} \mathrm{SO}_{4}$ after 30 min for different epoxide concentrations at $13 \% \mathrm{RH}$.

Reactions with ammonium sulfate and bisulfate were carried out similarly as that with sulfuric acid. With both dry and deliquesced ammonium sulfate, the reaction was very slow to allow product formation, thus showing the importance of acid catalysis. The high hydrolysis rate constant reflects the stability of the intermediate carbocation for isoprene oxide [Minerath et al., 2009], and hence explains the slow reaction with ammonium sulfate (high $\mathrm{pH}$ ). In the presence of ammonium bisulfate, an absorption spectrum similar to that obtained at high $\mathrm{RH}$ conditions was obtained, indicating similar products being formed in both cases. 


\subsection{NMR Measurements}

\subsection{1 $\quad \alpha$-Pinene oxide}

NMR measurements were done to gain an insight on the products formed by reaction of epoxide with different concentrations of $\mathrm{D}_{2} \mathrm{SO}_{4}$ in bulk and if the proportions of the acid and epoxide reacted together had any effect on the products. It was difficult to analyze the product from the ATR-FTIR experiments extracted using a suitable solvent because a very small amount of product was formed which was insufficient for the $300 \mathrm{MHz}$ spectrometer. Therefore, bulk experiments with calculated epoxide to acid ratios were performed to study the epoxide hydrolysis reaction. The solvent used was deuterium oxide $\left(\mathrm{D}_{2} \mathrm{O}\right)$. Since this was an acid hydrolysis reaction, a shift in the HDO peak was expected in all the reactions with different acid concentrations. Hence, considering this peak unreliable, the protons of the geminal methyl groups of the $\alpha$-pinene oxide were taken as a reference, assuming that they are least affected by the change in chemical environment. One experiment was carried out for $\alpha$-pinene oxide reaction with $\mathrm{D}_{2} \mathrm{SO}_{4}(0.1 \% \mathrm{wt})$ and $\mathrm{Na}_{2} \mathrm{SO}_{4}$ solution (5\% wt) similar to the concentrations used in previous studies on acid hydrolysis of epoxides [Minerath and Elrod, 2009]. The chemical shifts thus obtained helped in estimating the position of sulfate peak. The result is shown in Figure 24 in Appendix. Both diol and sulfate ester formation was observed. A peak at $3.9 \mathrm{ppm}$ shows formation of diol and the peak at $5.5 \mathrm{ppm}$ shows formation of sulfate ester product. The integrated peak areas of the products reveal that they are formed in similar proportions. With comparison to other results, the sulfate group is found to be attached to the secondary carbon involved in epoxide ring, consistent with other studies [Iinuma et al., 2009]. Other sets of experiments were carried out without using $\mathrm{Na}_{2} \mathrm{SO}_{4}$. For the first set, $5 \mu \mathrm{l}$ of $\alpha$-pinene oxide was reacted with $5 \mathrm{ml}$ each of $5 \% \mathrm{wt}, 40 \%$ 
wt and $75 \%$ wt $\mathrm{D}_{2} \mathrm{SO}_{4}$ acid solutions prepared in $\mathrm{D}_{2} \mathrm{O}$. For acid concentrations above $5 \%$ wt, a dark brown solution was obtained on adding epoxide to acid with generation of heat. Organosulfate was possibly formed. However, due to presence of large amount of acid, some polymerization might have taken place which along with lower sensitivity of the spectrometer, made it difficult to obtain a good spectrum for the highly acidic solutions. Hence, only the spectra for the dilute concentrations have been reported. For 5\% wt acid, the reaction showed formation of diol and hydroxy-sulfate disulfate products (Appendix-Figure 25). A small peak representing double bond formation was also observed, showing some dehydration. Peaks at 3.8-4.0 ppm were assigned to protons attached to carbon with hydroxyl groups and peaks at 5.4-5.6 ppm were assigned to protons attached to carbon with sulfate groups. Two peaks, one at $3.8 \mathrm{ppm}$ represented protons of the diol product, while that at 4.0 ppm represented protons of hydroxy-sulfate, with sulfate attached to the tertiary carbon atom. The protons related to the sulfate group show upfield compared to those corresponding to the hydroxyl groups [Eddingsaas et al., 2010; Minerath and Elrod, 2009; Minerath et al., 2009]. The peak at $5.5 \mathrm{ppm}$ was assigned to the protons associated with the secondary carbon atom to which sulfate group is attached. The peak for disulfate product was seen at $5.6 \mathrm{ppm}$. In experiments with very dilute concentration of acids both diol and hydroxyl sulfate products were seen to form (Figure 26, Figure 27, Figure 28), but in different ratios. No dehydration products were observed in this case. This result is consistent with other studies on acid hydrolysis of epoxides [Iinuma et al., 2009; Minerath and Elrod, 2009]. Figure 17 shows ${ }^{1} \mathrm{H}$ NMR spectrum of reaction products of $\alpha$-pinene oxide with $0.7 \%$ wt $\mathrm{D}_{2} \mathrm{SO}_{4}$ in the epoxide to acid ratio of 1:63. In a third experiment using 1:10 ratio of epoxide to acid and $5 \% \mathrm{wt} \mathrm{D}_{2} \mathrm{SO}_{4}$, aldehyde product was also seen to form with a chemical shift at $9.6 \mathrm{ppm}$, along with diol and 
the sulfate ester (Appendix-Figure 29). This aldehyde could form as a result of rearrangement of $\alpha$-pinene oxide in presence of acid, as shown in a previous study [Iinuma et al., 2009]. Aldehyde was also formed in small amounts when the epoxide hydrolysis product with 5\% wt $\mathrm{D}_{2} \mathrm{SO}_{4}$ was diluted 10 times with $\mathrm{D}_{2} \mathrm{O}$ (Appendix-Figure 30). The relative proportions of products formed in each case are shown in Figure 18. Diol was found to be one of the major products in all cases. The sulfate product yield increased with increasing acid concentration. Also, an increase in acidity led to different branching ratios of the sulfate products. For very dilute concentrations, the 2-pinanol-3-hydrogen sulfate product was preferably formed, with the sulfate group attaching to the less sterically hindered carbon atom. This product forms by esterification of diol produced at the first step by hydrolysis of epoxide. With increasing acidity, the polar and hyperconjugative effects of the methyl group also start playing a role thus overcoming the steric effects and lead to more formation of hydroxy-sulfate with sulfate group attached to the tertiary carbon atom [Parker and Isaacs, 1959]. Hence, the ratio of 3pinanol-2-hydrogen sulfate to 3-pinanol-2-hydrogen sulfate is seen to increase with increasing acidity. Also, with increasing acidity and hence molar concentration of sulfate, diester products are seen to form. Earlier studies have shown that the yield of sulfate ester formed by acid catalyzed reactions of epoxides depends on sulfate concentration and not on $\mathrm{H}^{+}$(or $\mathrm{HSO}_{4}^{-}$) [Eddingsaas et al., 2010; Minerath and Elrod, 2009; Minerath et al., 2009]. The observations in this study are consistent with other studies in showing dependency on available sulfate concentration (Figure 19). With respect to diol formation, the NMR results obtained in this study are in agreement with the ATR-FTIR results. Formation of organosulfate in NMR studies with 5\% wt acid and absence of any organosulfate at low acid concentration in ATR-FTIR studies can be explained by the kinetic isotope effect (KIE). KIE 
represents the ratio of the isotopic rate constants $\left(k_{H} / k_{D}\right)$ that is known to play a significant role in several chemical reactions [Limbach, 1991]. The theory behind KIE states that for a rate determining step of a chemical reaction, the isotope substitution involved in a bond formation or cleavage greatly modifies the reaction rate. The major contribution to this effect is due to the change in the stretching and bending vibrational frequency of the C-H or C-D bond. The isotope effect for the hydrogen-deuterium replacements in particular is very large due to the exclusive mass relation between the two isotopes. Also, KIE has been found to be very large for SN1 reactions as compared to the SN2 reactions, because of the nature of the transition state involved [Van Doorslaer et al., 1984]. In the acid hydrolysis reaction of epoxides, the protonation of the epoxide oxygen and breaking of the $\mathrm{C}-\mathrm{O}$ bond leading to formation of a carbocation is the rate determining step. Presence of deuterium, both from solvent and the reactant, can lead to large KIE for this step. Also, the different products formed from this common intermediate are likely to have different individual KIE. The competition between these product formation pathways can lead to an overall enhanced KIE for the complete reaction. This has been explained in previous studies [Thibblin, 1988; Thibblin and Ahlberg, 1989]. Also, since the isotope effect on the protonation of epoxide is very large in the presence of deuterated solvent, this could lead to an extremely high total KIE for the reaction [Thibblin, 1988; Thibblin and Ahlberg, 1989]. The KIE values for reactions reported so far range from $<1$ to $\sim 200$, depending on the nature of reaction and temperature [Limbach, 1991]. Tunneling effect, a quantum mechanical phenomenon, might also play a role in modifying reaction rates. This phenomenon accounts for the wave-like nature of the hydrogen atom and explains the skipping of energy barriers by the hydrogen 
atom, thus increasing the rate of reaction. Both KIE and tunneling effects are pronounced at lower temperatures [Limbach, 1991; Wiberg, 1955].

Use of $\mathrm{D}_{2} \mathrm{O}$ as solvent plays an important role in the acid catalyzed hydrolysis reaction [Wiberg, 1955]. From the difference in products formed in experiment 4 and 6 as shown in Figure 18 , it is seen that the reaction proceeds slowly in the presence of excess $\mathrm{D}_{2} \mathrm{O}$, leading to aldehyde formation and less diol formation.

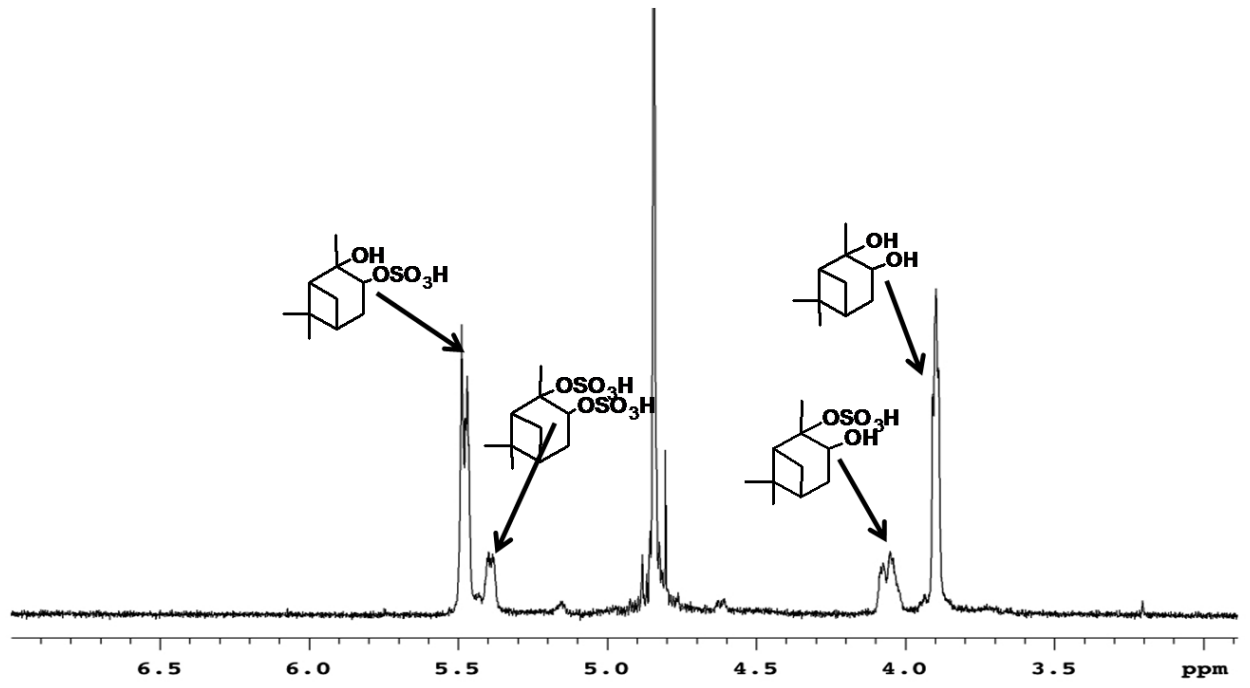

Figure 17: ${ }^{1} \mathrm{H}$ NMR spectrum of $\alpha$-pinene oxide reaction with $0.7 \%$ wt $\mathrm{D}_{2} \mathrm{SO}_{4}$ at $298 \mathrm{~K}$. The chemical shifts depicted are associated with the protons attached to carbons with the respective functional groups. 


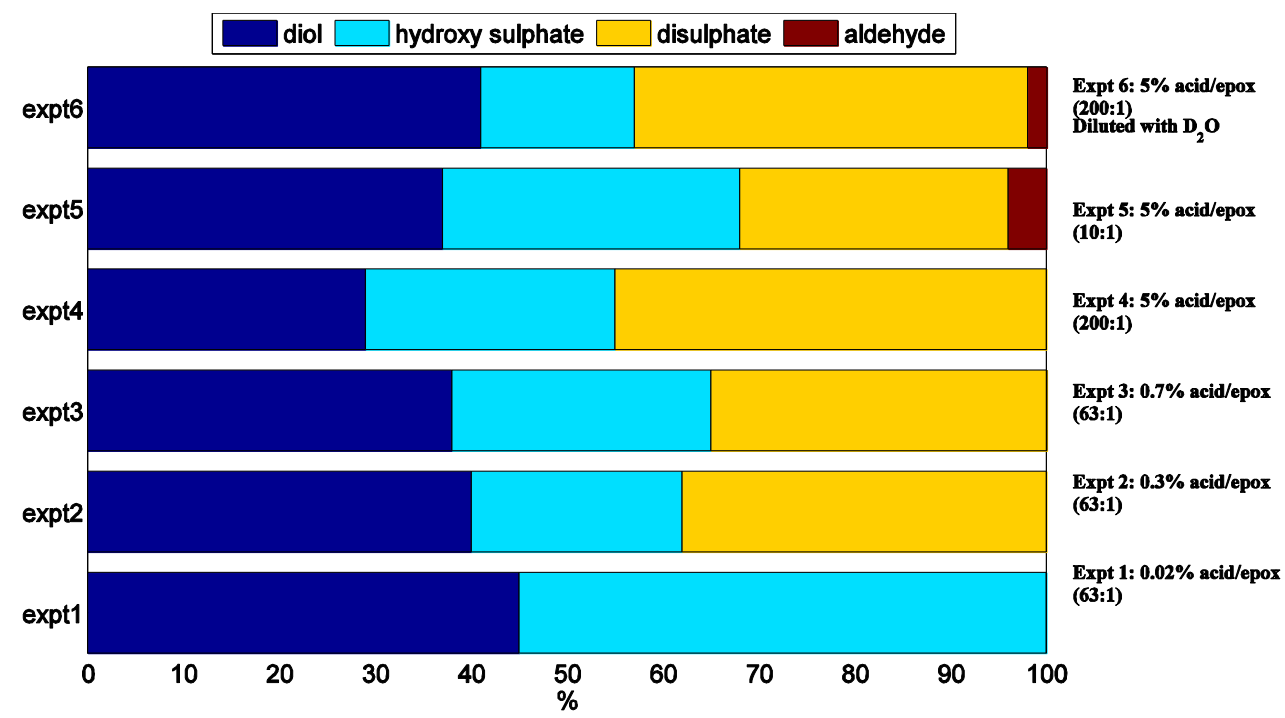

Figure 18: Relative proportions of products formed in different experiments of $\alpha$-pinene oxide with deuterated sulfuric acid.

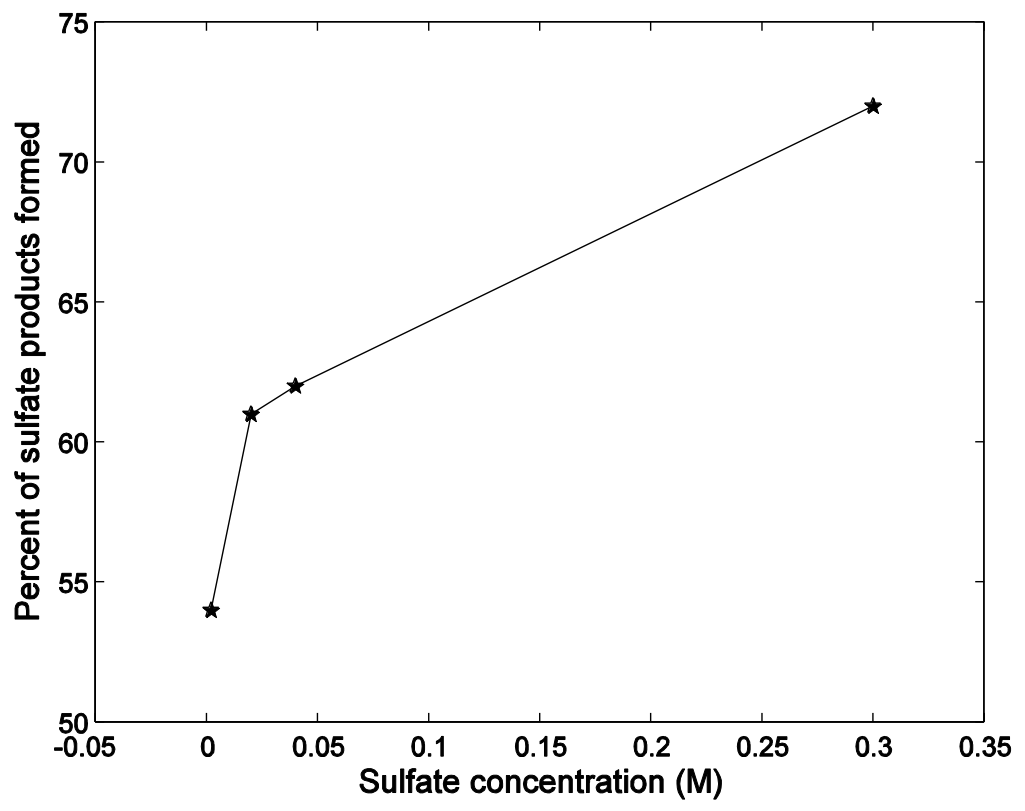

Figure 19: Sulfate ester product yield as a function of available acidic sulfate in solution. 


\subsubsection{Isoprene oxide}

For isoprene oxide, 3 experiments were carried out for product identification using different sulfuric acid concentrations. In all the experiments, the peaks were referenced to the protons of the methyl group attached to the $\mathrm{C}-2$ carbon of the isoprene oxide molecule, which were assumed to be least affected by a change in chemical environment. For the experiment with $5 \%$ wt $\mathrm{D}_{2} \mathrm{SO}_{4} / \mathrm{D}_{2} \mathrm{O}$, a mixture of products was identified including 1,2 and 1,4-diols and aldehyde (Appendix-Figure 31). Unlike previous studies [Minerath et al., 2009], where only 1,2-diol was reported, in this study, considerable amounts of 1,4-diol and aldehyde were also observed. However, similar to other studies, no organosulfate formation was observed [Minerath et al., 2009]. A tertiary carbocation was formed by ring opening of epoxide and another $\mathrm{OH}$ group attached to this carbon. For the 1,4-diol product, an allylic rearrangement was observed, confirmed by the peaks $\sim 4 \mathrm{ppm}$, which represent the protons attached to carbons containing $\mathrm{OH}$ groups, and the peak at $5.3 \mathrm{ppm}$ representing hydrogen of the double bond. Literature studies show that epoxides form aldehydes in the presence of acid [Parker and Isaacs, 1959]. A small peak at $9.3 \mathrm{ppm}$ is assigned to aldehyde group. Also, a peak is observed around $7 \mathrm{ppm}$ which represents a proton attached to the double bonded carbon atom adjacent to the aldehyde group. This shows that an aldehyde group conjugated to double bond is a favored structure.

In other experiments with isoprene oxide, similar products were observed. At very low acid concentration $(0.7 \% \mathrm{wt})$, aldehyde formation is negligible (Appendix-Figure 32). At higher epoxide to acid ratio, the ratios of the products change, but overall the products are the same (Appendix-Figure 33). The ratios of products for all the experiments are shown in Figure 20. The observation of aldehydes and no acetal formation in the NMR bulk studies can partially 
be explained by KIE. KIE accounts for the slower reaction in $\mathrm{D}_{2} \mathrm{O} / \mathrm{D}_{2} \mathrm{SO}_{4}$. Therefore, no aldehydes observed in ATR-FTIR studies can be attributed to faster reaction with $\mathrm{H}_{2} \mathrm{O} / \mathrm{H}_{2} \mathrm{SO}_{4}$. Also, for NMR studies where aldehydes are observed at low acid concentrations, the concentration might not be enough to initiate any acetal formation. However, acetal formation takes place at higher acidity conditions in the ATR-FTIR experiments.

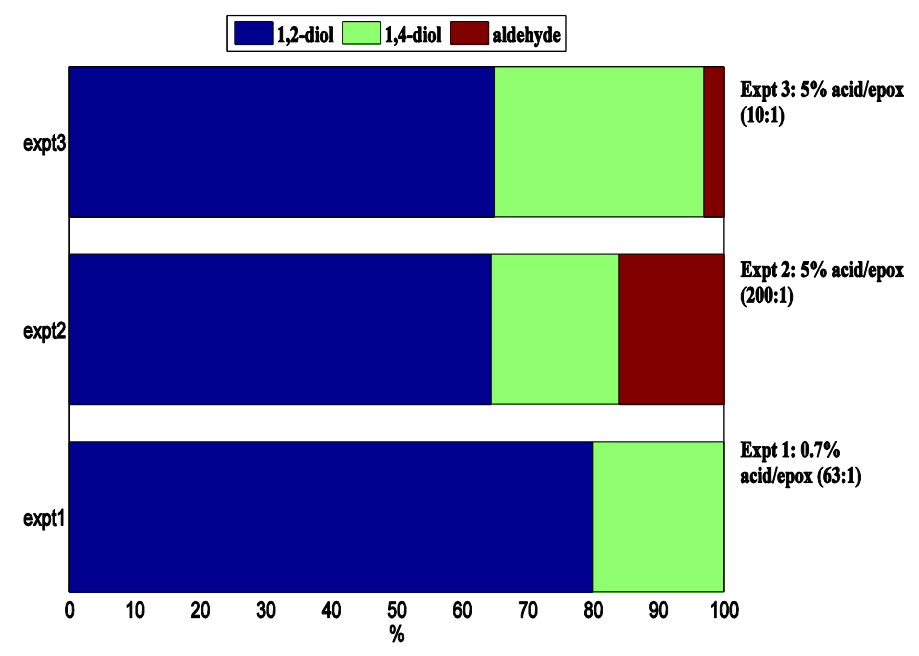

Figure 20: Relative proportions of products formed in different experiments of isoprene oxide with deuterated sulfuric acid.

In contrast to the observations made in the study by [Eddingsaas et al., 2010], where sulfate ester was detected in the reaction of cis-2,3-epoxybutane-1,4-diol with $1 \mathrm{M} \mathrm{H}_{2} \mathrm{SO}_{4}$ solution, no sulfate ester was formed in case of isoprene epoxide. This can be explained by the extremely high acid hydrolysis reaction rate coefficient of isoprene epoxide measured by [Minerath et al., 2009]. In epoxides with $\mathrm{OH}$ substituents, the reactivity is reduced. The presence of hydroxyl group stabilizes the carbocation and makes it more favorable to the nucleophilic attack. The same reason explains the observance of sulfate esters in case of $\alpha$ - 
pinene oxide reaction with sulfuric acid. Due to smaller hydrolysis rate coefficient of $\alpha$ pinene oxide, the $\mathrm{HSO}_{4}{ }^{-}$nucleophile is able to attack the ring. In our case, we observed aldehyde formation from epoxide, which has been observed for epoxides in presence of acids [Parker and Isaacs, 1959]. Protonation of the epoxide oxygen leads to formation of a conjugate acid of the epoxide which is the equilibrium state. The reaction of this intermediate is the rate determining step and depending on the conditions, can undergo a SN1 or a modified SN2 mechanism [Darer et al., 2011; Parker and Isaacs, 1959]. The stability of this conjugate intermediate (carbocation) is the key to formation of different products, since it is more reactive than the reactant epoxide. Figure 21 shows ${ }^{1} \mathrm{H}$ NMR spectrum of reaction products of isoprene oxide with $5 \%$ wt $\mathrm{D}_{2} \mathrm{SO}_{4}$ in the epoxide to acid ratio of 1:200.

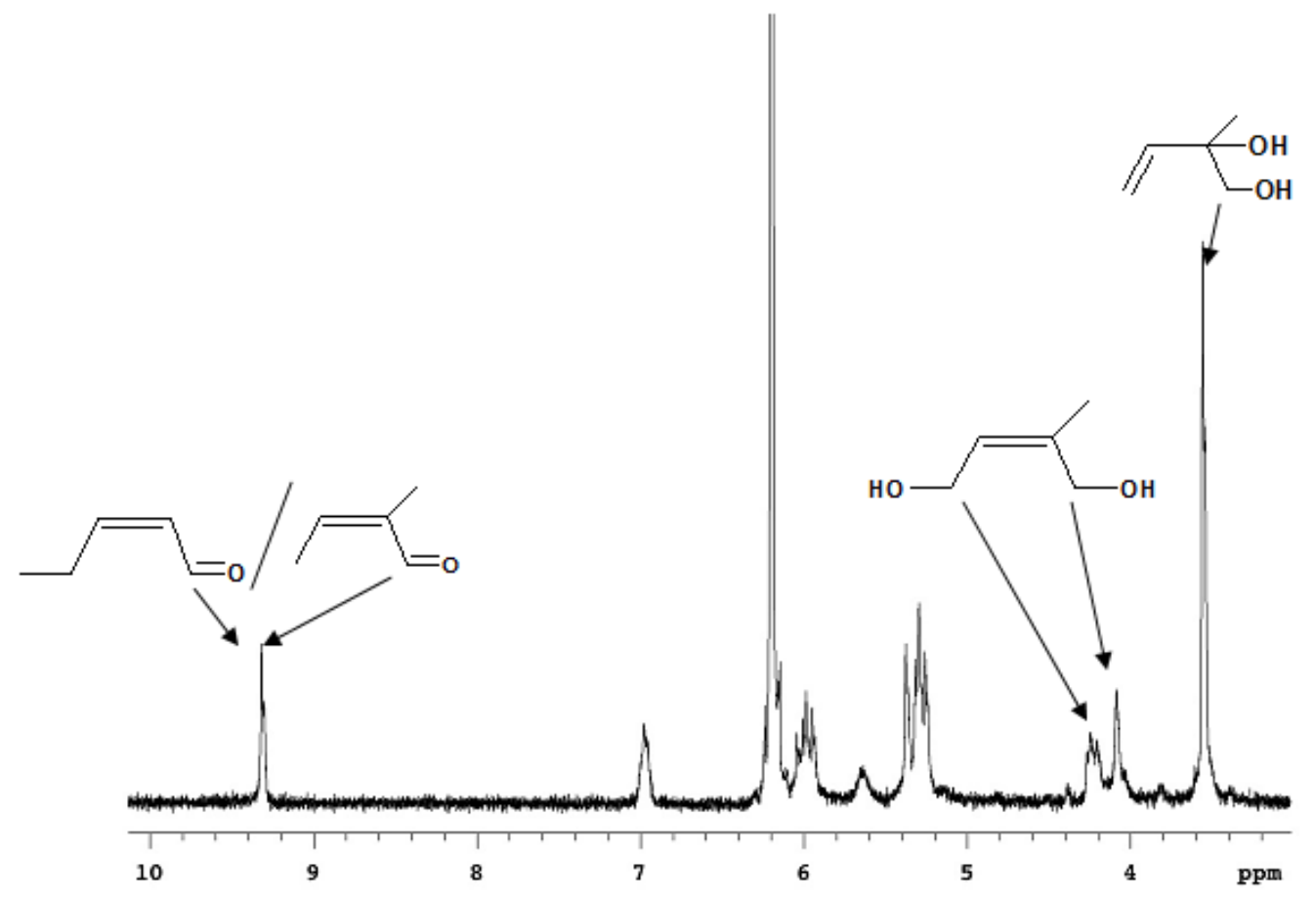

Figure 21: ${ }^{1} \mathrm{H}$ NMR spectrum of isoprene oxide reaction with $5 \%$ wt $\mathrm{D}_{2} \mathrm{SO}_{4}$ at $298 \mathrm{~K}$. The chemical shifts depicted are associated with the protons attached to carbons with the respective functional groups. 


\section{CONCLUSIONS}

This study presents an extensive analysis of the heterogeneous reaction of epoxides with sulfuric acid. Epoxides have been previously identified as important intermediate species in the oxidation of isoprene and other alkenes. The reaction of these epoxides with sulfuric acid was studied using different techniques in order to understand the reaction mechanism and the type of products formed under different conditions. Study of heterogeneous reaction between sulfuric acid and epoxide using low pressure laminar flow ID-CIMS and ATR-FTIR spectroscopy both revealed irreversible uptake of epoxide by sulfuric acid. In the uptake experiments using ID-CIMS, the reaction between epoxide and sulfuric acid was found to be temperature dependent. Room temperature experiments with high acid concentration showed an irreversible uptake of epoxide leading to formation of less volatile products. These products, based on literature studies and observations from other methods could be diols, sulfate esters and condensation products like aldols and acetals. At lower temperatures, the formation of some other more volatile species with same $\mathrm{m} / \mathrm{z}$ as epoxide was seen to be preferred over the formation of the less volatile products. The more volatile products were interpreted to be mostly hydroxy-alkenes and aldehydes. These volatile organics overlapped with the parent epoxide signal thus making it difficult to obtain uptake coefficients at lower temperatures. At room temperature, the uptake coefficient for isoprene oxide over $96 \% \mathrm{wt}$ acid was an order higher than that for $\alpha$-pinene oxide. This is evident from the high acid catalyzed hydrolysis reaction rate constant measured for isoprene oxide [Minerath et al., 2009]. 
The ATR-FTIR studies were performed to gain an insight on the types of products formed for reaction of epoxides with different sulfuric acid concentrations. A high RH condition implied low acid concentration and similarly a low RH condition implied high acidity. Experiments with $\alpha$-pinene oxide revealed that at high humidity, diols were the major identifiable product. With decreasing humidity, the acid concentration increased and hence the amount of available acidic sulfate. At low RH conditions, formation of organosulfate was observed. This was also supported by the decrease in the absorbance by hydroxyl groups. The overall absorbance for all functional groups decreased with decreasing RH, which was explained with the help of optical microscopic images. More product formation and higher water activity at high RH increased wettability and hence contact with the ATR crystal. This led to an increase in reaction rate and higher absorbances. Loss of these hydroxyl groups with decreasing RH was attributed to esterification and successful attachment of sulfate group to the carbocation in competition with the hydroxyl group. The reaction between gaseous epoxide and sulfuric acid droplets was found to be diffusion limited at atmospheric pressure. The amount of organics formed in the particle phase increased with increasing epoxide concentration, irrespective of RH. The diffusion limitation and dependency on epoxide concentration was also applicable to the experiments with isoprene oxide. But the products obtained for isoprene oxide at different $\mathrm{RH}$ conditions and the reaction rates varied from those for $\alpha$-pinene oxide. At higher RH conditions, diols were the only products observed. There was a possibility of both 1,2-diol and 1,4-diol to form under high RH conditions. But at lower RH, acetal formation was observed, evident by the enhanced absorbance of C-O-C vibration typical for acetals. Increased absorbances for alkyl and C-O stretching vibrations, and reduced absorbance for hydroxyl groups indicated the increase in carbon chain by 
formation of high molecular weight oligomers. No organosulfate formation was observed for isoprene oxide in these ATR-FTIR studies, owing to its high hydrolysis rate constant.

The acid catalyzed hydrolysis reaction of epoxides was confirmed by the absence of any products in neutral environment, for the same reaction time. Ammonium sulfate was used as a neutral substrate for reaction. No products were identified even after a long reaction time ( $\sim 30 \mathrm{~min})$, irrespective of the dry or deliquesced state of ammonium sulfate. This suggested the importance of acid catalysis in the heterogeneous reactions of epoxides and the slow reaction of epoxide under neutral condition. Ammonium bisulfate represented an acidic environment similar to the high humidity conditions, and similar products were seen to form for both epoxides as observed at high RH.

NMR bulk studies revealed another aspect of epoxide reaction with acid where the reaction was purely in solution phase. These experiments showed that the products formed by heterogeneous reactions are different from those formed by reactions in solution phase. In NMR experiments for isoprene oxide, diols and aldehydes were observed at low acid concentration. Similarly for $\alpha$-pinene oxide, organosulfate formation was observed even at very dilute acid concentrations, and the amount of sulfate products formed was proportional to the available acidic sulfate. In ATR-FTIR experiments, aldehydes were not observed as such, though acetals were observed directly at high acid concentration. Organosulfate formation was also observed only at high acidities. This observation was explained by the kinetic isotopic effect, which accounts for slower reactions in the deuterated solvents $\left(\mathrm{D}_{2} \mathrm{SO}_{4} / \mathrm{D}_{2} \mathrm{O}\right)$ and allows formation of other products at low acid concentration. These results indicate that epoxides react with acid forming low volatile and high molecular weight products that can contribute to SOA formation and growth. The reaction mechanism and 
products formed depend on the acid concentration, amount of water in aerosol and temperature.

\subsection{Atmospheric implications}

From the laboratory measurements of the uptake coefficients and product analysis at different acidities and temperatures, we can extrapolate the results to ambient conditions. The uptake coefficient values suggest that the heterogeneous reaction between the epoxides and sulfuric acid is efficient and can contribute to SOA growth. Using the uptake coefficient values, the lifetime of isoprene oxide and $\alpha$-pinene oxide for a heterogeneous reaction can be calculated from the following relation:

$$
k=\frac{1}{4} \gamma \omega \mathrm{A}
$$

where $\mathrm{A}$ is the total surface area of aerosols and $\gamma$ is the upper limit value for uptake coefficient.

$$
\tau=\frac{1}{k}
$$

It can be estimated that for a sulfuric acid aerosol population with a total surface area of $5 \times 10^{2} \mu \mathrm{m}^{2} \mathrm{~cm}^{-3}$, the lifetime of both the isoprene epoxide is $\sim 15 \mathrm{~min}$ and of $\alpha$-pinene oxide is $\sim 1$ hour. The lifetime for a heterogeneous reaction can be compared to that for the gaseous oxidation by $\mathrm{OH}$ and $\mathrm{O}_{3}$. Taking the global concentration of $\mathrm{OH}\left(10^{6}\right.$ molecules $\left.\mathrm{cm}^{-3}\right)$ and $\mathrm{O}_{3}$ $\left(10^{12}\right.$ molecules $\left.\mathrm{cm}^{-3}\right)$ [Seinfeld and Pandis, 2006], and assuming a rate constant close to vinyl ethers [Al Mulla et al., 2010; Zhou et al., 2006], the lifetime of isoprene oxide against $\mathrm{OH}$ is found to be 3-6 hours and against $\mathrm{O}_{3}>1$ day. Similarly, for $\alpha$-pinene oxide lifetime against $\mathrm{OH}$ and $\mathrm{O}_{3}$ would be 1 day to several days assuming a rate constant similar to that of 
a cyclic ether 1,8-cineole [Atkinson and Arey, 2003; Atkinson et al., 1990; Corchnoy and Atkinson, 1990]. These estimates suggest that heterogeneous reactions on acidic aerosols would be faster and more efficient than gaseous reactions with atmospheric oxidants and hence will be the major removal pathway for epoxides. The growth rate can be calculated using the following equation for the kinetic regime (modified from [Seinfeld and Pandis, 2006])

$$
\frac{d R}{d t}=\frac{M \omega \gamma\left(c_{\infty}-c_{s}\right)}{4 \rho}
$$

where $c_{\infty}$ and $c_{s}$ are the concentrations of epoxide $\left(\right.$ molecules $\mathrm{cm}^{-3}$ ) far from the particle and at the particle surface, respectively, and $\mathrm{M}$ and $\rho$ are the molecular weight and the density of sulfuric acid aerosol. It is found that the growth rate for isoprene oxide is $>10 \mathrm{~nm} \mathrm{hr}^{-1}$ and for $\alpha$-pinene oxide is about $5 \mathrm{~nm} \mathrm{hr}^{-1}$. These growth rates and the lifetime estimates are inclusive of the uncertainties associated with the reaction rates considered and other ambient conditions like temperature and RH. However, the values indicate that under conditions for particles containing high amount of acid and at lower tropospheric temperatures, the reaction between epoxides can take place very efficiently and could lead to significant growth of particles. Ambient measurements have shown that in urban environment, particle acidity varies over a wide $\mathrm{pH}$ range of 0-5 [Q Zhang et al., 2007]. Epoxides can react effectively in this $\mathrm{pH}$ range to contribute to SOA growth. The products formed by the heterogeneous reaction of epoxide with sulfuric acid are low volatile products which could modify both the chemical and physical properties of the aerosols, thus affecting their radiative and cloud forming properties. The results obtained in this study regarding the contribution of epoxides 
to growth of sulfuric acid particles are supported by the ongoing work in our lab, wherein high growth rates have been obtained for the two epoxides with sulfuric acid nanoparticles.

This study has revealed a wide range of products that can form from acid hydrolysis of epoxides, several of them identified for the first time for the selected epoxides. These results can also be expected from other epoxides, thus proving that under certain conditions, epoxides can be very efficient SOA precursors. These conditions include urban atmospheres where higher temperatures and very high $\mathrm{SO}_{2}$ concentrations are expected. From these studies, it is clear that even the highly volatile isoprene oxide can contribute to SOA formation and growth very efficiently. However, further research is required to quantify these products and to obtain a better understanding of the reactivity of epoxides towards acids at lower temperatures. Other epoxides need to be studied in order to identify other organic products that could contribute to SOA growth. 


\section{REFERENCES}

ACD/PhysChem suite, version 10.00, Advanced Chemistry Development, Inc., Toronto, ON, Canada, www.acdlabs.com, 2006.

Al Mulla, I., L. Viera, R. Morris, H. Sidebottom, J. Treacy, et al. (2010), Kinetics and Mechanisms for the Reactions of Ozone with Unsaturated Oxygenated Compounds, Chemphyschem, 11(18), 4069-4078.

Alvarado, A., E. C. Tuazon, S. M. Aschmann, R. Atkinson, and J. Arey (1998), Products of the gas-phase reactions of $\mathrm{O}\left({ }^{3} \mathrm{P}\right)$ atoms and $\mathrm{O}_{3}$ with alpha-pinene and 1,2-dimethyl-1cyclohexene, J. Geophys. Res., 103(D19), 25541-25551.

Ambrose, J. L., K. Haase, R. S. Russo, Y. Zhou, M. L. White, et al. (2010), A comparison of GC-FID and PTR-MS toluene measurements in ambient air under conditions of enhanced monoterpene loading, Atmos. Meas. Tech., 3(4), 959-980.

Arneth, A., R. K. Monson, G. Schurgers, U. Niinemets, and P. I. Palmer (2008), Why are estimates of global terrestrial isoprene emissions so similar (and why is this not so for monoterpenes)?, Atmos. Chem. Phys., 8(16), 4605-4620.

Atkinson, R., and J. Arey (2003), Gas-phase tropospheric chemistry of biogenic volatile organic compounds: a review, Atmos. Env., 37(Supplement 2), 197-219.

Atkinson, R., D. Hasegawa, and S. M. Aschmann (1990), Rate constants for the gas-phase reactions of $\mathrm{O}_{3}$ with a series of monoterpenes and related compounds at $296 \pm 2 \mathrm{~K}$, Int. J. Chem. Kinet., 22(8), 871-887.

Atkinson, R., J. Arey, S. Aschmann, and E. Tuazon (1994a), Formation of O $\left({ }^{3} \mathrm{P}\right)$ atoms and epoxides from the gas- phase reaction of $\mathrm{O}_{3}$; with isoprene, Res. Chem. Intermediat., 20(3), 385-394.

Atkinson, R., S. M. Aschmann, J. Arey, and E. C. Tuazon (1994b), Formation yields of epoxides and $\mathrm{O}\left({ }^{3} \mathrm{P}\right)$ atoms from the gas-phase reactions of $\mathrm{O}_{3}$ with a series of alkenes, Int. J. Chem. Kinet., 26(9), 945-950.

Baumgardner, D., G. L. Kok, and G. B. Raga (2007), On the diurnal variability of particle properties related to light absorbing carbon in Mexico City, Atmos. Chem. Phys., 7(10), 2517-2526.

Benter, T., M. Liesner, R. N. Schindler, H. Skov, J. Hjorth, et al. (1994), Rempi-Ms and Ftir Study of $\mathrm{NO}_{2}$ and Oxirane Formation in the Reactions of Unsaturated-Hydrocarbons with $\mathrm{NO}_{3}$ Radicals, J. Phys. Chem-Us, 98(41), 10492-10496. 
Berndt, T., and O. Boge (1997a), Products and mechanism of the gas-phase reaction of $\mathrm{NO}_{3}$ radicals with alpha-pinene, J Chem Soc Faraday T, 93(17), 3021-3027.

Berndt, T., and O. Boge (1997b), Gas-phase reaction of $\mathrm{NO}_{3}$ radicals with isoprene: A kinetic and mechanistic study, Int. J. Chem. Kinet., 29(10), 755-765.

Blake, R. S., P. S. Monks, and A. M. Ellis (2009), Proton-Transfer Reaction Mass Spectrometry, Chem. Rev., 109(3), 861-896.

Bryan, W. P., and R. H. Byrne (1970), A calcium chloride solution, dry-ice, low temperature bath, J. Chem. Educ., 47(5), 361.

Carlton, A. G., C. Wiedinmyer, and J. H. Kroll (2009), A review of Secondary Organic Aerosol (SOA) formation from isoprene, Atmos. Chem. Phys., 9(14), 4987-5005.

Carr, G., G. Dosanjh, A. P. Millar, and D. Whittaker (1994), Ring opening of [a]-pinene epoxide, Journal of the Chemical Society, Perkin Transactions 2(7), 1419-1422.

Casale, M. T., A. R. Richman, M. J. Elrod, R. M. Garland, M. R. Beaver, et al. (2007), Kinetics of acid-catalyzed aldol condensation reactions of aliphatic aldehydes, Atmos. Environ., 41(29), 6212-6224.

Clark, D. J., and G. Williams (1957), Esterification by Sulphuric Acid .2. Ethyl Alcohol, J. Chem. Soc., 4218-4221.

Corchnoy, S. B., and R. Atkinson (1990), Kinetics of the gas-phase reactions of hydroxyl and nitrogen oxide $\left(\mathrm{NO}_{3}\right)$ radicals with 2-carene, 1,8-cineole, p-cymene, and terpinolene, Environ. Sci. Technol., 24(10), 1497-1502.

Darer, A. I., N. C. Cole-Filipiak, A. E. O’Connor, and M. J. Elrod (2011), Formation and Stability of Atmospherically Relevant Isoprene-Derived Organosulfates and Organonitrates, Environ. Sci. Technol., 45(5), 1895-1902.

Dhooghe, F., C. Amelynck, N. Schoon, E. Debie, P. Bultinck, et al. (2008), A selected ion flow tube study of the reactions of $\mathrm{H}_{3} \mathrm{O}^{+}, \mathrm{NO}^{+}$and $\mathrm{O}_{2}(+)$ with a series of sesquiterpenes, Int. J. Mass Spectrom., 272(2-3), 137-148.

Doyle, G. J. (1961), Self-Nucleation in Sulfuric Acid-Water System, J. Chem. Phys., 35(3), 795-799.

Eddingsaas, N. C., D. G. VanderVelde, and P. O. Wennberg (2010), Kinetics and Products of the Acid-Catalyzed Ring-Opening of Atmospherically Relevant Butyl Epoxy Alcohols, J. Phys. Chem. A, 114(31), 8106-8113. 
Fortner, E. C., J. Zhao, and R. Zhang (2004), Development of ion drift-chemical ionization mass spectrometry, Anal. Chem., 76(18), 5436-5440.

Fortner, E. C., J. Zheng, R. Zhang, W. B. Knighton, R. M. Volkamer, et al. (2009), Measurements of Volatile Organic Compounds Using Proton Transfer Reaction - Mass Spectrometry during the MILAGRO 2006 Campaign, Atmos. Chem. Phys., 9(2), 467-481.

Fuller, E. N., Schettle.Pd, and J. C. Giddings (1966), A New Method for Prediction of Binary Gas-Phase Diffusion Coeffecients, Ind. Eng. Chem., 58(5), 18-27.

Gao, X., and J. Chorover (2010), Adsorption of sodium dodecyl sulfate (SDS) at ZnSe and [a]-Fe2O3 surfaces: Combining infrared spectroscopy and batch uptake studies, J. Colloid Interf. Sci., 348(1), 167-176.

Gilardoni, S., L. M. Russell, A. Sorooshian, R. C. Flagan, J. H. Seinfeld, et al. (2007), Regional variation of organic functional groups in aerosol particles on four U.S. east coast platforms during the International Consortium for Atmospheric Research on Transport and Transformation 2004 campaign, J. Geophys. Res., 112(D10), D10S27.

Green, D. W., and R. H. Perry (2008), Perry's Chemical Engineers' Handbook (8th Edition), edited, McGraw-Hill, 2-82-2-86.

Guenther, A., T. Karl, P. Harley, C. Wiedinmyer, P. I. Palmer, et al. (2006), Estimates of global terrestrial isoprene emissions using MEGAN (Model of Emissions of Gases and Aerosols from Nature), Atmos. Chem. Phys., 6, 3181-3210.

Guenther, A., C. N. Hewitt, D. Erickson, R. Fall, C. Geron, et al. (1995), A Global-Model of Natural Volatile Organic-Compound Emissions, J. Geophys. Res-Atmos., 100(D5), 88738892.

Hallquist, M., J. C. Wenger, U. Baltensperger, Y. Rudich, D. Simpson, et al. (2009), The formation, properties and impact of secondary organic aerosol: current and emerging issues, Atmos. Chem. Phys., 9(14), 5155-5236.

Hatch, L. E., J. M. Creamean, A. P. Ault, J. D. Surratt, M. N. Chan, et al. (2011), Measurements of Isoprene-Derived Organosulfates in Ambient Aerosols by Aerosol Timeof-Flight Mass Spectrometry - Part 1: Single Particle Atmospheric Observations in Atlanta, Environ. Sci. Technol.

Iinuma, Y., O. Boge, A. Kahnt, and H. Herrmann (2009), Laboratory chamber studies on the formation of organosulfates from reactive uptake of monoterpene oxides, Phys. Chem. Chem. Phys., 11(36), 7985-7997. 
Iinuma, Y., C. Müller, T. Berndt, O. Böge, M. Claeys, et al. (2007), Evidence for the Existence of Organosulfates from $\beta$-Pinene Ozonolysis in Ambient Secondary Organic Aerosol, Environ. Sci. Technol., 41(19), 6678-6683.

Jang, M., N. M. Czoschke, S. Lee, and R. M. Kamens (2002), Heterogeneous Atmospheric Aerosol Production by Acid-Catalyzed Particle-Phase Reactions, Science, 298(5594), 814817.

Kanakidou, M., J. H. Seinfeld, S. N. Pandis, I. Barnes, F. J. Dentener, et al. (2005), Organic aerosol and global climate modelling: a review, Atmos. Chem. Phys., 5, 1053-1123.

Katritzky, A. R., S. Sild, and M. Karelson (1998), General Quantitative Structure-Property Relationship Treatment of the Refractive Index of Organic Compounds, J. Chem. Inf. Comp. Sci., 38(5), 840-844.

Kroll, J. H., and J. H. Seinfeld (2008), Chemistry of secondary organic aerosol: Formation and evolution of low-volatility organics in the atmosphere, Atmos. Environ., 42(16), 35933624.

Levitt, N. P., J. Zhao, and R. Zhang (2006), Heterogeneous chemistry of butanol and decanol with sulfuric acid: implications for secondary organic aerosol formation, J. Phys. Chem. A, 110(49), 13215-13220.

Ley, J. B., and C. A. Vernon (1957), 582. Reactions related to the pinacol-pinacone rearrangement. Part I. The acid-catalysed rearrangement of 2-methylpropane-1 : 2-diol and its ethers, J. Chem. Soc. (Resumed), 2987-2993.

Limbach, H. H. (1991), Dynamic NMR Spectroscopy in the Presence of Kinetic Hydrogen/Deuterium Isotope Effects, in NMR-Basic Principles and Progress, edited by P. Diehl, Fluck, E., pp. 63-164, Springer Heidelberg.

Maleknia, S. D., T. L. Bell, and M. A. Adams (2007), PTR-MS analysis of reference and plant-emitted volatile organic compounds, Int. J. Mass Spectrom., 262(3), 203-210.

McDonald, R. N., and A. K. Chowdhury (1985), Gas-phase ion-molecule reactions of dioxygen anion radical (O2-.bul.), J. Am. Chem. Soc., 107(14), 4123-4128.

Metzger, A., B. Verheggen, J. Dommen, J. Duplissy, A. S. H. Prevot, et al. (2010), Evidence for the role of organics in aerosol particle formation under atmospheric conditions, P. Natl Acad Sci., 107(15), 6646-6651.

Minerath, E. C., and M. J. Elrod (2009), Assessing the Potential for Diol and Hydroxy Sulfate Ester Formation from the Reaction of Epoxides in Tropospheric Aerosols, Environ. Sci. Technol., 43(5), 1386-1392. 
Minerath, E. C., M. T. Casale, and M. J. Elrod (2008), Kinetics feasibility study of alcohol sulfate esterification reactions in tropospheric aerosols, Environ. Sci. Technol., 42(12), 44104415.

Minerath, E. C., M. P. Schultz, and M. J. Elrod (2009), Kinetics of the Reactions of IsopreneDerived Epoxides in Model Tropospheric Aerosol Solutions, Environ. Sci. Technol., 43(21), 8133-8139.

Norman, M., A. Hansel, and A. Wisthaler (2007), $\mathrm{O}_{2}{ }^{+}$as reagent ion in the PTR-MS instrument: Detection of gas-phase ammonia, Int. J. Mass Spectrom., 265(2-3), 382-387.

Parker, R. E., and N. S. Isaacs (1959), Mechanisms Of Epoxide Reactions, Chem. Rev., 59(4), 737-799.

Paulot, F., J. D. Crounse, H. G. Kjaergaard, A. Kurten, J. M. St Clair, et al. (2009), Unexpected Epoxide Formation in the Gas-Phase Photooxidation of Isoprene, Science, 325(5941), 730-733.

Qiu, C., L. Wang, V. Lal, A. F. Khalizov, and R. Zhang (2011), Heterogeneous Reactions of Alkylamines with Ammonium Sulfate and Ammonium Bisulfate, Environ. Sci. Technol., 45(11), 4748-4755.

Schoon, N., C. Amelynck, L. Vereecken, and E. Arijs (2003), A selected ion flow tube study of the reactions of $\mathrm{H}_{3} \mathrm{O}^{+}, \mathrm{NO}^{+}$and $\mathrm{O}_{2}^{+}$with a series of monoterpenes, Int. J. Mass Spectrom., 229(3), 231-240.

Schoon, N., C. Amelynck, L. Vereecken, H. Coeckelberghs, and E. Arijs (2004), A selected ion flow tube study of the reactions of $\mathrm{H}_{3} \mathrm{O}^{+}, \mathrm{NO}^{+}$and $\mathrm{O}_{2}{ }^{+}$with some monoterpene oxidation products, Int. J. Mass Spectrom., 239(1), 7-16.

Schuttlefield, J. D., and V. H. Grassian (2008), ATR-FTIR Spectroscopy in the Undergraduate Chemistry Laboratory. Part I: Fundamentals and Examples, J. Chem. Educ., $85(2), 279$.

Seinfeld, J. H., and S. N. Pandis (2006), Atmospheric Chemistry and Physics - From Air Pollution to Climate Change (2nd Edition), edited, John Wiley \& Sons.

Skalny, J. D., T. Mikoviny, S. Matejcik, and N. J. Mason (2004), An analysis of mass spectrometric study of negative ions extracted from negative corona discharge in air, Int. J. Mass Spectrom., 233(1-3), 317-324.

Skov, H., T. Benter, R. N. Schindler, J. Hjorth, and G. Restelli (1994), Epoxide Formation in the Reactions of the Nitrate Radical with 2,3-Dimethyl-2-Butene, Cis-2-Butene and Trans-2Butene and Isoprene, Atmos. Environ., 28(9), 1583-1592. 
Socrates, G. (2004), Infrared and Raman characteristic group frequencies: tables and charts, John Wiley and Sons, Ltd., Chichester, $3^{\text {rd }}$ edition.

Surratt, J. D., M. Lewandowski, J. H. Offenberg, M. Jaoui, T. E. Kleindienst, et al. (2007), Effect of Acidity on Secondary Organic Aerosol Formation from Isoprene, Environ. Sci. Technol., 41(15), 5363-5369.

Surratt, J. D., A. W. H. Chan, N. C. Eddingsaas, M. Chan, C. L. Loza, et al. (2010), Reactive intermediates revealed in secondary organic aerosol formation from isoprene, P. Natl. Acad. Sci., 107(15), 6640-6645.

Surratt, J. D., S. M. Murphy, J. H. Kroll, N. L. Ng, L. Hildebrandt, et al. (2006), Chemical Composition of Secondary Organic Aerosol Formed from the Photooxidation of Isoprene, $J$. Phys. Chem. A, 110(31), 9665-9690.

Surratt, J. D., Y. Gómez-González, A. W. H. Chan, R. Vermeylen, M. Shahgholi, et al. (2008), Organosulfate Formation in Biogenic Secondary Organic Aerosol, J. Phys. Chem. A, 112(36), 8345-8378.

Tani, A., S. Hayward, and C. N. Hewitt (2003), Measurement of monoterpenes and related compounds by proton transfer reaction-mass spectrometry (PTR-MS), Int. J. Mass Spectrom., 223-224, 561-578.

Tani, A., S. Hayward, A. Hansel, and C. N. Hewitt (2004), Effect of water vapour pressure on monoterpene measurements using proton transfer reaction-mass spectrometry (PTR-MS), Int. J. Mass Spectrom., 239(2-3), 161-169.

Thibblin, A. (1988), Reaction branching as a mechanistic alternative to tunneling in explaining anomalous temperature-dependencies of kinetic isotope effects, J. Phys. Org. Chem., 1(3), 161-167.

Thibblin, A., and P. Ahlberg (1989), Reaction branching and extreme kinetic isotope effects in the study of reaction mechanisms, Chem. Soc. Rev., 18, 209-224.

Van Doorslaer, E., O. Van Opstal, H. Kersters-Hilderson, and C. K. De Bruyne (1984), Kinetic [alpha]-deuterium isotope effects for enzymatic and acid hydrolysis of aryl-[beta]-glycopyranosides, Bioorg. Chem., 12(2), 158-169.

Viidanoja, J., T. Reiner, and F. Arnold (1998), Laboratory investigations of negative ion molecule reactions of formic and acetic acids: implications for atmospheric measurements by ion-molecule reaction mass spectrometry, Int. J. Mass Spectrom., 181, 31-41.

Wang, L., W. Xu, A. F. Khalizov, J. Zheng, C. Qiu, et al. (2011), Laboratory Investigation on the Role of Organics in Atmospheric Nanoparticle Growth, J. Phys. Chem. A, 115(32), 8940-8947. 
Wang, L., V. Lal, A. F. Khalizov, and R. Zhang (2010), Heterogeneous chemistry of alkylamines with sulfuric acid: implications for atmospheric formation of alkylaminium sulfates, Environ. Sci. Technol., 44(7), 2461-2465.

Wang, L., A. F. Khalizov, J. Zheng, W. Xu, Y. Ma, et al. (2010), Atmospheric nanoparticles formed from heterogeneous reactions of organics, Nat. Geosci., 3(4), 238-242.

Warneke, C., J. A. de Gouw, W. C. Kuster, P. D. Goldan, and R. Fall (2003), Validation of Atmospheric VOC Measurements by Proton-Transfer- Reaction Mass Spectrometry Using a Gas-Chromatographic Preseparation Method, Environ. Sci. Technol., 37(11), 2494-2501.

Wiberg, K. B. (1955), The Deuterium Isotope Effect, Chem. Rev., 55(4), 713-743.

Yu, Y., M. J. Ezell, A. Zelenyuk, D. Imre, L. Alexander, et al. (2008), Photooxidation of [alpha]-pinene at high relative humidity in the presence of increasing concentrations of NOx, Atmos. Environ., 42(20), 5044-5060.

Zhang, D., and R. Zhang (2005), Laboratory investigation of heterogeneous interaction of sulfuric acid with soot, Environ. Sci. Technol., 39(15), 5722-5728.

Zhang, Q., J. L. Jimenez, D. R. Worsnop, and M. Canagaratna (2007), A Case Study of Urban Particle Acidity and Its Influence on Secondary Organic Aerosol, Environ. Sci. Technol., 41(9), 3213-3219.

Zhang, R., P. J. Wooldridge, J. P. D. Abbatt, and M. J. Molina (1993), Physical-Chemistry of the $\mathrm{H}_{2} \mathrm{SO}_{4} / \mathrm{H}_{2} \mathrm{O}$ Binary-System at Low-Temperatures - Stratospheric Implications, J. Phys. Chem-Us, 97(28), 7351-7358.

Zhang, R., I. Suh, J. Zhao, D. Zhang, E. C. Fortner, et al. (2004), Atmospheric New Particle Formation Enhanced by Organic Acids, Science, 304(5676), 1487-1490.

Zhao, J., N. P. Levitt, R. Zhang, and J. Chen (2006), Heterogeneous reactions of methylglyoxal in acidic media: implications for secondary organic aerosol formation, Environ. Sci. Technol., 40(24), 7682-7687.

Zhou, S., I. Barnes, T. Zhu, I. Bejan, and T. Benter (2006), Kinetic Study of the Gas-Phase Reactions of $\mathrm{OH}$ and $\mathrm{NO}_{3}$ Radicals and $\mathrm{O}_{3}$ with Selected Vinyl Ethers, J. Phys. Chem. A, 110(23), 7386-7392. 


\section{APPENDIX}

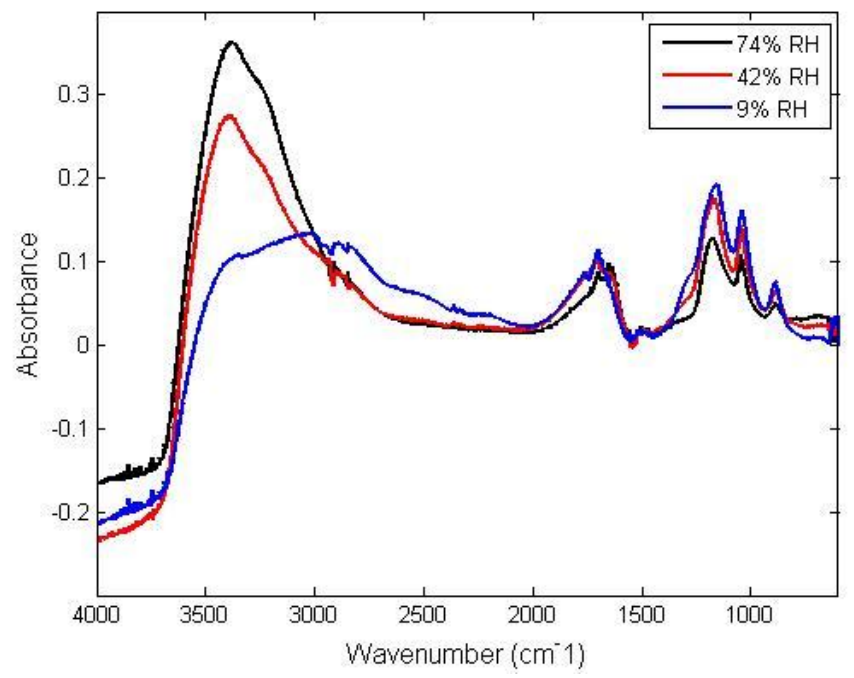

Figure 22: ATR-FTIR spectra of sulfuric acid at different RH.

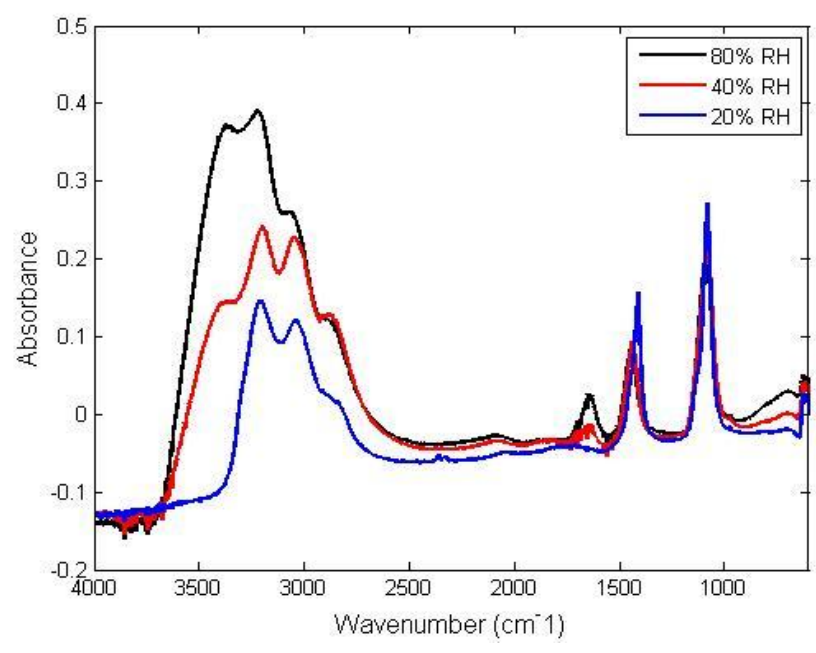

(a)

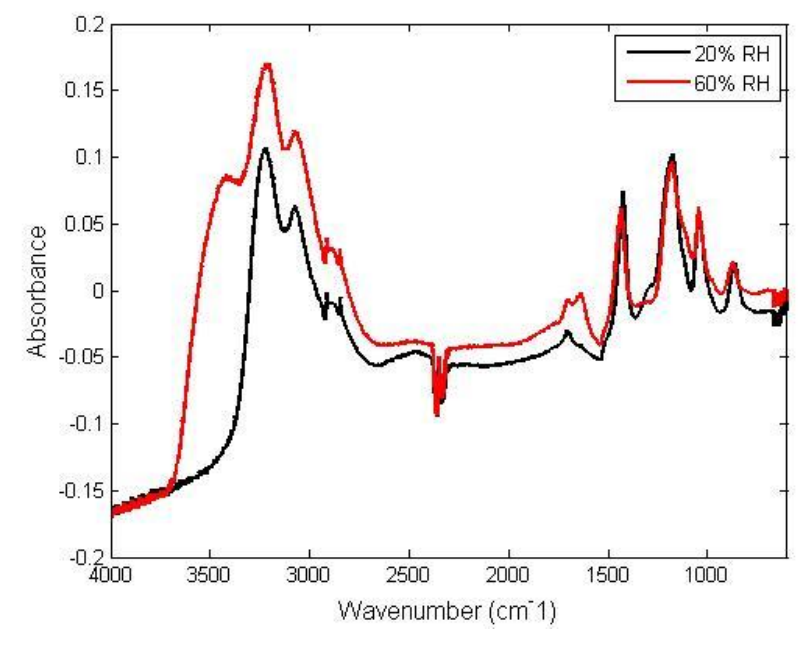

(b)

Figure 23: ATR-FTIR spectra at different RH for (a) Ammonium sulfate (b) Ammonium bisulfate. 


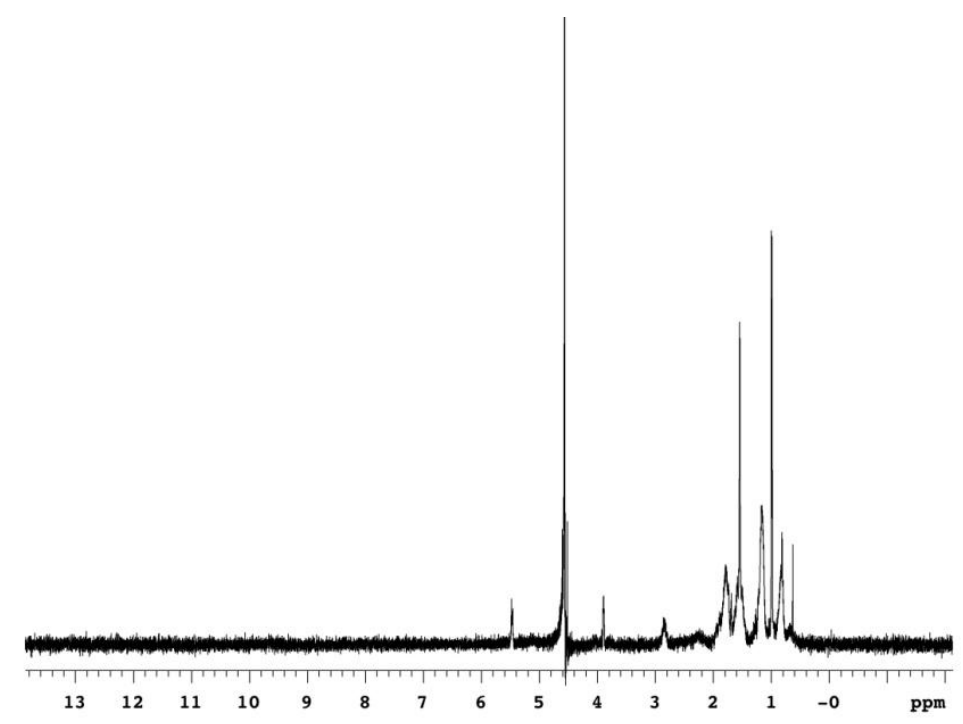

Figure 24: ${ }^{1} \mathrm{H}$ NMR spectrum of $\alpha$-pinene oxide reaction with $0.1 \%$ wt $\mathrm{D}_{2} \mathrm{SO}_{4} / 5 \%$ wt $\mathrm{Na}_{2} \mathrm{SO}_{4}$ solution.

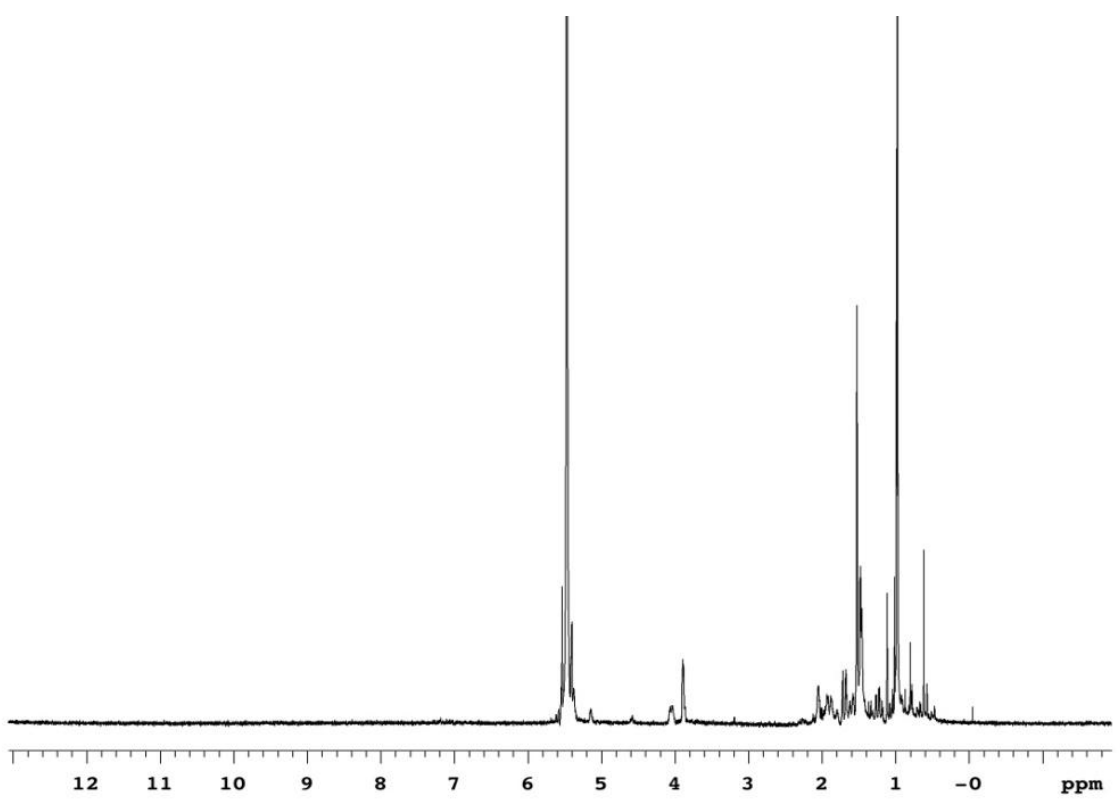

Figure 25: ${ }^{1} \mathrm{H}$ NMR spectrum of $\alpha$-pinene oxide reaction with $5 \%$ wt $\mathrm{D}_{2} \mathrm{SO}_{4}$ solution. 


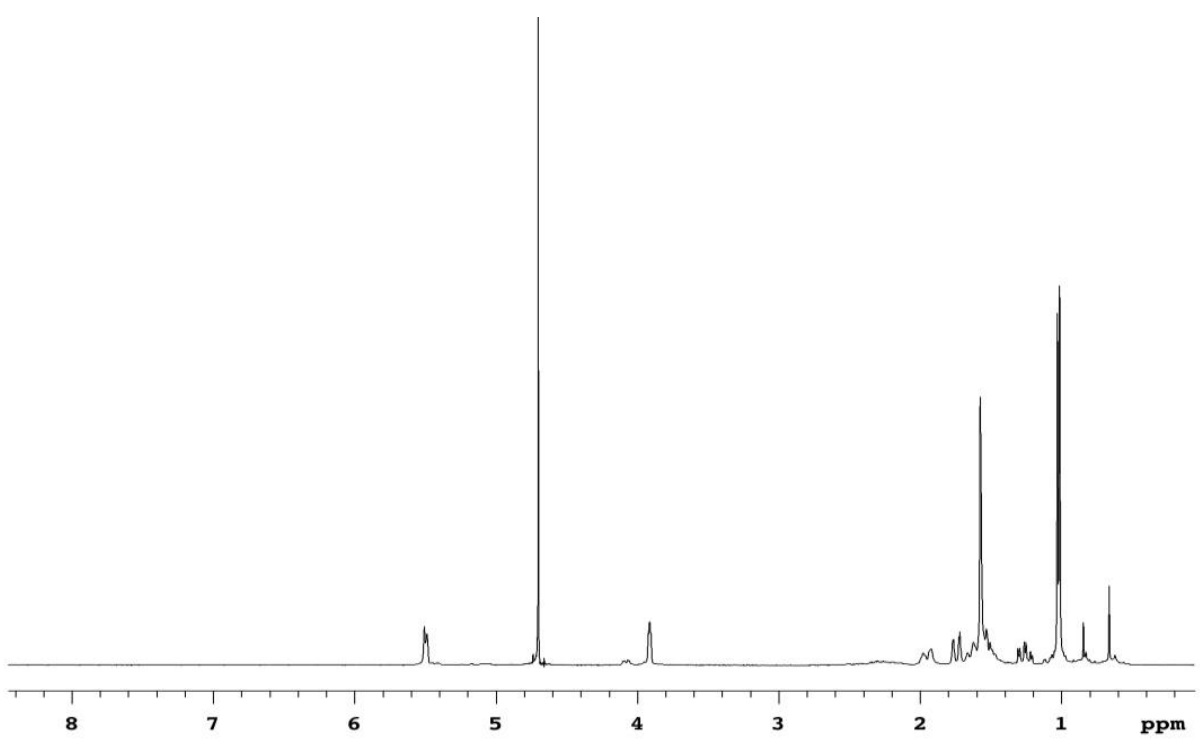

Figure 26: ${ }^{1} \mathrm{H}$ NMR spectrum of $\alpha$-pinene oxide reaction with $0.02 \%$ wt $\mathrm{D}_{2} \mathrm{SO}_{4}$ solution.

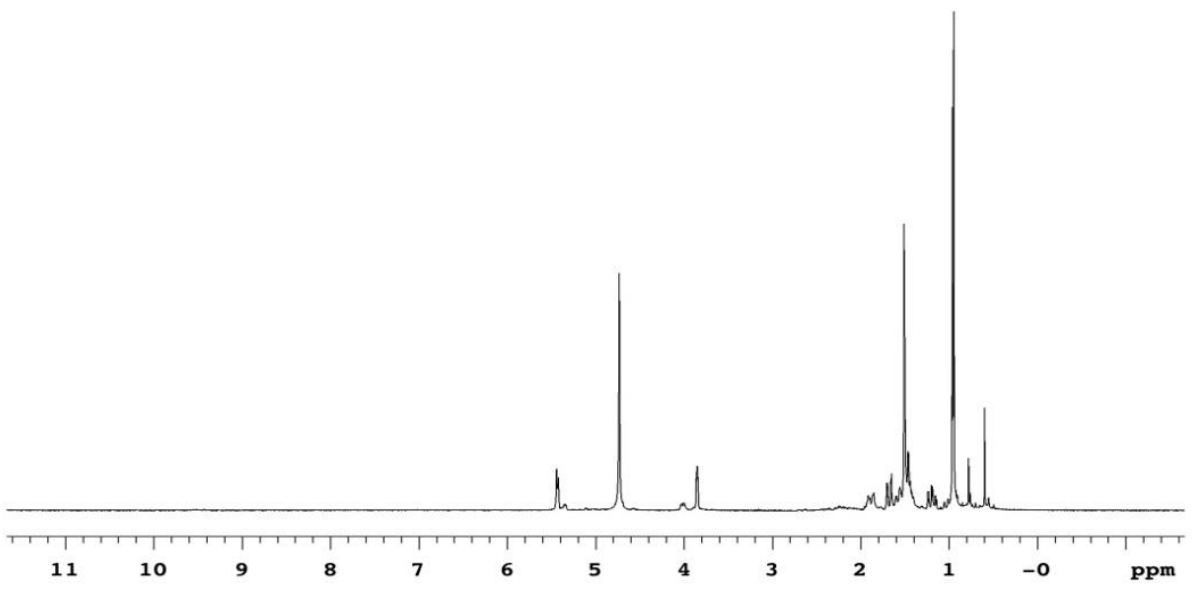

Figure 27: ${ }^{1} \mathrm{H}$ NMR spectrum of $\alpha$-pinene oxide reaction with $0.3 \%$ wt $\mathrm{D}_{2} \mathrm{SO}_{4}$ solution. 


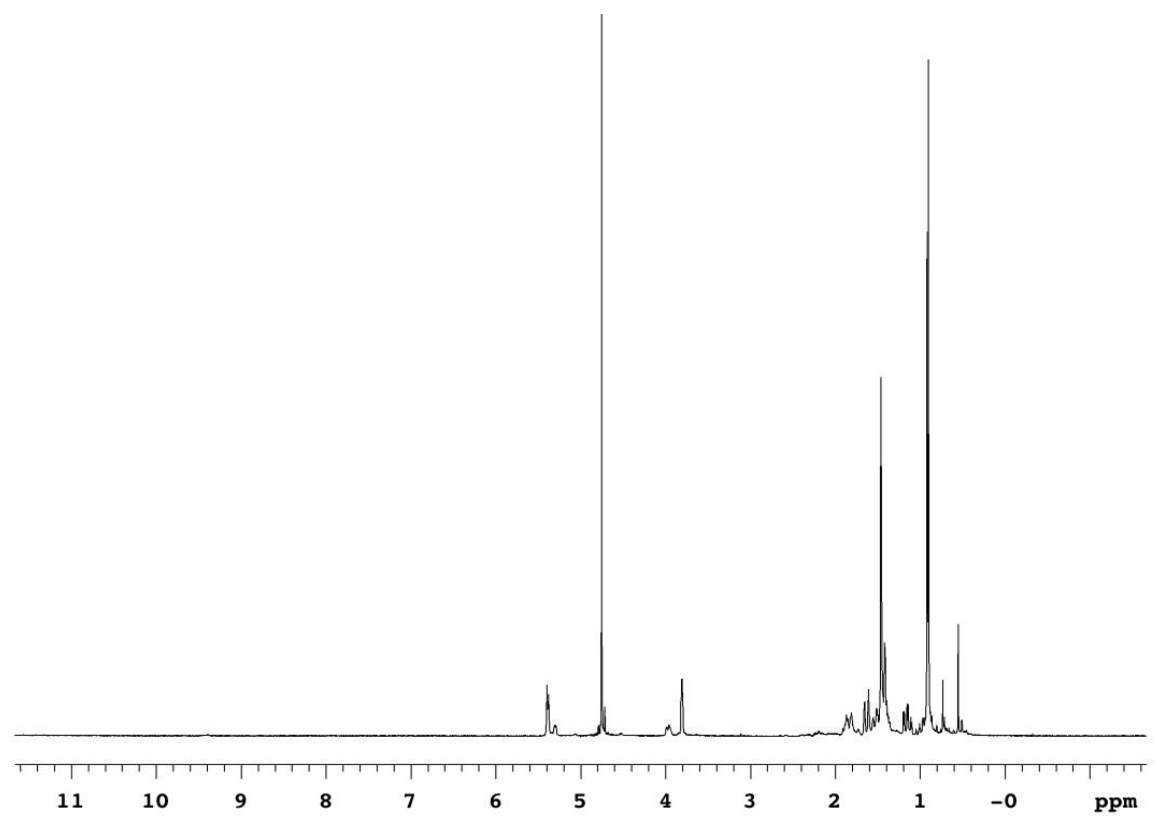

Figure 28: ${ }^{1} \mathrm{H}$ NMR spectrum of $\alpha$-pinene oxide reaction with $0.7 \%$ wt $\mathrm{D}_{2} \mathrm{SO}_{4}$ solution.

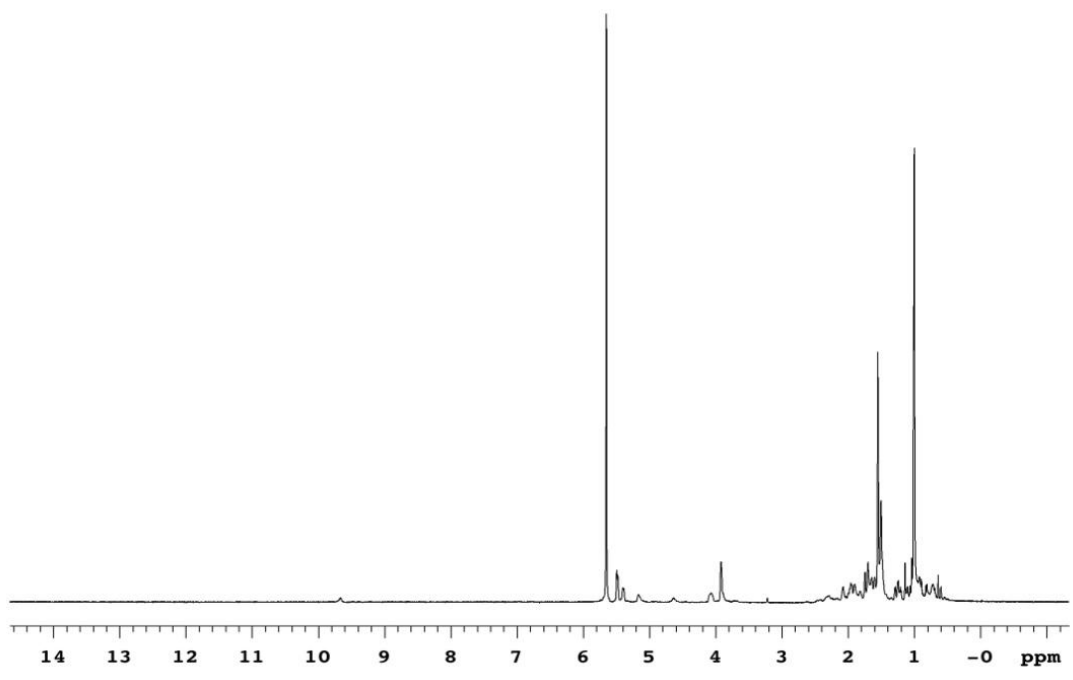

Figure 29: ${ }^{1} \mathrm{H}$ NMR spectrum of $\alpha$-pinene oxide reaction with $5 \% \mathrm{D}_{2} \mathrm{SO}_{4}$ with a higher epoxide concentration (acid: epoxide ratio 1:10). 


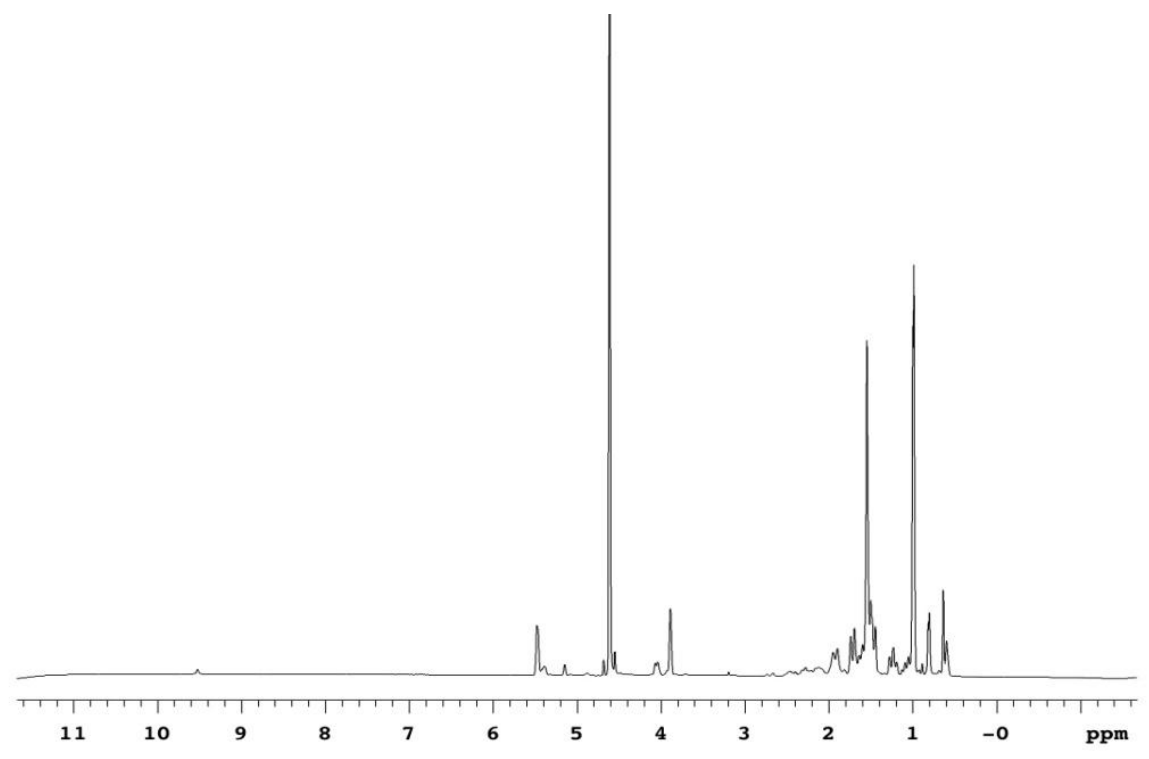

Figure 30: ${ }^{1} \mathrm{H}$ NMR spectrum of overnight experiment of $\alpha$-pinene oxide reaction with $5 \%$ $\mathrm{D}_{2} \mathrm{SO}_{4}$.

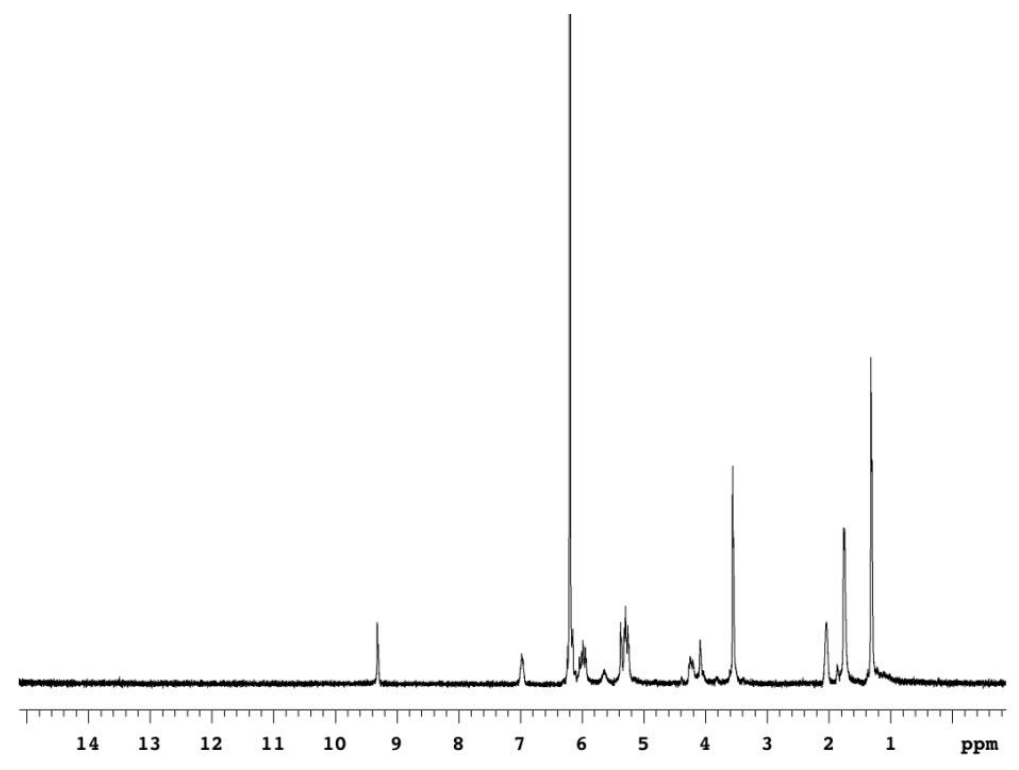

Figure 31: ${ }^{1} \mathrm{H}$ NMR spectrum of isoprene oxide reaction with $5 \%$ wt $\mathrm{D}_{2} \mathrm{SO}_{4}$ solution. 


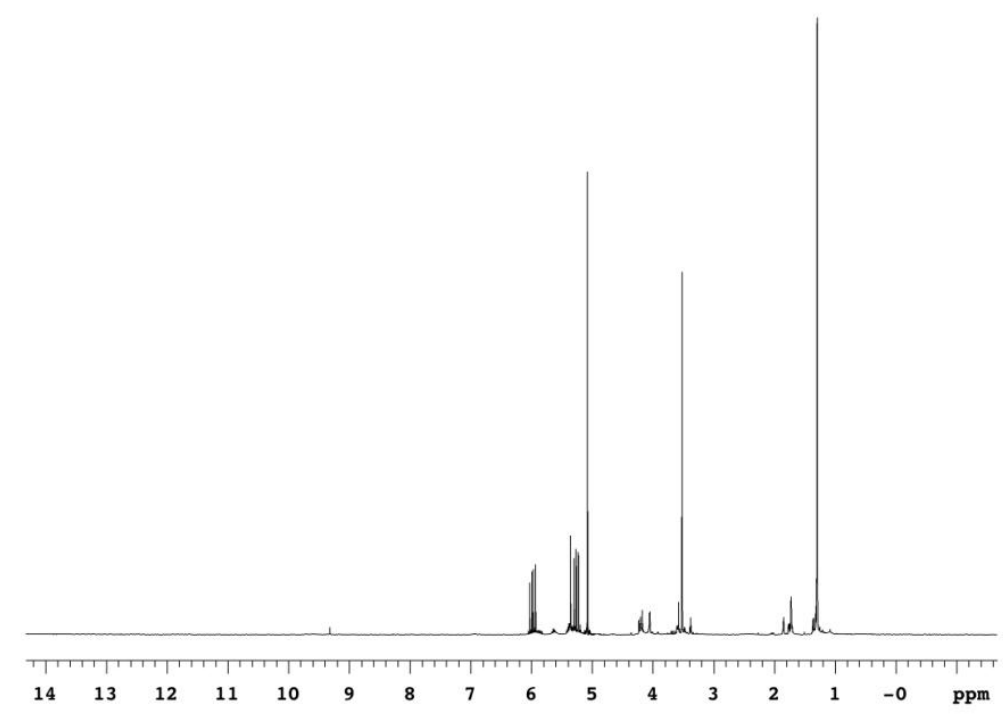

Figure 32: ${ }^{1} \mathrm{H}$ NMR spectrum of isoprene oxide reaction with $0.7 \%$ wt $\mathrm{D}_{2} \mathrm{SO}_{4}$ solution.

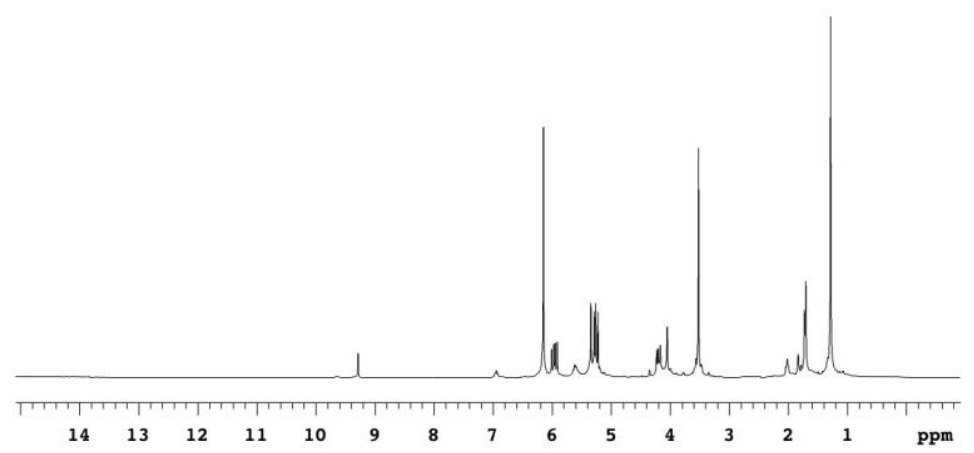

Figure 33: ${ }^{1} \mathrm{H}$ NMR spectrum of isoprene oxide reaction with $5 \%$ wt $\mathrm{D}_{2} \mathrm{SO}_{4}$ solution with higher epoxide concentration (acid: epoxide ratio 1:10). 


\section{VITA}

Name: $\quad$ Vinita Lal

Address: Department of Atmospheric Sciences, Texas A\&M University, College Station, Texas 77843-3150

Email: _dreamvins@gmail.com

Education: $\quad$ B.Sc., Chemistry, Banaras Hindu University, India, 2006

M.Sc., Environmental Science, Banaras Hindu University, India, 2008

M.S., Atmospheric Sciences, Texas A\&M University, 2011 